\begin{abstract}
Title of dissertation: MANY-BODY EFFECTS IN GRAPHENE

Wang-Kong Tse, Doctor of Philosophy, 2008

Dissertation directed by: Professor Sankar Das Sarma Department of Physics
\end{abstract}

Graphene is a novel material that features a quasi-relativistic linear energy dispersion with the quantum mechanical motion of electrons obeying the massless Dirac equation. In this dissertation, we study the many-body effects in graphene due to Coulomb interaction and electron-phonon interaction. Interaction effects can appear in both transport and electronic properties. For many-body effects in transport, we formulate the theory for Coulomb drag in double-layer graphene. We calculate the drag resistivity and study its dependence on temperature, density and interlayer spacing, finding zero drag if one of the graphene layers is intrinsic (i.e., undoped) and a non-zero drag exhibiting a similar behavior to regular bilayer drag if both graphene layers are extrinsic (i.e., doped). For many-body effects in electronic properties, we formulate the theory for quasiparticle and phonon renormalization due to Coulomb and electron-phonon interaction. We first study renormalization of electron properties due to Coulomb interaction by calculating the renormalized quasiparticle parameters from the electron self-energy, showing that 
intrinsic graphene behaves as a marginal Fermi liquid and extrinsic graphene behaves as a regular Fermi liquid. We then study renormalization of electron properties due to electron-phonon interaction. We calculate the electron self-energy and the renormalized quasiparticle velocity, finding that the renormalized band structure exhibits a kink at the phonon energy in agreement with angle-resolved photoemission spectroscopy (ARPES) experiment. We finally study renormalization of phonon energy due to electron-phonon interaction. We calculate the phonon self-energy and the renormalized phonon energy dispersion, showing that multiple Kohn anomalies arise which are completely different from the Kohn anomaly in usual metals. 


\title{
MANY-BODY EFFECTS IN GRAPHENE
}

\author{
by \\ Wang-Kong Tse \\ Dissertation submitted to the Faculty of the Graduate School of the \\ University of Maryland, College Park in partial fulfillment \\ of the requirements for the degree of \\ Doctor of Philosophy \\ 2008
}

Advisory Committee:

Professor Sankar Das Sarma, Chair/Advisor

Professor Theodore Einstein

Professor Michael Fuhrer

Professor Victor Galitski

Professor Ellen Williams 
(c) Copyright by Wang-Kong Tse 2008 
To my parents,

who have offered me their unconditional support through these years. 


\section{Acknowledgments}

I have heartfelt gratitude for all the people who have helped and supported me during these five years of my Ph.D. study. First of all, I would like to thank my advisor Professor Sankar Das Sarma for his insightful advice and helpful guidance, and for his motivation which inspired me on several occasions to get through the tough research problem at hand. It has been a true pleasure to work with and learn from him, who has created a wonderful environment for the Condensed Matter Theory Center (CMTC) where I have also benefited tremendously from interacting with other group members.

I remain very grateful to my family for being supportive of my educational and career goal. I am particularly grateful for for my mother who has supported me a great deal during these years and my father for instilling a learning environment at home when I was young. Without the support of my family, I would not have been able to pursue my goal and obtain my Ph.D. today. Words cannot express my gratitude to my family.

I would also like to express my gratitude to all the colleagues and friends who have helped and supported me in Maryland. I would like to thank Ben $\mathrm{Hu}$ from whom I learned a lot through enlightening and stimulating discussions (occasionally over dim sums at lunch); Euyheon Hwang for his patient and useful guidance in tackling difficult and detailed calculation problems; Igor Zutic for his useful advice to my career which has helped me a lot when I was starting out my research at CMTC and when I was preparing for my job hunting. 
Last but not least, I owe my gratitude to three great friends Stephen Ho, Don Priour and Wayne Witzel, who have provided their help and encouragements when I needed it, and who have enriched my life in Maryland through countless occasions of sharing stimulating discussions and interesting adventures. 


\section{Contents}

1 Graphene 1

1.1 Introduction . . . . . . . . . . . . . . . . . . . . 1

1.1.1 Graphene structure . . . . . . . . . . . . . . 2

1.1.2 Energy dispersion . . . . . . . . . . . . . . . 4

1.1.3 Important graphene parameters . . . . . . . . . . . . . . . . . 9

1.2 Angle-resolved photoemission spectroscopy

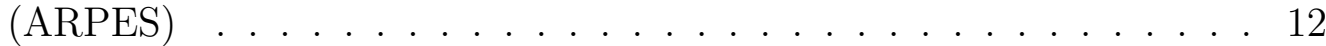

1.2.1 Graphene ARPES experiment . . . . . . . . . . . . 15

1.3 Outline of the thesis . . . . . . . . . . . . . . . 17

2 Quasiparticle renormalization due to Coulomb interaction 21

2.1 Random-phase approximation . . . . . . . . . . . . . . . . . 22

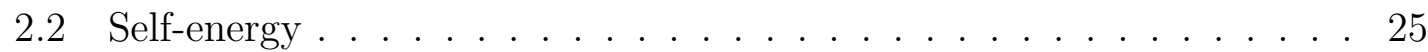

2.3 Extrinsic graphene . . . . . . . . . . . . . . . . . . . 28

2.3.1 Quasiparticle decay rate . . . . . . . . . . . . . 29

2.3.2 Renormalization factor . . . . . . . . . . . . . . 32

2.3.3 Renormalized velocity . . . . . . . . . . . . . 35

2.4 Intrinsic graphene . . . . . . . . . . . . . . . . . . . . 37

2.4.1 Quasiparticle decay rate . . . . . . . . . . . . 37

2.4.2 Renormalization factor . . . . . . . . . . . . . . . 39

2.4.3 Renormalized velocity ................ 40

3 Many-body effect in graphene transport - Coulomb drag 42

3.1 The phenomenon of Coulomb drag . . . . . . . . . . . . . . . . 42

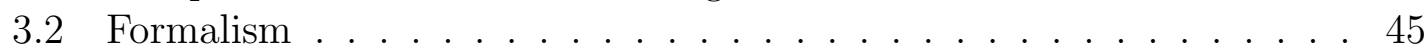

3.2.1 Drag conductivity . . . . . . . . . . . . . . 45

3.2.2 Nonlinear susceptibility . . . . . . . . . . . . . . . . 48

3.3 Drag in intrinsic graphene systems . . . . . . . . . . . . . . . 49

3.4 Drag in extrinsic graphene systems . . . . . . . . . . . . . . 51

3.4.1 Analytical results . . . . . . . . . . . . . . . . 51

3.4 .2 Numerical results . . . . . . . . . . . . . . . . . 58

3.5 Effect of interlayer plasmon excitation . . . . . . . . . . 60 
4 Quasiparticle renormalization due to electron-phonon interaction 64

4.1 Electron-phonon interaction . . . . . . . . . . . . . . . 64

4.2 Phonon-mediated electron-electron interaction . . . . . . . . . 68

4.3 Self-energy . . . . . . . . . . . . . . . . . . . . . . 71

4.3.1 Imaginary part of the self-energy _ . . . . . . . . . . 73

4.3.2 Real part of the self-energy _. . . . . . . . . . . . 75

4.3 .3 Quasiparticle spectral function . . . . . . . . . . . . . . . 80

4.3.4 Renormalized energy dispersion . . . . . . . . . . . . 81

5 Phonon renormalization due to electron-phonon interaction 85

5.1 Validity of the Born-Oppenheimer

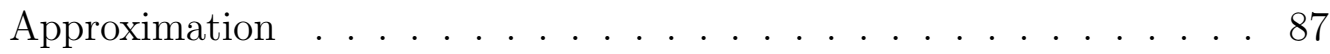

5.2 Formalism . . . . . . . . . . . . . . . . . . . . . . . . . . . . . 89

5.3 Phonon self-energy $\ldots \ldots \ldots \ldots \ldots$

5.3.1 Long-wavelength limit . . . . . . . . . . . . . . 96

5.3 .2 Static limit . . . . . . . . . . . . . . . 97

$5.3 .3 \quad$ Finite $q$ and $\omega \ldots \ldots \ldots \ldots$. . . . . . . . . . . 98

5.4 Renormalized phonon energy dispersion . . . . . . . . . . . . . 99

5.5 Understanding the Kohn anomalies . . . . . . . . . . . . . 101

6 Conclusion and Outlook 104

$\begin{array}{ll}\text { List of Publications } & 108\end{array}$

$\begin{array}{ll}\text { Bibliography } & 110\end{array}$ 


\section{List of Figures}

1.1 The real-space honeycomb lattice structure of graphene. A unit cell is formed by connecting the centers of four adjacent hexagonal lattices, and comprises two atoms labeled A and B. $\boldsymbol{a}_{1}$ and $\boldsymbol{a}_{2}$ are the lattice vectors, $\boldsymbol{e}_{1}, \boldsymbol{e}_{2}, \boldsymbol{e}_{3}$ are the unit vectors along the three possible directions of the hexagon. . . . . . . . . . . .

1.2 The reciprocal-space structure of graphene, which is also a honeycomb lattice. The Brillouin zone is indicated by the shaded hexagon, with corners at $\mathrm{K}$ and $\mathrm{K}$ ' points. $\boldsymbol{b}_{1}$ and $\boldsymbol{b}_{2}$ are the reciprocal space vectors. 4

1.3 The energy-momentum dispersion surface of graphene (adapted from Fig. 3 of Ref. [1]). The zoom-in figure depicts the energy dispersion in the vicinity of the K point, which exhibits a cone-like structure. . .

1.4 Geometry of an ARPES experiment. (adapted from Fig. 6(a) of Ref. [2]) The electron analyzer registers the photoemission intensity at a polar angle $\theta$ and azithmuthal angle $\phi . \ldots \ldots \ldots 13$

1.5 ARPES intensity (a) EDCs, (b) MDCs and (c) measured on the material Bi2212 (adapted from Ref. [3]). The EDCs are shown vertically displaced for different values of momentum and the MDCs for different values of energy. . . . . . . . . . . . . . 15

1.6 Constant energy maps adapted from Fig. 1c-d of Ref. [4], in which the Fermi level around the six K and K' points are clearly discernible. Fig. $1.6 \mathrm{c}$ corresponds to an electron doping with a Fermi level of $0.45 \mathrm{eV}$ above the Dirac point and Fig. 1d to a hole doping with a Fermi level of $1.5 \mathrm{eV}$ below the Dirac point. It can be seen that from Fig. $1.6 \mathrm{~d}$ that at such a large energy away from the Dirac point the energy dispersion is no longer linear. . . . . . . . . . . . 16 
1.7 Band structure of graphene at the Brillouin zone corner K adapted from Fig. 2 of Ref. [4]. The colored plots show the measured intensity, and the dashed lines indicate an extrapolation of the lower band. The number in each subfigure shows the electron density in $\mathrm{cm}^{-2}$. Fig. 1.7i shows the reconstructed spectral function using the extracted imaginary part of the self-energy from the line-width of MDCs. . . . . 17

1.8 Measured line-widths of the MDCs at different values of electron density $n$ (in units of $10^{13} \mathrm{~cm}^{-2}$ ) from Ref. [4]. . . . . . . . . . . . . . . 18

2.1 The Feynman diagram for the screened Coulomb interaction within the RPA. The thin and thick wiggly lines denote respectively the bare and the RPA-screened interaction, and the thin straight line stands for the bare Green function. . . . . . . . . . . . . . . . . . 23

2.2 The electron self-energy $\Sigma$ Eq. (2.15), the thick wiggly line denotes the screened Coulomb interaction within the RPA in Fig. 2.1. This is the standard ring diagram approximation for self-energy exact in the $r_{s} \ll 1$ limit

3.1 The experimental setting of a Coulomb drag experiment. A current is sent through the "active layer" driving electrons to flow along the layer, this "drags along" the electrons to move in the same direction in the open-circuited "passive layer" via interlayer Coulomb interaction, which then induce a voltage that can be measured. . . . . . . . . . . 43

3.2 Diagram for the nonlinear susceptibility $\Gamma$, which is a three-point vertex function. . . . . . . . . . . . . . . . . . .

3.3 Diagrams contributing to the drag resistivity Eq. (3.2). The double wavy lines represent the screened interlayer Coulomb potential Eq. (3.4) and the vertices on the left and on the right denote charge current in the two layers. Dashed vertical lines next to the vertices denote impurity vertex correction to the charge current. . . . . . . .

$3.4 \rho_{\mathrm{D}} /\left(T / T_{F}\right)^{2}$ as a function of $T / T_{F}$ for Coulomb drag between two identical extrinsic graphene sheets, with values of $k_{F} d=10$ (solid lines), 5 (dashed lines), and 1 (dot-dashed lines). Numerical results are indicated with bold (black) lines and analytical results Eq. (3.15) with thin (grey/red) lines. The analytical results become an increasingly accurate approximation to the full numerical results with increasing $k_{F} d$ (i.e. increasing $n$ or $d$ ). . . . . . . . . . . . . . . 60 
$3.5 \rho_{\mathrm{D}} /\left(T / T_{F}\right)^{2}$ vs. $T / T_{F}$ for higher values of $T$ up to $0.2 T_{F}$. Upper panel: for fixed interlayer distance $d=500 \AA$ and different values of density $n=10^{11} \mathrm{~cm}^{-2}$ (solid line), $5 \times 10^{11} \mathrm{~cm}^{-2}$ (dashed line), $10^{12} \mathrm{~cm}^{-2}$ (dot-dashed line), corresponding to $T_{F}=431 \mathrm{~K}, 963 \mathrm{~K}$, $1361 \mathrm{~K}$ respectively; lower panel: for fixed density $n=10^{11} \mathrm{~cm}^{-2}$ and different values of interlayer distance $d=300 \AA$ (solid line), $150 \AA$ (dashed line) and $30 \AA$ (dot-dashed line).

4.1 The G band in-plane optical phonon mode of graphene. The Asublattice atoms and B-sublattice atoms vibrate in opposite direction with each other. . . . . . . . . . . . . . . .

4.2 Raman spectrum obtained from experiment [5] for graphite and graphene. The peak at the wavenumber $1580 \mathrm{~cm}^{-1}$ is the $\mathrm{G}$ peak corresponding to the zone-center in-plane optical phonons; the band at $2700 \mathrm{~cm}^{-1}$ is the $\mathrm{G}^{\prime}$ band corresponding to a second order Raman scattering of the zone-boundary phonons. . . . . . . . . . . . . . 66

4.3 Feynman diagram for the phonon-mediated electron-electron interaction Eq. (4.10). The solid straight lines denote the electron Green function, and the zig-zag line denotes the phonon Green function. The electron-phonon interaction vertex is given by $g M_{\alpha^{\prime} \alpha}, g M_{\beta^{\prime} \beta}$ on the two vertices. An electron initially in the state $\left|\boldsymbol{k}_{1} \alpha\right\rangle$ interacts with another electron in the state $\left|\boldsymbol{k}_{2} \beta\right\rangle$ through exchanging a phonon of momentum $\boldsymbol{q}$, and then the first electron is scattered off to the state $\left|\boldsymbol{k}_{1}+\boldsymbol{q} \alpha^{\prime}\right\rangle$ while the second electron is scattered off to the state $\mid \boldsymbol{k}_{2}-$ $\left.\boldsymbol{q} \beta^{\prime}\right\rangle$. Since the electron-phonon interaction vertices $g M_{\alpha^{\prime} \alpha}, g M_{\beta^{\prime} \beta}$ are off-diagonal matrices, the pseudospin labels of the two electrons are flipped in the process: $\alpha^{\prime}=-\alpha$ and $\beta^{\prime}=-\beta \ldots \ldots \ldots$

4.4 The real and imaginary parts of the self-energy at $k=k_{F}$ for density $n=10^{13} \mathrm{~cm}^{-2}$. The red dashed line shows the position of the Dirac point. Region $\omega>\omega_{0}$ corresponds to the intraband term $F_{\text {intra }}\left(k, \omega, \omega_{0}\right)$ whereas $-\omega_{0}-\varepsilon_{F}<\omega<-\omega_{0}$ corresponds to the intraband term $F_{\text {intra }}\left(k, \omega,-\omega_{0}\right)$. The first dip in $|\operatorname{Im} \Sigma|$ on the left side of the Dirac point marks the onset of the interband transitions $F_{\text {inter }}$ for $\omega<-\omega_{0}-\varepsilon_{F}$; the second dip occurring at $-\omega_{0}-\epsilon_{k}-\varepsilon_{F}$ originates from the particular form of the angular factor in Eq. (4.14) which comes from the electron-phonon interaction vertex Eq. (4.11).

4.5 The spectral function $A_{k}(\omega)$ (normalized to dimensionless unit by multiplying $\left.\omega_{0}\right)$ as a function of $\omega / \omega_{0}$ and $k / k_{F}$ for density $n=$ $10^{13} \mathrm{~cm}^{-2}$, the inset shows the locus of the position of the quasiparticle delta function inside the gap $\omega / \omega_{0} \in[-1,1] \ldots \ldots \ldots$ 
4.6 The renormalized conduction band energy spectrum for $n=10^{13} \mathrm{~cm}^{-2}$. The colored intensity plot shows the magnitude of the spectral function $A_{k}(\omega)$ whereever it is non-zero, while the dashed white line shows the region within the amount of phonon energy $\omega_{0}=0.196 \mathrm{eV}$ from the Fermi level where $A_{k}(\omega)=0$. The thin dot-dashed straight line shows the bare unrenormalized spectrum. . . . . . . . . . . . . 82

4.7 The effective electron-phonon "coupling" parameter $\lambda_{\text {eff }}=v / v^{*}\left(k_{F}\right)-$ 1 as a function of doping $n . \ldots . \ldots . \ldots . \ldots 83$

5.1 (a) Dyson equation for the renormalization of the phonon Green function. The zigzag lines denote the phonon Green function and the crosses denote the electron-phonon interaction vertex. The shaded bubble with two cross vertices stands for the renormalized phonon self-energy. (b) Equation for the renormalized phonon self-energy. The unshaded bubble with two cross vertices denotes the bare phonon self-energy, the two bubbles with one cross vertex and one dot vertex are "hybrid bubbles" with one electron-phonon interaction vertex and one Coulomb interaction vertex. The wavy line stands for the usual RPA-screened Coulomb interaction. . . . . . . . . . . . . . . 90

5.2 Dynamic phonon self-energy $\tilde{\Pi}_{+}^{\mathrm{pp}}(x, u)$ versus $x=q / k_{F}$ at $u=\omega_{0} / \varepsilon_{F}$ for the LO (solid line) and TO (dashed) modes at a density $n=$ $10^{13} \mathrm{~cm}^{-2}$. Three cusps are apparent which correponds to the Kohn anomalies at $x=u, x=2 \pm u$ for LO phonons. For TO phonons, $\tilde{\Pi}_{+}^{\text {pp }}$ diverges at $x=u$. Inset: Static phonon self-energy $\tilde{\Pi}_{+}^{\mathrm{pp}}(x, u)$ versus $x=q / k_{F}$ at $u=0$. $\tilde{\Pi}_{+}^{\mathrm{pp}}(x, 0)$ for the LO mode has a Kohn anomaly at $q=2 k_{F}$. $\tilde{\Pi}_{+}^{\mathrm{pp}}(x, 0)$ for the TO mode is zero. . . . . . . . . . . 98

5.3 Static polarizability of graphene (adapted from Ref. [6]), with $\Pi_{\text {tot }}=$ $\Pi_{0}^{\mathrm{cc}}$ in the notation of this dissertation. $\Pi_{0}^{\mathrm{cc}}$ does not show any cusp or kink structure for finite values of $q \ldots$. . . . . . . . . . . . . . . 999

5.4 Renormalized LO phonon energy spectrum $\omega_{\text {ph }}$ versus $q$ at different electron densities $n$. The bare phonon energy is shown as the horizontal solid line. The range of phonon wavevector $q$ shown corresponds to $[0,0.08(2 \pi / a)]$ away from the $\Gamma$ point. . . . . . . . . . . . . . 100

5.5 Renormalized TO phonon energy spectrum $\omega_{\text {ph }}$ versus $q$. The legends are the same as in Fig. 5.4. . . . . . . . . . . . . . . . . . 101

5.6 Different regions for the analytical behavior of $\Pi_{+}^{\mathrm{pp}}$. The solid lines indicate the boundaries for these regions, and the dashed line shows the phonon energy $\omega=\omega_{0}$ at a density $n=10^{13} \mathrm{~cm}^{-2}$. . . . . . . . 102 


\section{Chapter 1}

\section{Graphene}

\section{$1.1 \quad$ Introduction}

Graphene is a two-dimensional hexagonal lattice of carbon atoms. It can be regarded as the fundamental building block of other important carbon-based structures, such as graphite (a stack of graphene sheets bonded by the van der Waals force) and carbon nanotubes (a cylinder formed by rolling up a graphene sheet). Graphene was first realized [7] experimentally by the group of Andre Geim at the University of Manchester in 2004, employing a surprisingly simple but effective method of ripping weakly bound layers from a graphite crystal using adhesive tape. Those layers are then rubbed against an oxidized silicon surface and put under an optical microscope to look for the rare monolayer flakes that would be graphene. Before its discovery, pure graphene was presumed not to exist in nature as early theoretical studies $[8,9,10,11]$ predicted that a two-dimensional crystal would be thermodynamically unstable and would spontaneously decompose, segregate or 
rearrange itself into other three-dimensional structures. This unexpected breakthrough therefore immediately caught the attention of the worldwide condensed matter physics community, followed by a burst of experimental and theoretical investigations resulting in more than six hundred papers in the four-year time-frame since its discovery. Several reviews in the subject that appeared in the literature are Refs. [12, 1, 13, 14].

One of the most interesting aspects of graphene that captures the interest of the condensed matter community is the linear energy dispersion of electrons at the Brillouin zone corners. This gives the electrons in the vicinity of those points the property of relativistic particles, obeying the massless Dirac equation as for massless neutrinos in relativistic quantum field theory. The band structure of graphene that gives rise to the peculiar linear dispersion at the Brillouin zone corners was first discussed by Wallace [15] in 1947 using tight-binding analysis, and then followed by McClure [16] and Slonczewski et al. [17]. These intitial theoretical studies of graphene served more as a stepping stone to understand the properties of graphite rather than as a subject of its own interest.

\subsubsection{Graphene structure}

The hexagonal real-space structure of graphene comprises two interpenetrating triangular sublattices A and B (Fig. 1.1), and this real-space structure also translates into a hexagonal lattice in momentum space. The Brillouin zone is a hexagon indicated by the shaded area in Fig. 1.2, with corners at the high symmetry $\mathrm{K}$ and 
K' points. When undoped, graphene has point Fermi surfaces located at these K and K' points, which are also called "valleys" by virtue of the fact that these are the troughs of the electron energy dispersion surface. The sublattices and valleys constitute additional electronic degrees of freedom besides the electron spin, with the A and B sublattices referred as the "pseudospins" and the $\mathrm{K}$ and $\mathrm{K}$ ' valleys referred as the "isospins". In the absence of any isospin symmetry-breaking process (i.e., intervalley impurity scattering, or Umklapp phonon scattering between a K valley point and a $\mathrm{K}^{\prime}$ valley point), the isospin degree of freedom is degenerate. In addition to the two electronic spins, this yields an combined electronic degeneracy factor of $2 \times 2=4$.

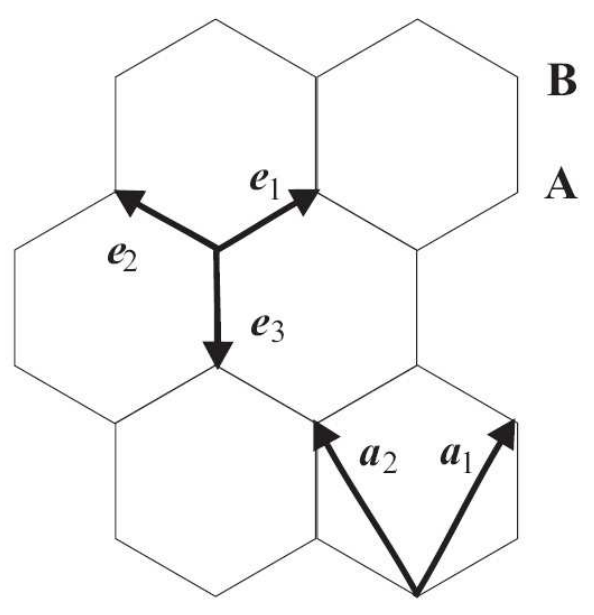

Figure 1.1: The real-space honeycomb lattice structure of graphene. A unit cell is formed by connecting the centers of four adjacent hexagonal lattices, and comprises two atoms labeled A and B. $\boldsymbol{a}_{1}$ and $\boldsymbol{a}_{2}$ are the lattice vectors, $\boldsymbol{e}_{1}, \boldsymbol{e}_{2}, \boldsymbol{e}_{3}$ are the unit vectors along the three possible directions of the hexagon. 


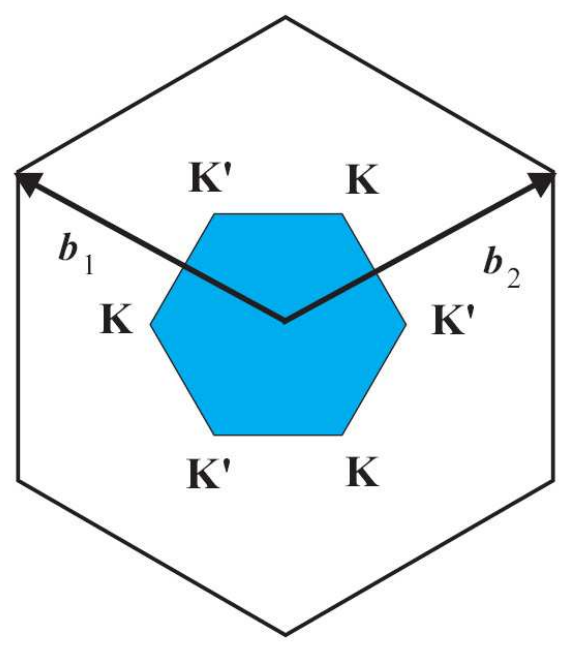

Figure 1.2: The reciprocal-space structure of graphene, which is also a honeycomb lattice. The Brillouin zone is indicated by the shaded hexagon, with corners at $\mathrm{K}$ and $\mathrm{K}^{\prime}$ points. $\boldsymbol{b}_{1}$ and $\boldsymbol{b}_{2}$ are the reciprocal space vectors.

\subsubsection{Energy dispersion}

In the following we derive the effective Hamiltonian in the neighborhood of the $\mathrm{K}$ point from the tight-binding model [15]. To this end, we define the vectors of the real-space lattice (Fig. 1.1)

$$
\begin{aligned}
& \boldsymbol{a}_{1}=\frac{\sqrt{3} a}{2}(1, \sqrt{3}) \\
& \boldsymbol{a}_{2}=\frac{\sqrt{3} a}{2}(-1, \sqrt{3})
\end{aligned}
$$

where $a=1.42 \AA$ is the distance between adjacent carbon atoms, and the reciprocal lattice vectors (Fig. 1.2)

$$
\begin{aligned}
& \boldsymbol{b}_{1}=\frac{2 \pi}{3 a}(\sqrt{3}, 1) \\
& \boldsymbol{b}_{2}=\frac{2 \pi}{3 a}(-\sqrt{3}, 1)
\end{aligned}
$$


and the three unit vectors connecting any atom to its nearest neighbors

$$
\begin{aligned}
& \boldsymbol{e}_{1}=\frac{a}{2}(\sqrt{3}, 1) \\
& \boldsymbol{e}_{2}=\frac{a}{2}(-\sqrt{3}, 1) \\
& \boldsymbol{e}_{3}=a(0,-1)
\end{aligned}
$$

The tight-binding Hamiltonian, in second quantized form, for the $\pi$-orbital electrons taking into account nearest-neighbor hopping is given by (next nearest-neighbor hopping can be neglected in the vicinity of the $\mathrm{K}$ point):

$$
H_{\mathrm{TB}}=-t \sum_{\langle i, j\rangle \sigma}\left(a_{i \sigma}^{\dagger} b_{j \sigma}+b_{j \sigma}^{\dagger} a_{i \sigma}\right),
$$

where $a_{i \sigma}, b_{i \sigma}$ are the electron annihilation operators for the sublattice A and $\mathrm{B}, i, j$ are lattice site labels (with the corresponding coordinate vectors $\boldsymbol{R}_{i}$ and $\boldsymbol{R}_{j},\langle i, j\rangle$ denotes the set of $i$ and $j$ in the nearest neighborhood of one another, $\sigma$ is the spin label, and $t \approx 2.7 \mathrm{eV}$ is the nearest-neighbor hopping energy. To express Eq. (1.4) in the momentum space, we define the Fourier transforms of $a_{i \sigma}$ and $b_{i \sigma}$

$$
\begin{aligned}
& a_{i \sigma}=\frac{1}{\sqrt{N}} \sum_{k} e^{-i \boldsymbol{k} \cdot \boldsymbol{R}_{i}} a_{k \sigma}, \\
& b_{i \sigma}=\frac{1}{\sqrt{N}} \sum_{k} e^{-i \boldsymbol{k} \cdot \boldsymbol{R}_{i}} b_{k \sigma},
\end{aligned}
$$

where $N$ is the number of unit cells. The tight-binding Hamilonian can now be expressed as

$$
\begin{aligned}
H_{\mathrm{TB}} & =\sum_{k \sigma}\left[\alpha(\boldsymbol{k}) a_{k \sigma}^{\dagger} b_{k \sigma}+\alpha^{*}(\boldsymbol{k}) b_{k \sigma}^{\dagger} a_{k \sigma}\right] \\
& =\sum_{k \sigma}\left[\begin{array}{ll}
a_{k \sigma}^{\dagger} & b_{k \sigma}^{\dagger}
\end{array}\right]\left[\begin{array}{cc}
0 & \alpha(\boldsymbol{k}) \\
\alpha^{*}(\boldsymbol{k}) & 0
\end{array}\right]\left[\begin{array}{l}
a_{k \sigma} \\
b_{k \sigma}
\end{array}\right] .
\end{aligned}
$$


where

$$
\alpha(\boldsymbol{k})=-t\left(1+e^{-i \boldsymbol{k} \cdot \boldsymbol{a}_{1}}+e^{-i \boldsymbol{k} \cdot \boldsymbol{a}_{2}}\right) .
$$

The energy dispersion is given by diagonalizing the Hamiltonian Eq. (1.6),

$$
\begin{aligned}
\epsilon_{ \pm} & = \pm|\alpha(\boldsymbol{k})| \\
& = \pm t \sqrt{3+2 \cos \left(\sqrt{3} k_{x} a\right)+4 \cos \left(\frac{\sqrt{3} k_{x} a}{2}\right) \cos \left(\frac{3 k_{y} a}{2}\right)} .
\end{aligned}
$$

The wave vector at the $\mathrm{K}$ and $\mathrm{K}^{\prime}$ points are $\boldsymbol{K}=\left(\boldsymbol{b}_{1}-\boldsymbol{b}_{2}\right) / 3=(4 \pi / 3 \sqrt{3} a)(1,0)$ and $\boldsymbol{K}^{\prime}=-\left(\boldsymbol{b}_{1}-\boldsymbol{b}_{2}\right) / 3=-(4 \pi / 3 \sqrt{3} a)(1,0)$. It can be easily verified that $\alpha(\boldsymbol{K})=$ $\alpha\left(\boldsymbol{K}^{\prime}\right)=0$. Expanding $\alpha(\boldsymbol{k})$ around $\boldsymbol{K}$ such that $\boldsymbol{k}=\boldsymbol{K}+\delta \boldsymbol{k}$,

$$
\begin{aligned}
\alpha(\boldsymbol{K}+\delta \boldsymbol{k})= & -t\left\{1+\exp \left\{-i\left[\frac{2 \pi}{3}+\frac{\sqrt{3} a}{2}\left(\delta k_{x}+\sqrt{3} \delta k_{y}\right)\right]\right\}\right. \\
& \left.+\exp \left\{-i\left[-\frac{2 \pi}{3}+\frac{\sqrt{3} a}{2}\left(-\delta k_{x}+\sqrt{3} \delta k_{y}\right)\right]\right\}\right\} \\
\simeq & \frac{3 t a}{2}\left(\delta k_{x}-i \delta k_{y}\right)
\end{aligned}
$$

Therefore the energy dispersion near the $\mathrm{K}$ point is given by

$$
\begin{aligned}
\epsilon_{ \pm} & = \pm \frac{3 t a}{2} \sqrt{\delta k_{x}^{2}+\delta k_{y}^{2}} \\
& = \pm v \delta k
\end{aligned}
$$

where $v=3 t a / 2$ is the Fermi velocity of the electron. The Hamiltonian Eq. (1.6) then becomes

$$
H_{\mathrm{TB}}=\sum_{k \sigma}\left[\begin{array}{ll}
a_{k \sigma}^{\dagger} & b_{k \sigma}^{\dagger}
\end{array}\right]\left[\begin{array}{cc}
0 & v\left(\delta k_{x}-i \delta k_{y}\right) \\
v\left(\delta k_{x}+i \delta k_{y}\right) & 0
\end{array}\right]\left[\begin{array}{c}
a_{k \sigma} \\
b_{k \sigma}
\end{array}\right],
$$


from which we arrive at the following effective Hamiltonian near the K (or K') point in the first quantization

$$
H_{0}=v \boldsymbol{\sigma} \cdot \boldsymbol{k}
$$

where $\boldsymbol{\sigma}=\left(\sigma_{x}, \sigma_{y}, \sigma_{z}\right)$ is the set of Pauli matrices describing the A and B sublattice (i.e., pseudospin) degrees of freedom, and we have changed the notation for the momentum from $\delta \boldsymbol{k}$ back to $\boldsymbol{k}$ understanding that $\boldsymbol{k}$ is now measured from the K (or $\left.\mathrm{K}^{\prime}\right)$ point. In real space, we substitute $\boldsymbol{k}=-i \nabla$. Diagonalization of this Hamiltonian in the momentum space leads to the two energy eigenvalues $\epsilon_{k \lambda}=\lambda v k ; \lambda= \pm 1$ is often referred to as the chirality in the graphene literature, with $\lambda=1$ describing the conduction band and $\lambda=-1$ the valence band. The two corresponding energy eigenstates are $|\boldsymbol{k} \lambda\rangle=\left[\begin{array}{ll}1 & \lambda e^{i \phi_{k}}\end{array}\right] / \sqrt{2}$, where $\phi_{\boldsymbol{k}}=\tan ^{-1}\left(k_{y} / k_{x}\right)$ is the azithmuthal angle of the momentm $\boldsymbol{k}$. We also note, for discussions in subsequent chapters, that the transformation which diagonalizes the Hamiltonian $H_{0}$ has the following form and is dependent on $\phi_{\boldsymbol{k}}$ :

$$
U_{\boldsymbol{k}}=\frac{1}{\sqrt{2}}\left[\begin{array}{cc}
1 & 1 \\
e^{i \phi_{\boldsymbol{k}}} & -e^{i \phi_{\boldsymbol{k}}}
\end{array}\right]
$$

Fig. 1.3 shows the full energy dispersion surface of graphene based on singleparticle band structure calculation, from which one can see that in the neighbourhood of the Brillouin zone corners $\mathrm{K}$ and $\mathrm{K}^{\prime}$ the energy dispersion indeed has a cone structure. The energy dispersion of graphene is thus linear with respect to momentum in the vicinity of these $\mathrm{K}$ and $\mathrm{K}^{\prime}$ points described by Eq. (1.10), with the upper portion of the cone behaving as the conduction band and the lower portion 
the valence band. In this vein, graphene can be regarded as a zero-gap semiconductor with a linear energy dispersion instead of the usual quadratic energy dispersion associated with regular semiconductors. Moreover, because the conduction and valence bands are connected at a single point, electrons in the valence band can also contribute to electronic properties through interband excitations to the conduction band. As in a semiconductor, graphene can be doped by chemical disposition of extrinsic atoms (such as potassium) or by electrical gating, with n-doping (addition of electrons) yielding a finite Fermi surface in the conduction band and p-doping (depletion of electrons) yielding a finite Fermi surface in the valence band. To carry the analogy with semiconductor even further, doped graphene (either by chemical doping or by electrical gating) will be called extrinsic graphene while undoped graphene called intrinsic graphene. Because of Eq. (1.11), the behavior of electron

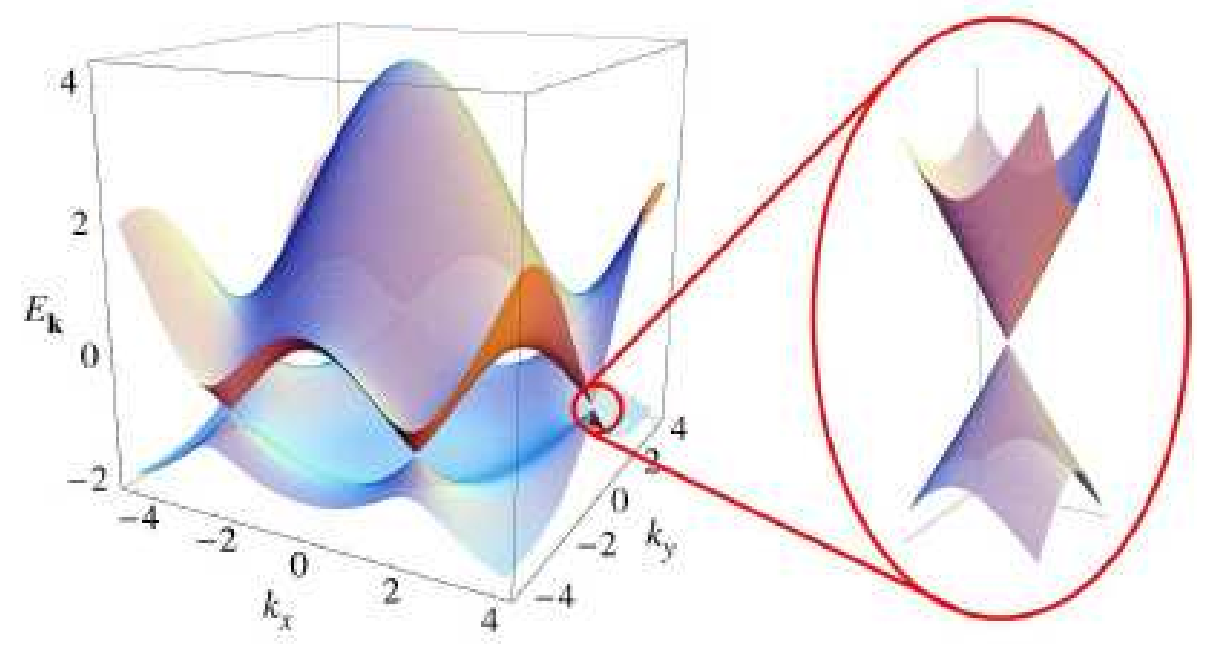

Figure 1.3: The energy-momentum dispersion surface of graphene (adapted from Fig. 3 of Ref. [1]). The zoom-in figure depicts the energy dispersion in the vicinity of the $\mathrm{K}$ point, which exhibits a cone-like structure.

in the vicinity of the $\mathrm{K}$ and $\mathrm{K}^{\prime}$ points shares the same physics with the behavior of 
the massless neutrino, which is governed by the massless Dirac equation. Electron in graphene therefore behaves similarly as massless neutrino, with its quantum mechanical motion governed by the Dirac equation instead of the Schrödinger equation. The electron pseudospin is analogous to the real spin in neutrinos pointing parallel or anti-parallel to the momentum direction $\boldsymbol{k}$. The Fermi velocity of graphene

electrons plays the role of the speed of light $c$, its value $v \approx 10^{6} \mathrm{~m}^{-1}$ being three hundred times smaller than $c$. Because of this remarkable property for the electrons near the $\mathrm{K}$ and $\mathrm{K}^{\prime}$ points, these points are also referred to as the Dirac points, and the electrons as Dirac fermions.

\subsubsection{Important graphene parameters}

In this section we introduce some important quantities that are relevant to our discussions later on in this dissertation. First, the electron energy dispersion in graphene is not all the way linear up from the Dirac point; at a momentum sufficiently far away from the Dirac point the energy dispersion becomes nonlinear as shown in Fig. 1.3. The energy at which this happens is given by the size of the Brillouin zone, and hence we define the cut-off momentum $k_{c}=\hbar / a$ (or equivalently, the cut-off energy $\Lambda_{c}=\hbar v / a$ where $a=1.42 \AA$ is the graphene lattice spacing) characterizing the region where the linear energy dispersion is valid. Second, from the graphene energy dispersion one can calculate the density of states as a function of energy as

$$
\nu(\varepsilon)=\frac{4 \varepsilon}{2 \pi \hbar^{2} v^{2}}
$$


where the factor of 4 counts the two spin and two valley degeneracies. We recall that the density of states of a regular two-dimensional electron gas (2DEG) with parabolic dispersion is a constant $\nu=m / \hbar^{2} \pi$; in contrast, the density of states of graphene varies linearly with energy and vanishes at the Dirac point $\varepsilon=0$. We note that the electron/hole density achievable by electrical back gating in experiment [18] is $n / p \sim 10^{14} \mathrm{~cm}^{-2}$.

In Chapter 2 and Chapter 3, we will consider Coulomb interaction in graphene. The relevant quantity indicating the strength of Coulomb interaction is given by the interaction coupling parameter $r_{s}$, which is defined as the ratio of the interparticle potential energy to the single-particle kinetic energy. We recall that for regular 2DEG $r_{s}=\left(e^{2} k_{F} / \epsilon_{m}\right) /\left(\hbar^{2} k_{F}^{2} / 2 m\right)=2 m e^{2} / \epsilon_{m} \hbar^{2} k_{F}$ is inversely proportional to the square root of density. For graphene,

$$
r_{s}=\frac{e^{2} k_{F} / \epsilon_{m}}{\hbar v k_{F}}=\frac{e^{2}}{\epsilon_{m} \hbar v}
$$

which is a constant independent of electron density, a result peculiar to the linear energy dispersion of graphene. Note that $r_{s}$ is analogous to one of Nature's fundamental constants - the fine structure constant $\alpha_{\mathrm{f}}=e^{2} / \hbar c$. For graphene on a $\mathrm{SiO}_{2}$ substrate, the background dielectric constant $\epsilon_{m}$ is obtained as the average of the vacuum and the substrate dielectric constants $\epsilon_{m}=(1+3.9) / 2=2.5$, and we have $r_{s} \simeq 0.87$

In Chapter 4 and Chapter 5, we will consider electron-phonon interaction in graphene. In particular, we are interested in the $\mathrm{G}$ band in-plane optical phonon which has an energy $\omega_{0}=200 \mathrm{meV}[13,5]$, and the electron-phonon interaction is 
characterized by the coupling constant [Eq. (4.4)]:

$$
g=-\left(\frac{\beta \hbar v}{a^{2}}\right) \sqrt{\frac{\hbar}{2 N M_{c} \omega_{0}}},
$$

with $N$ here the number of unit cells, $M_{c}=2.2 \times 10^{4} m_{e}$ the mass of a carbon atom ( $m_{e}$ is the electron mass), $a=1.42 \AA$ the equilibrium bond length between adjacent carbon atoms. The bond length oscillates around the equilibrium value $a$ due to lattice vibrations, and $\beta=\mathrm{d} \ln t / \mathrm{d} \ln l \sim 2$ is a dimensionless parameter describing the change of the nearest-neighbour hopping energy $t$ with respect to the bond length $l[19,20]$. In the presence of electron-phonon coupling, electrons can interact with one another through exchange of phonons, and this phonon-mediated electron-electron interaction is characterized by the dimensionless coupling constant [Eq. (4.22)]:

$$
\begin{aligned}
g_{\mathrm{ee}} & =\frac{g^{2} \mathcal{A}}{\hbar^{2} v^{2}}=\left(\frac{\beta}{a^{2}}\right)^{2} \frac{\hbar}{\rho \omega_{0}} \\
& =2 \times 10^{-2}
\end{aligned}
$$

where $\mathcal{A}$ is the sample area and $\rho$ is the mass density of graphene. The fact that both $r_{s}$ and $g_{\text {ee }}$ are smaller than unity shows that graphene is a weakly-interacting system, with the phonon-induced electron-electron coupling weaker than Coulomb coupling by an order of magnitude. 


\subsection{Angle-resolved photoemission spectroscopy}

\section{(ARPES)}

One important experimental technique to study the band structure of a material is angle-resolved photoemission spectroscopy (ARPES). The principle of ARPES is based on the photoelectric effect: electrons can be ejected from a material by absorbing photons of high enough energy. Upon absorbing a photon with an amount of energy $h \nu$ enough to overcome the binding forces in the solid, an electron in the bulk of the solid will have sufficient energy to escape from the surface of the material, with the amount of energy left going to the electron final-state kinetic energy $E_{\text {kin }}$ in the vacuum. The photon has to provide energy to do work on the electron to overcome the binding energy $E_{\mathrm{B}}$ of the electron in the solid and the surface potential barrier of energy $W$ (called the material work function), and therefore

$$
h \nu=\left|E_{\mathrm{B}}\right|+W+E_{\mathrm{kin}} .
$$

The goal is to determine the binding energy $E_{\mathrm{B}}$ of the electron inside the solid, and therefore by measuring the kinetic energy of the photo-emitted electron and the material work function $W$ one can obtain $E_{\mathrm{B}}$ from Eq. (1.18). Fig. 1.4 shows the geometry of the ARPES experiment. The magnitude of the momentum of the photo-emitted electron is given by its kinetic energy as $p=\sqrt{2 m E_{\text {kin }}}$. In the case of a two-dimensional system (for which graphene is a prominent example), one needs to concern only the in-plane component of the electron momentum inside the material, which can be deduced from conservation of momentum as $p_{/ /}=\sqrt{2 m E_{\mathrm{kin}}} \sin \theta$, 
where $\theta$ is the polar angle at which the photo-emitted electron is detected in the vacuum. The energy-momentum relationship can, therefore, be mapped in this way for different directions. On the other hand, the energy dispersion can also be more simply mapped out by tracking the energy position of the peaks detected in the ARPES spectra as a function of $p_{/ /}$for different directions. Besides the energy

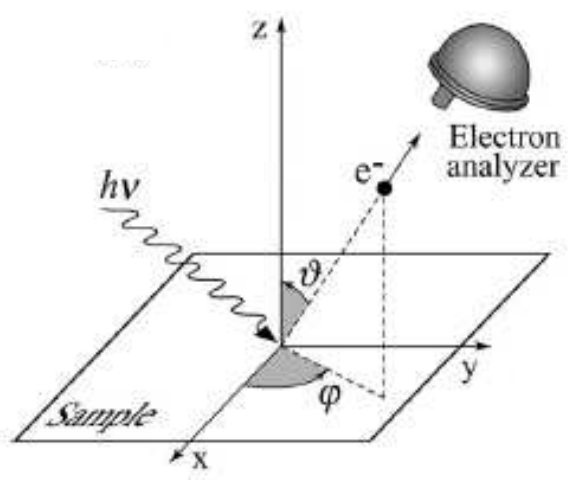

Figure 1.4: Geometry of an ARPES experiment. (adapted from Fig. 6(a) of Ref. [2]) The electron analyzer registers the photoemission intensity at a polar angle $\theta$ and azithmuthal angle $\phi$.

dispersion of a material, another important quantity which can be extracted from the ARPES data is the quasiparticle spectral function $A_{\boldsymbol{k}}(\omega)$. The spectral function is an important quantity because it provides a measure of how well the elementary excitations in a material can be described by quasiparticles within the framework of Fermi liquid theory. For non-interacting particles, the spectral function is given by a delta function at the particle energy, whereas for interacting particles the spectral function broadens into a Lorentian with the center position and the line-width giving information of the interparticle interaction. In the language of many-body theory, the spectral function of a quasiparticle having energy $\xi_{k}=\epsilon_{k}-\varepsilon_{F}$ is defined as $A_{\boldsymbol{k}}(\omega) \equiv-2 \operatorname{Im} G_{k}(\omega)$, where $G_{k}(\omega)$ is the retarded interacting quasiparticle Green 
function given by $G_{k}(\omega)=1 /\left[\omega-\xi_{k}-\Sigma_{k}(\omega)\right]$. The quantity $\Sigma_{k}(\omega)$ is the retarded self-energy, which we will consider in detail in the following chapters. The measured photoemission intensity $I(\boldsymbol{k}, \omega)$ is directly proportional to the spectral function:

$$
\begin{aligned}
I(\boldsymbol{k}, \omega) & \propto n_{F}(\omega) A_{\boldsymbol{k}}(\omega) \\
& =n_{F}(\omega) \frac{\operatorname{Im} \Sigma_{k}(\omega)}{\left[\operatorname{Im} \Sigma_{k}(\omega)\right]^{2}+\left[\omega-\operatorname{Re} \Sigma_{k}(\omega)\right]^{2}},
\end{aligned}
$$

where $n_{F}(\omega)=1 /\left[\exp \left(\omega / k_{\mathrm{B}} T\right)+1\right]$ is the Fermi distribution function.

ARPES studies of the spectral function have been carried out previously in metals and cuprate materials (see, for example, Refs. [21] and [22]). Information on the spectral function can be directly extracted from scanning the measured ARPES intensity as a function of energy at constant values of momentum, resulting in socalled energy distribution curves (EDCs), and as a function of momentum at constant values of energy, resulting in so-called momentum distribution curves (MDCs). The EDCs is usually not as useful as the MDCs, because the self-energy generally has a complicated dependence on energy $\omega$ resulting a complex line-shape of the EDCs. On the other hand, at constant values of energy MDCs are very well approximated by Lorentzians assuming a momentum-independent self-energy, making them very useful for extracting the imaginary part of the self-energy by measuring the line-width of the Lorentzian. Fig. 1.5 shows typical EDCs and MDCs obtained in ARPES experiments. 

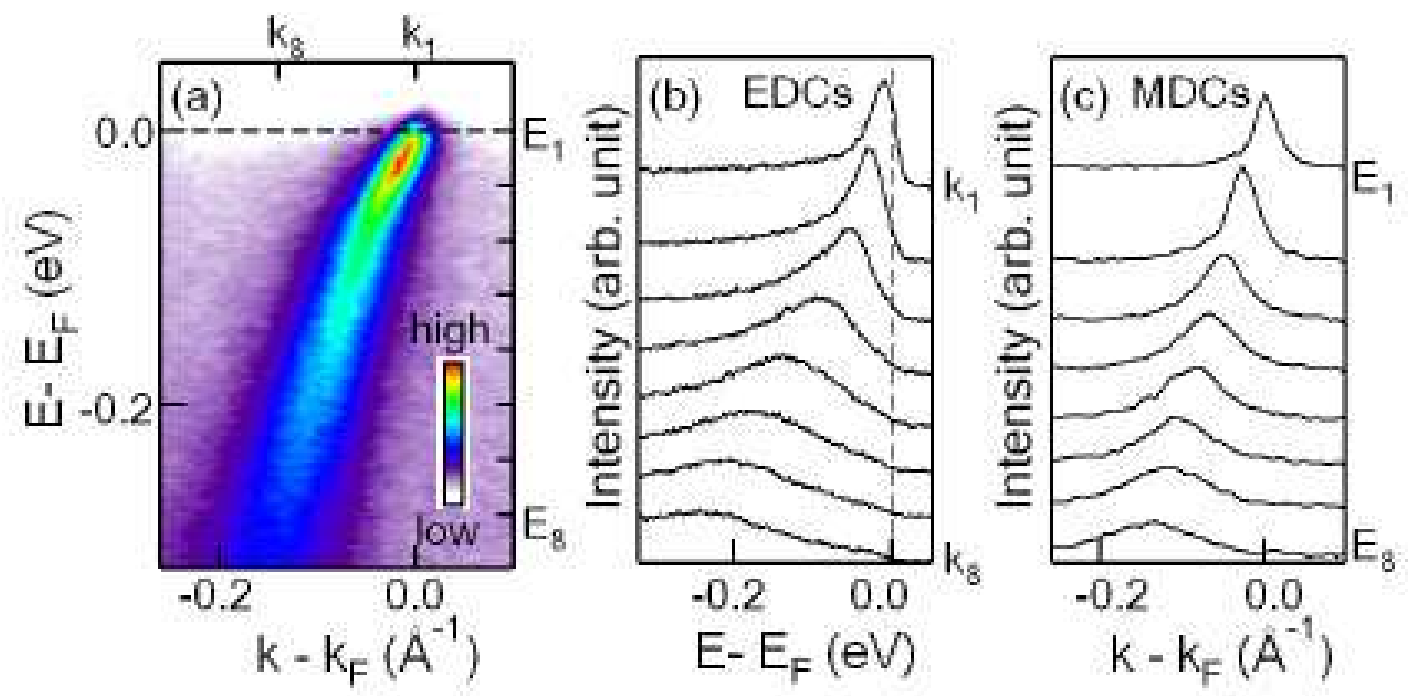

Figure 1.5: ARPES intensity (a) EDCs, (b) MDCs and (c) measured on the material Bi2212 (adapted from Ref. [3]). The EDCs are shown vertically displaced for different values of momentum and the MDCs for different values of energy.

\subsubsection{Graphene ARPES experiment}

Using APRES, the linear energy spectrum of Dirac fermions was first directly observed by the group of A. Lanzara of Berkeley with a graphite sample [23] and by the group of E. Rotenberg of the Lawrence Berkeley National Laboratory with a graphene sample [4]. Since then, more ARPES experiments on graphene have emerged $[24,25,26,27]$. In this section we provide a review on the first ARPES experiment on graphene performed by the group of E. Rotenberg [4]. The experiment was performed on a single layer of graphene grown on a $\mathrm{SiC}$ substrate, and the graphene Fermi level is changed by chemical doping with potassium atoms. Fig. 1.6 shows the constant energy surfaces extracted from the ARPES intensity, from which the Fermi level around the six Brillouin zone corners (i.e., the $\mathrm{K}$ and K' points) can be clearly distinguished. The dispersion relation obtained from the 


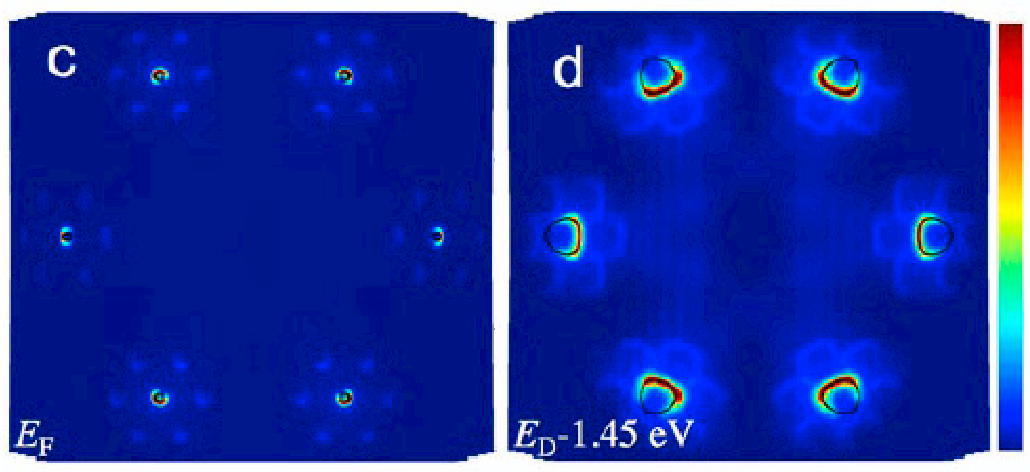

Figure 1.6: Constant energy maps adapted from Fig. 1c-d of Ref. [4], in which the Fermi level around the six $\mathrm{K}$ and $\mathrm{K}$ ' points are clearly discernible. Fig. 1.6 c corresponds to an electron doping with a Fermi level of $0.45 \mathrm{eV}$ above the Dirac point and Fig. 1d to a hole doping with a Fermi level of $1.5 \mathrm{eV}$ below the Dirac point. It can be seen that from Fig. 1.6 d that at such a large energy away from the Dirac point the energy dispersion is no longer linear.

experiment is shown in Fig. 1.7 with different levels of doping, from which a welldefined linear energy-momentum relationship is observed, experimentally verifying the theoretical prediction that electrons in graphene in the vicinity of the Brillouin zone corners are indeed Dirac-like. There are, however, subtle features observed in the band structure: 1. There are line-shape distortions from the linear dispersion; in particular, there are two kinks occurring at the Dirac point (indicated by the first arrow $E_{\mathrm{D}}$ in Fig. $1.7 \mathrm{e}-\mathrm{h}$ ) and at about $200 \mathrm{meV}$ (indicated by the second arrow $\omega_{\text {ph }}$ in Fig. 1.7 e-h) away from the Fermi level; 2. there are line-width variations in the measured photoemission intensity. To explain these features, one must go beyond the single-particle tight-binding description of graphene and take into account the many-body interactions of the electrons. The line-width distortion of the band structure is then accounted for by the renormalization of the single-particle energy by the real part of the self-energy, whereas the line-width is due to the finite lifetime of the quasiparticle given by the imaginary part of the self-energy. 

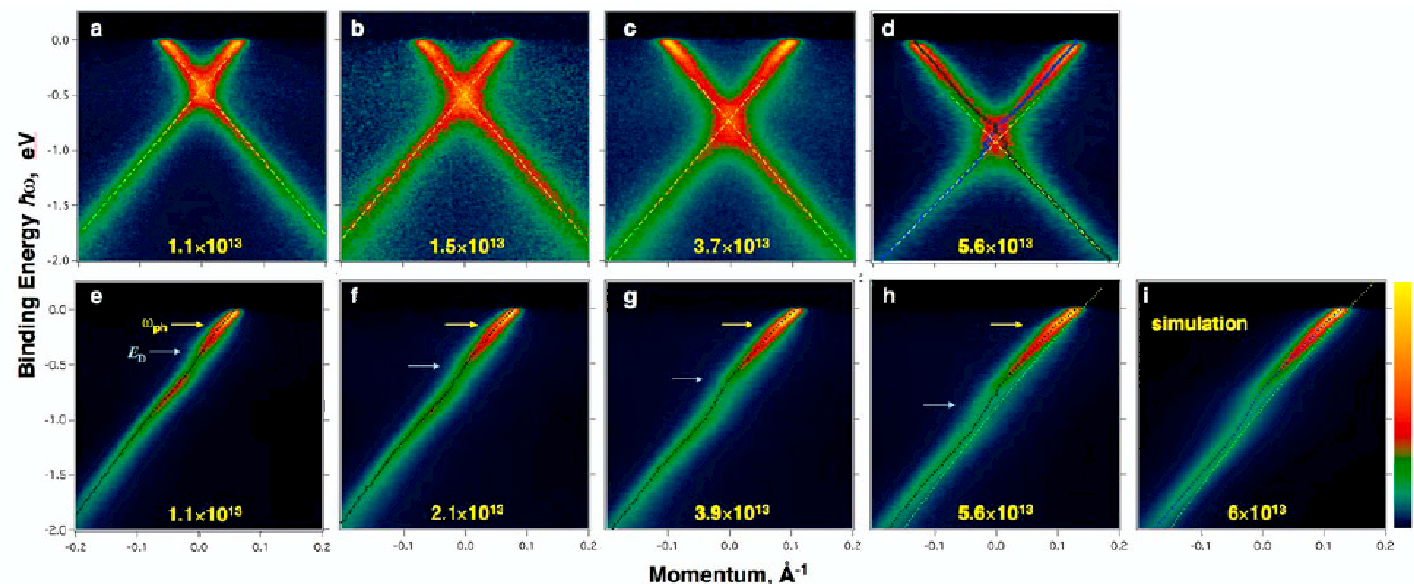

Figure 1.7: Band structure of graphene at the Brillouin zone corner K adapted from Fig. 2 of Ref. [4]. The colored plots show the measured intensity, and the dashed lines indicate an extrapolation of the lower band. The number in each subfigure shows the electron density in $\mathrm{cm}^{-2}$. Fig. $1.7 \mathrm{i}$ shows the reconstructed spectral function using the extracted imaginary part of the self-energy from the line-width of MDCs.

Approximating the self-energy as momentum-independent, Ref. [4] extracted the imaginary part of the self-energy by measuring the line-width of the MDCs, which are shown in Fig. 1.8. The real part of the self-energy was then determined by a Kramers-Kronig analysis, and the reconstructed real and imaginary parts of the self-energy were used to calculate the spectral function, which shows a good agreement with the measured ARPES intensity (Fig. 1.7i).

\subsection{Outline of the thesis}

In this dissertation, we present a systematic study of the many-body effects on the electronic and transport properties of graphene. In Chapter 2, we consider the effect of Coulomb interaction on the renormalization of the electron properties in graphene. We take into account screening effects using the Random-Phase 


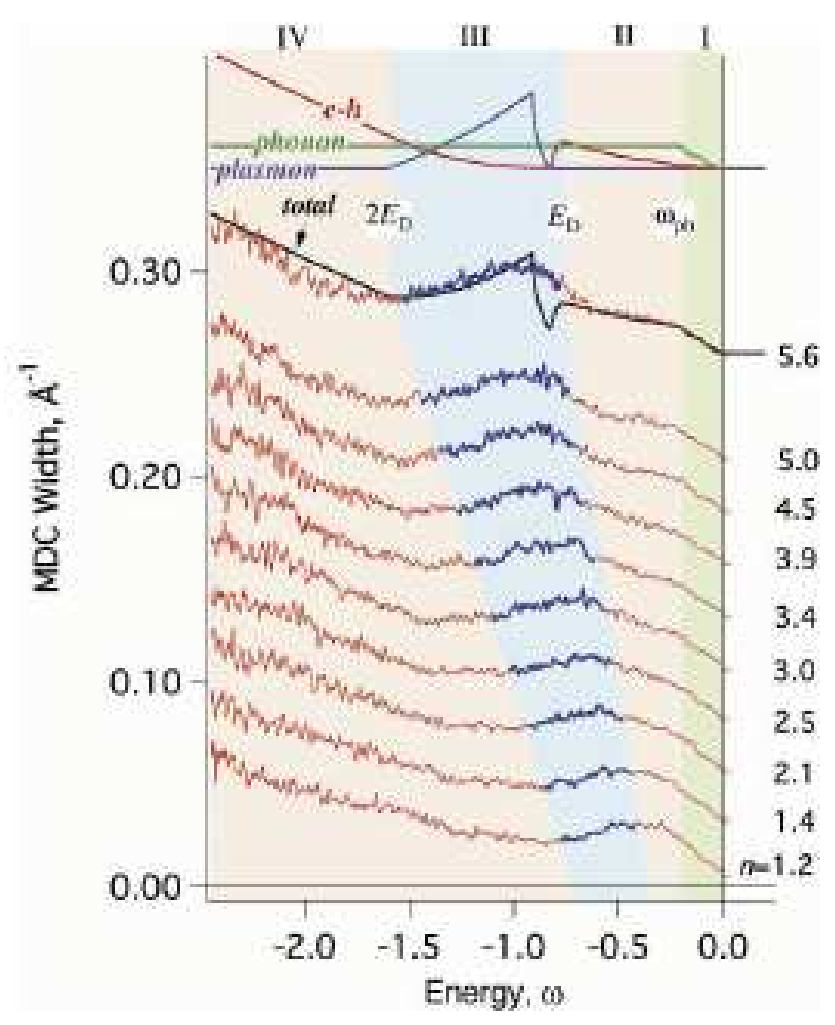

Figure 1.8: Measured line-widths of the MDCs at different values of electron density $n$ (in units of $10^{13} \mathrm{~cm}^{-2}$ ) from Ref. [4].

Approximation, formulate the electron self-energy problem and then calculate analytically the renormalized quaisparticle properties, namely quasiparticle decay rate, renormalization factor, and renormalized velocity for both extrinsic and intrinsic graphene. The work in this chapter has led to the publication of Ref. [28].

In Chapter 3, we consider the effect of many-body Coulomb interaction on the transport properties of a graphene double-layer system. While most transport properties are manifestations of single electron properties, Coulomb drag is, by contrast, the only transport phenomenon that is directly induced by electron-electron interaction whose effect can be measured directly in experiment. We formulate the problem for the drag resistivity in graphene within the linear response diagrammatic 
approach, and take into account screening of the interlayer Coulomb interaction within the random-phase approximation. We then calculate the drag resistivity as a function of temperature, electron densities and interlayer distance. The work in this chapter has led to the publication of Ref. [29].

We have discussed the effect of Coulomb interaction on graphene electronic properties in Chapter 2. In Chapter 4, we consider the effect of electron-phonon interaction on graphene electronic properties. In carbon nanotubes, electron-phonon interaction is a well-studied subject, The motivation of this study came from the ARPES band structure measurement where a kink whose origin was speculated to be electron-phonon interaction was observed. Starting from the graphene electronphonon interaction, we derive the expression for the effective phonon-mediated electron-electron interaction and formulate the electron self-energy problem. We then calculate exactly the imaginary and real parts of the self-energy, and obtain the quasiparticle spectral function and renormalized velocity. The work in this chapter has led to the publication of Ref. [30].

In Chapter 2 and 4, we have considered the renormalization of the electron properties due to Coulomb and electron-phonon interactions; in Chapter 5, we ask the question how interaction effects will renormalize the phonon properties. We formulate the phonon self-energy problem, and calculate the real part of the phonon self-energy. We then obtain the renormalized phonon energy dispersion, finding novel Kohn anomalies peculiar to optical phonons in graphene which are completely different from $q=2 k_{F}$ in regular metals. The work in this chapter has led to the publication of Ref. [31]. 
In Chapter 6, we present our conclusion and finally discuss some open questions for future exploration extending the research in this dissertation. 


\section{Chapter 2}

\section{Quasiparticle renormalization due}

\section{to Coulomb interaction}

One of the most important concepts in condensed matter physics is Fermi liquid theory developed by Landau, which establishes a one-to-one correspondence between the properties of an interacting electron liquid and a non-interacting electron gas. For interactions that are weak enough, interacting electrons behave as non-interacting dressed particles - called quasiparticles - that are elementary excitations from the ground state (which is the Fermi sea in a solid). These quasiparticles behave similarly to non-interacting electrons, except that their properties are renormalized compared with the non-interacting electron properties. One of the main goals of many-body theory has been to calculate these renormalized quasiparticle parameters, namely the quasiparticle lifetime, renormalization factor and renormalized mass/velocity; and it is our goal in this chapter to develop a self-energy 
formulation to calculate these quantities. The Hamiltonian of our system is

$$
H=\sum_{\boldsymbol{k}} \boldsymbol{c}_{\boldsymbol{k}}^{\dagger} H_{0} \boldsymbol{c}_{\boldsymbol{k}}+\frac{1}{2} \sum_{\boldsymbol{k}_{1}, \boldsymbol{k}_{2}, \boldsymbol{q}} V(q) \boldsymbol{c}_{\boldsymbol{k}_{1}+\boldsymbol{q}}^{\dagger} \boldsymbol{c}_{\boldsymbol{k}_{1}} \boldsymbol{c}_{\boldsymbol{k}_{2}-\boldsymbol{q}}^{\dagger} \boldsymbol{c}_{\boldsymbol{k}_{2}}
$$

where $\boldsymbol{c}_{\boldsymbol{k}}=\left[\begin{array}{ll}a_{\boldsymbol{k}} & b_{\boldsymbol{k}}\end{array}\right]^{\mathrm{T}}$ is the two-component electron annihilation operator in the

momentum space for the $\mathrm{A}$ and $\mathrm{B}$ sublattices, and $V(q)=2 \pi e^{2} / q$ is the bare Coulomb interaction. We have suppressed the spin labels in Eq. (2.1) and will continue to do so in the rest of this dissertation since the spin degrees of freedom are understood to be degenerate and do not play a role in the physics we consider. However, we shall keep in mind to restore the factor of two for spin degeneracy as well as the factor of two for valley degeneracy in the density of states and all closed-loop diagrams.

\subsection{Random-phase approximation}

In a many-electron system, electron-electron Coulomb interaction is screened by the surrounding electrons, with the consequence that both the strength and the range of the electron-electron interaction are reduced. Within the diagrammatic perturbation theory, a standard approximation to take account of the effect of screening is the Random-phase approximation (RPA) $[32,33,34]$, which retains only the most divergent (and hence having the largest contribution) bubble diagrams in the diagrammatic expansion. The resulting screened Coulomb interaction is thus given by the bare Coulomb interaction dressed by a series of bubble diagrams, as shown in Fig. 2.1. Summing the diagrammatic series yields the dynamically screened Coulomb 


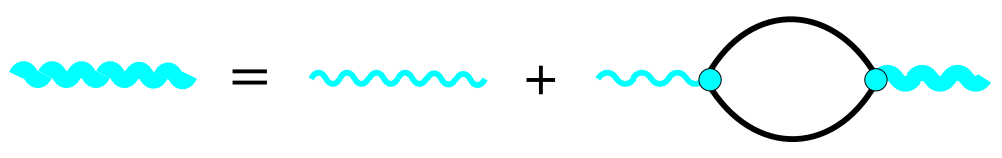

Figure 2.1: The Feynman diagram for the screened Coulomb interaction within the RPA. The thin and thick wiggly lines denote respectively the bare and the RPAscreened interaction, and the thin straight line stands for the bare Green function.

interaction

$$
V_{\mathrm{sc}}(q, \omega)=\frac{V(q)}{1-V(q) \prod_{0}^{\mathrm{cc}}(q, \omega)}
$$

where $\Pi_{0}^{\mathrm{cc}}$ is the irreducible electronic polarizability represented by the bubble diagram with two Coulomb interaction vertices (the superscript 'c' denotes a Coulomb interaction vertex, and the subscript '0' denotes irreducibility). We shall drop the subscript ' 0 ' in $\Pi_{0}^{c c}$, understanding that we are referring to the irreducible polarizability (when we come to Chapter 5, we will restore the subscript '0' again to emphasize it is a "bare" or unrenormalized bubble). $\Pi^{\text {cc }}$ is given from the bubble diagram (Fig. 2.1) by the following expression

$$
\begin{aligned}
\Pi^{\mathrm{cc}}\left(q, i q_{n}\right) & =4 k_{\mathrm{B}} T \sum_{\lambda \lambda^{\prime}} \sum_{k, i k_{n}} G_{k+q \lambda^{\prime}}\left(i k_{n}+i q_{n}\right) G_{k \lambda}\left(i k_{n}\right)\left\langle\boldsymbol{k}+\boldsymbol{q} \lambda^{\prime} \mid \boldsymbol{k} \lambda\right\rangle\left\langle\boldsymbol{k} \lambda \mid \boldsymbol{k}+\boldsymbol{q} \lambda^{\prime}\right\rangle \\
& =4 k_{\mathrm{B}} T \sum_{\lambda \lambda^{\prime}} \sum_{k, i k_{n}} G_{k+q \lambda^{\prime}}\left(i k_{n}+i q_{n}\right) G_{k \lambda}\left(i k_{n}\right) \frac{1+\lambda \lambda^{\prime} \cos \left(\phi_{k+q}-\phi_{k}\right)}{2}(2.3)
\end{aligned}
$$

Carrying out the Matsubara sum over $i k_{n}$, we obtain

$$
\Pi^{\mathrm{cc}}\left(q, i q_{n}\right)=4 \sum_{\lambda \lambda^{\prime}} \sum_{k} \frac{n_{F}\left(\xi_{k \lambda}\right)-n_{F}\left(\xi_{k+q \lambda^{\prime}}\right)}{i q_{n}+\xi_{k \lambda}-\xi_{k+q \lambda^{\prime}}} \frac{1+\lambda \lambda^{\prime} \cos \left(\phi_{k+q}-\phi_{k}\right)}{2} .
$$


Eq. (2.2) defines the dielectric function, given by $\epsilon\left(q, i q_{n}\right)=1-V(q) \Pi^{\mathrm{cc}}\left(q, i q_{n}\right)$ within the RPA. The analytic expression of $\Pi^{c c}$ has been obtained in a number of papers $[6,35,36,37]$, which is reproduced here for expedient reference [6]. The polarizability consists of two contributions $\Pi^{\mathrm{cc}}=\Pi_{+}^{\mathrm{cc}}+\Pi_{-}^{\mathrm{cc}}$, where $\Pi_{-}^{\mathrm{cc}}$ is due to the intrinsic electrons filled up to the Dirac point in the valence band and $\Pi_{+}^{\mathrm{cc}}$ is due to the extrinsic electrons in the conduction band from n-doping or holes in the valence band from p-doping. In the following, we give the results for the polarizability separately for the real part and the imaginary part, understanding that the analytic continuation of the Matsubara frequency $i q_{n} \rightarrow \omega+i 0^{+}$has been carried out [6]. We also define, for notational simplicity, the dimensionless momentum $x=q / k_{F}$, energy $u=\omega / \varepsilon_{F}$, and polarizability $\tilde{\Pi}^{\mathrm{cc}}=\Pi^{\mathrm{cc}} / \nu\left(\varepsilon_{F}\right)$, where $\nu\left(\varepsilon_{F}\right)=2 k_{F} / \pi v$ is the graphene density of states at the Fermi level.The extrinsic contribution to the polarizability $\tilde{\Pi}_{+}^{c c}$ is given by the sum of two contributions originating from intraband and interband transitions:

$$
\tilde{\Pi}_{+}^{\mathrm{cc}}(x, u)=\tilde{\Pi}_{+}^{\mathrm{inter}}(x, u) \theta(u-x)+\tilde{\Pi}_{+}^{\mathrm{intra}}(x, u) \theta(x-u),
$$

where the real and imaginary parts are given by,

$$
\begin{gathered}
\operatorname{Re} \tilde{\Pi}_{+}^{\text {inter }}(x, u)= \\
+1+\frac{1}{8 \sqrt{u^{2}-x^{2}}}\left\{f_{1}(x, u) \theta(|2+u|-x)\right. \\
+\operatorname{sgn}(u-2+x) f_{1}(x,-u) \theta(|2-u|-x) \\
\left.+\quad f_{2}(x, u)[\theta(x+2-u)+\theta(2-x-u)]\right\} \\
\operatorname{Re} \tilde{\Pi}_{+}^{\text {intra }}(x, u)=-1+\frac{1}{8 \sqrt{x^{2}-u^{2}}}\left\{f_{3}(x, u) \theta(x-|u+2|)+f_{3}(x,-u) \theta(x-|u-2|)\right. \\
\left.+\frac{\pi x^{2}}{2}[\theta(|u+2|-x)+\theta(|u-2|-x)]\right\}
\end{gathered}
$$




$$
\begin{gathered}
\operatorname{Im} \tilde{\Pi}_{+}^{\text {inter }}(x, u)= \\
+\frac{1}{8 \sqrt{u^{2}-x^{2}}}\left\{f_{3}(x,-u) \theta(x-|u-2|)\right. \\
\left.+\frac{\pi x^{2}}{2}[\theta(x+2-u)+\theta(2-x-u)]\right\}, \\
\operatorname{Im} \tilde{\Pi}_{+}^{\text {intra }}(x, u)=-\frac{\theta(u-x+2)}{8 \sqrt{x^{2}-u^{2}}}\left[f_{4}(x, u)-f_{4}(x,-u) \theta(2-x-u)\right] .
\end{gathered}
$$

Here the functions $f_{1,2,3,4}$ are defined as

$$
\begin{gathered}
f_{1}(x, u)=(2+u) \sqrt{(2+u)^{2}-x^{2}}-x^{2} \ln \frac{\sqrt{(2+u)^{2}-x^{2}}+(2+u)}{\left|\sqrt{u^{2}-x^{2}}+u\right|} \\
f_{2}(x, u)=x^{2} \ln \frac{u-\sqrt{u^{2}-x^{2}}}{x} \\
f_{3}(x, u)=(2+u) \sqrt{x^{2}-(2+u)^{2}}+x^{2} \sin ^{-1} \frac{2+u}{x} \\
f_{4}(x, u)=(2+u) \sqrt{(2+u)^{2}-x^{2}}-x^{2} \ln \frac{\sqrt{(2+u)^{2}-x^{2}}+(2+u)}{x} .
\end{gathered}
$$

The intrinsic contribution to the polarizability $\tilde{\Pi}_{-}^{\mathrm{cc}}(x, u)$ is given simply by,

$$
\tilde{\Pi}_{-}^{\mathrm{cc}}(x, u)=-\frac{\pi x^{2} \theta(x-u)}{8 \sqrt{x^{2}-u^{2}}}-i \frac{\pi x^{2} \theta(u-x)}{8 \sqrt{u^{2}-x^{2}}} .
$$

\section{$2.2 \quad$ Self-energy}

We now calculate the quasiparticle self-energy in the leading order of the dynamically screened Coulomb interaction (also called the $\mathrm{G}_{0} \mathrm{~W}$ approximation) [38], represented diagrammatically in Fig. 2.2. Since we are interested in the self-energy for electrons in the conduction band or holes in the valence band, we evaluate the self-energy in the chiral basis (i.e., the basis where $H_{0}$ is diagonal) $\Sigma_{ \pm}$, where the subscript \pm denotes the chirality and refers to electrons in the conduction band $(+)$ 


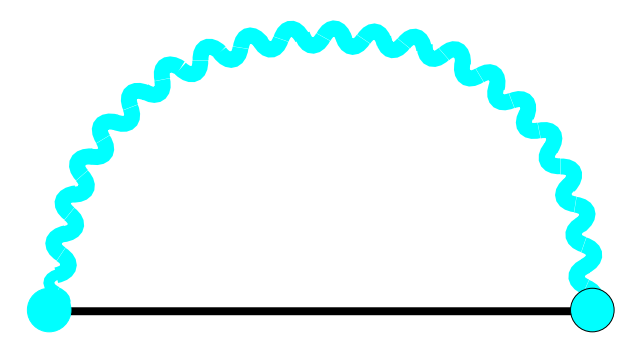

Figure 2.2: The electron self-energy $\Sigma$ Eq. (2.15), the thick wiggly line denotes the screened Coulomb interaction within the RPA in Fig. 2.1. This is the standard ring diagram approximation for self-energy exact in the $r_{s} \ll 1$ limit.

or holes in the valence band (-). The quasiparticle self-energy in the chiral basis can be written down from Fig. 2.2 as

$$
\left[\Sigma_{\boldsymbol{k}}\left(i k_{n}\right)\right]_{\mu^{\prime} \lambda}=-k_{\mathrm{B}} T \sum_{\boldsymbol{q}, i q_{n}} G_{\boldsymbol{k}+\boldsymbol{q} \mu}^{0}\left(i k_{n}+i q_{n}\right) \frac{V_{q}}{\epsilon\left(q, i q_{n}\right)}\left\langle\boldsymbol{k}+\boldsymbol{q} \mu \mid \boldsymbol{k} \mu^{\prime}\right\rangle\left\langle\boldsymbol{k} \lambda \mid \boldsymbol{k}+\boldsymbol{q} \lambda^{\prime}\right\rangle \delta_{\mu \lambda^{\prime}}
$$

where $G_{k \lambda}^{0}\left(i k_{n}\right)=1 /\left(i k_{n}-\xi_{k \lambda}\right)$ is the bare quasiparticle Green function, $\xi_{k \lambda}=$ $\lambda \varepsilon_{k}-\mu=\lambda v k-\mu$ is the single-particle energy ( $\mu$ here is the chemical potential), $V_{q}=2 \pi e^{2} / q$ is the bare Coulomb interaction, and $\epsilon\left(q, i q_{n}\right)$ is the dielectric function. Summation over repeated indices is understood. The matrix elements in Eq. (2.15) (i.e., the vertices in the self-energy diagram) follow straightforwardly from the eigenstates of $H_{0}$ as

$$
\begin{aligned}
\left\langle\boldsymbol{k} \lambda \mid \boldsymbol{k}+\boldsymbol{q} \lambda^{\prime}\right\rangle & =\frac{1}{2}\left[1+\lambda \lambda^{\prime} e^{i\left(\phi_{\boldsymbol{k}+\boldsymbol{q}}-\phi_{\boldsymbol{k}}\right)}\right] \\
\left\langle\boldsymbol{k}+\boldsymbol{q} \mu \mid \boldsymbol{k} \mu^{\prime}\right\rangle & =\frac{1}{2}\left[1+\mu \mu^{\prime} e^{-i\left(\phi_{\boldsymbol{k}+\boldsymbol{q}}-\phi_{\boldsymbol{k}}\right)}\right]
\end{aligned}
$$

where $\phi_{\boldsymbol{k}}$ is the azithmuthal angle of the momentum $\boldsymbol{k}$. Eqs. (2.15)-(2.16) then give the diagonal elements of the self-energy matrix $\Sigma_{\boldsymbol{k} \pm}\left(i k_{n}\right) \equiv \Sigma_{\boldsymbol{k} \pm \pm}\left(i k_{n}\right)$

$$
\Sigma_{\boldsymbol{k} \pm}\left(i k_{n}\right)=-k_{\mathrm{B}} T \sum_{\lambda= \pm \boldsymbol{q}, i q_{n}} G_{\boldsymbol{k}+\boldsymbol{q} \lambda}^{0}\left(i k_{n}+i q_{n}\right) \frac{V_{q}}{\epsilon\left(q, i q_{n}\right)} \frac{1 \pm \lambda \cos \left(\phi_{\boldsymbol{k}+\boldsymbol{q}}-\phi_{\boldsymbol{k}}\right)}{2}
$$


and the off-diagonal elements of the self-energy matrix

$$
\Sigma_{\boldsymbol{k} \pm \mp}\left(i k_{n}\right)= \pm i k_{\mathrm{B}} T \sum_{\lambda= \pm} \sum_{\boldsymbol{q}, i q_{n}} G_{\boldsymbol{k}+\boldsymbol{q} \lambda}^{0}\left(i k_{n}+i q_{n}\right) \frac{V_{q}}{\epsilon\left(q, i q_{n}\right)} \frac{\lambda \sin \left(\phi_{\boldsymbol{k}+\boldsymbol{q}}-\phi_{\boldsymbol{k}}\right)}{2}=0
$$

vanish identically after performing the angular integration. The self-energy correction therefore does not "mix" the electrons in the conduction band and the holes in the valence band. In Eq. (2.17), the factor $\left[1 \pm \lambda \cos \left(\phi_{\boldsymbol{k}+\boldsymbol{q}}-\phi_{\boldsymbol{k}}\right)\right] / 2$ originates from the chirality of the graphene band dispersion, with $\lambda=1$ corresponding to intraband transitions and $\lambda=-1$ to interband transitions. Decomposing Eq. (2.17) into line and pole contributions, we get

$$
\begin{aligned}
\Sigma_{\boldsymbol{k} \pm}\left(i k_{n}\right)= & -\frac{1}{2} \sum_{\lambda= \pm} \sum_{\boldsymbol{q}}\left[1 \pm \lambda \cos \left(\phi_{\boldsymbol{k}+\boldsymbol{q}}-\phi_{\boldsymbol{k}}\right)\right] V_{q}\left\{\mathrm{P} \int_{-\infty}^{\infty} \frac{\mathrm{d} \omega}{\pi} n_{B}(\omega) \operatorname{Im}\left[\frac{1}{\epsilon\left(q, \omega+i 0^{+}\right)}\right]\right. \\
& \left.\frac{1}{\omega+i k_{n}-\xi_{\boldsymbol{k}+\boldsymbol{q} \lambda}}+\frac{n_{F}\left(\xi_{\boldsymbol{k}+\boldsymbol{q} \lambda}\right)}{\epsilon\left(q, \xi_{\boldsymbol{k}+\boldsymbol{q} \lambda}-i k_{n}\right)}\right\}
\end{aligned}
$$

where $\mathrm{P}$ denotes the principal value of the integral, $n_{F}(\varepsilon)=1 /\left[\exp \left(\varepsilon / k_{B} T\right)+1\right]$ and $n_{B}(\varepsilon)=1 /\left[\exp \left(\varepsilon / k_{B} T\right)-1\right]$ are, respectively, the Fermi and Bose distribution functions. Performing the analytic continuation $i k_{n} \rightarrow \varepsilon+i 0^{+}$, we obtain the retarded self-energy

$$
\begin{aligned}
\Sigma_{\boldsymbol{k} \pm}^{\mathrm{R}}(\varepsilon)= & -\frac{1}{2} \sum_{\lambda= \pm} \sum_{\boldsymbol{q}}\left[1 \pm \lambda \cos \left(\phi_{\boldsymbol{k}+\boldsymbol{q}}-\phi_{\boldsymbol{k}}\right)\right] V_{q}\left\{\mathrm{P} \int_{-\infty}^{\infty} \frac{\mathrm{d} \omega}{\pi} n_{B}(\omega) \operatorname{Im}\left[\frac{1}{\epsilon\left(q, \omega+i 0^{+}\right)}\right]\right. \\
& \left.\frac{1}{\omega+\varepsilon-\xi_{\boldsymbol{k}+\boldsymbol{q} \lambda}+i 0^{+}}+\frac{n_{F}\left(\xi_{\boldsymbol{k}+\boldsymbol{q} \lambda}\right)}{\epsilon\left(q, \xi_{\boldsymbol{k}+\boldsymbol{q} \lambda}-\varepsilon-i 0^{+}\right)}\right\} .
\end{aligned}
$$

By virtue of Fermi liquid theory, in the presence of weak electron-electron interactions, the properties of the quasiparticles are renormalized with a finite quasiparticle lifetime $\tau$, a renormalization factor $Z<1$, and a renormalized mass $m^{*}$ or velocity $v^{*}$. In the following, we proceed to calculate the quasiparticle lifetime, 
renormalization factor and effective velocity at zero temperature for graphene in two distinct cases: extrinsic graphene with n-doping (Fermi energy $\varepsilon_{F}>0$ ) and intrinsic graphene $\left(\varepsilon_{F}=0\right)$. We recall the Coulomb interaction coupling parameter $r_{s}$ from Chapter 1 is defined by the ratio of the interparticle potential energy to the single-particle kinetic energy. It is worth noting that whereas for regular 2DEG, $r_{s}=2 m e^{2} / k_{F} \epsilon_{m}$ is inversely proportional to the square root of the $2 \mathrm{D}$ density, for graphene $r_{s}=\left(e^{2} k_{F} / \epsilon_{m}\right) / v k_{F}=e^{2} / v \epsilon_{m}$ is simply a constant $\sim 0.87$ (with $v \simeq 10^{6} \mathrm{~ms}^{-1}$ and $\epsilon_{m} \simeq 2.5$ ), indicating extrinsic graphene is essentially a weakly interacting $\left(r_{s}<1\right)$ system. In this context, we also note that ordinary metals have $r_{s} \simeq 3-5>1$, and the usual semiconductor-based 2DEG may have $r_{s} \sim 5-20 \gg 1$ ! Since $r_{s}<1$ in graphene, the self-energy (Fig. 2.2) calculation in the leading-order infinite ring-diagram single-loop expansion approximation is essentially exact. In fact, the RPA self-energy calculation (Fig. 2.2) is a much better quantitative approximation to graphene than the GW approximation in regular metals and semiconductors, which are never in the high-density regime.

\subsection{Extrinsic graphene}

For extrinsic graphene, the quasiparticle is located in the vicinity of the Fermi level, and we only need to consider the renormalized Fermi liquid parameters in the conduction band (or the valence band, depending on whether electrons or holes are the carriers as determined by doping). In the presence of interactions, the Fermi energy will be renormalized by the real part of the self-energy. We adopt 
the on-shell approximation, which implies that the Fermi energy is taken to be the unrenormalized Fermi energy $\varepsilon_{F}$ without any correction coming from the real part of the self-energy. This guarantees that our self-energy calculation within the $\mathrm{G}_{0} \mathrm{~W}$ approximation is internally consistent and different orders of the self-energy correction do not mix [39].

\subsubsection{Quasiparticle decay rate}

The lifetime of the quasiparticle $\tau_{+}=-1 / 2 \operatorname{Im} \Sigma_{\boldsymbol{k}+}^{\mathrm{R}}\left(\xi_{k}\right)$ is obtained from the imaginary part of the self-energy Eq. (2.17) within the on-shell approximation $\varepsilon=$ $\xi_{k}=v k-\varepsilon_{F}:$

$$
\begin{aligned}
\operatorname{Im} \Sigma_{\boldsymbol{k}+}^{\mathrm{R}}\left(\xi_{k}\right)= & \frac{1}{2} \sum_{\lambda= \pm} \sum_{\boldsymbol{q}}\left[1+\lambda \cos \left(\phi_{\boldsymbol{k}+\boldsymbol{q}}-\phi_{\boldsymbol{k}}\right)\right] V_{q}\left[n_{B}\left(\xi_{\boldsymbol{k}+\boldsymbol{q} \lambda}-\xi_{k}\right)+n_{F}\left(\xi_{\boldsymbol{k}+\boldsymbol{q} \lambda}\right)\right] \\
& \operatorname{Im}\left[\frac{1}{\epsilon\left(q, \xi_{\boldsymbol{k}+\boldsymbol{q} \lambda}-\xi_{k}+i 0^{+}\right)}\right]
\end{aligned}
$$

At zero temperature, it can be seen that interband scattering from the valence band $\lambda=-1$ vanishes in Eq. $(2.21)$, since $n_{B}\left(-\varepsilon_{\boldsymbol{k}+\boldsymbol{q}}-\varepsilon_{k}\right)=-1$ and $n_{F}\left(-\varepsilon_{\boldsymbol{k}+\boldsymbol{q}}-\varepsilon_{F}\right)=1$.

Therefore, only intraband transition $\lambda=1$ contributes to the quasiparticle decay, and Eq. (2.21) becomes (changing the integration variable $\boldsymbol{q} \rightarrow \boldsymbol{k}^{\prime}-\boldsymbol{k}$ ):

$$
\begin{aligned}
\operatorname{Im} \Sigma_{+}^{\mathrm{R}}= & -\frac{e^{2}}{4 \pi} \int_{k_{F}}^{k} \mathrm{~d} k^{\prime} k^{\prime} \int_{0}^{2 \pi} \mathrm{d} \phi_{\boldsymbol{k}^{\prime}}\left(1+\cos \phi_{\boldsymbol{k}^{\prime}}\right) \frac{1}{\sqrt{k^{2}+k^{\prime 2}-2 k k^{\prime} \cos \phi_{\boldsymbol{k}^{\prime}}}} \\
& \operatorname{Im}\left[\frac{1}{\epsilon\left(\left|\boldsymbol{k}-\boldsymbol{k}^{\prime}\right|, \varepsilon_{k^{\prime}}-\varepsilon_{k}+i 0^{+}\right)}\right],
\end{aligned}
$$

where $\phi_{\boldsymbol{k}^{\prime}}$ is the scattering angle from $\boldsymbol{k}$ to $\boldsymbol{k}^{\prime}=\boldsymbol{k}+\boldsymbol{q}$ (with $\phi_{\boldsymbol{k}}=0$ ). In order to maintain analytic tractability, we consider the long-wavelength $x=q / k_{F} \ll 1$ limit and perform an analytical evaluation of the quasiparticle lifetime. For small 
$x$, the dominant contribution in Eq. (2.22) from the imaginary part of the dielectric function comes from low energies $y=\omega / v q \ll 1$, in which case the irreducible polarizability for graphene (in the doped regime) is given in the leading order from Eq. (2.4) by $\Pi^{\mathrm{cc}}(q, \omega) \simeq-\nu\left(\varepsilon_{F}\right)(1+i y)-(q / 4 v)$. The dielectric function in Eq. (2.22) is calculated as $\epsilon(q, \omega)=1-V_{q} \Pi^{\mathrm{cc}}(q, \omega)$, and $\operatorname{Im}[1 / \epsilon(q, \omega)] \simeq-\omega / v q_{\mathrm{TF}}$ up to leading order in $\omega$ (here we have defined the Thomas-Fermi wavenumber as $q_{\mathrm{TF}}=4 e^{2} k_{F} / v$ ). Substituting this expression for the dielectric function in Eq. (2.22), the following expression is obtained:

$$
\operatorname{Im} \Sigma_{+}^{\mathrm{R}}=\frac{e^{2}}{4 \pi q_{\mathrm{TF}}} \int_{k_{F}}^{k} \mathrm{~d} k^{\prime} k^{\prime}\left(k-k^{\prime}\right) \int_{0}^{2 \pi} \mathrm{d} \phi_{\boldsymbol{k}^{\prime}} \frac{1+\cos \phi_{\boldsymbol{k}^{\prime}}}{\sqrt{k^{2}+k^{\prime 2}-2 k k^{\prime} \cos \phi_{\boldsymbol{k}^{\prime}}}}
$$

Carrying out the angular integral, we get

$$
\operatorname{Im} \Sigma_{+}^{\mathrm{R}}=\frac{e^{2}}{4 \pi q_{\mathrm{TF}}} \int_{k_{F}}^{k} \mathrm{~d} k^{\prime} k^{\prime}\left(k^{\prime}-k\right) \frac{2}{k k^{\prime}\left|k-k^{\prime}\right|}\left[\left(k+k^{\prime}\right)^{2} \boldsymbol{K}(-z)-\left(k-k^{\prime}\right)^{2} \boldsymbol{E}(-z)\right],
$$

where $\boldsymbol{K}, \boldsymbol{E}$ are the complete elliptic integrals of the first and second kind, respectively, and $z=4 k k^{\prime} /\left|k-k^{\prime}\right|$. Since $\left|k-k^{\prime}\right| \ll k_{F}, z \gg 1$, we can approximate $\boldsymbol{K}$ and $\boldsymbol{E}$ by their asymptotic expansions: $\boldsymbol{K}(-z) \sim \ln (4 \sqrt{z}) / \sqrt{z}$ and $\boldsymbol{E}(-z) \sim \sqrt{z}$. Eq. (2.24) then becomes

$$
\operatorname{Im} \Sigma_{+}^{\mathrm{R}}=-\frac{e^{2}}{2 \pi k q_{\mathrm{TF}}} \int_{k_{F}}^{k} \mathrm{~d} k^{\prime} \frac{\left|k-k^{\prime}\right|}{2 \sqrt{k k^{\prime}}}\left[\left(k+k^{\prime}\right)^{2} \ln \left(\frac{8 \sqrt{k k^{\prime}}}{\left|k-k^{\prime}\right|}\right)-4 k k^{\prime}\right] .
$$

Let $\delta\left(k^{\prime}\right)=k-k^{\prime} \ll k_{F}$ and change the integration variable from $k^{\prime}$ to $\delta\left(k^{\prime}\right)$ in Eq. (2.21). Expanding the integrand up to leading order in $\delta / k$ and integrating, we 
obtain the following result to leading order in $\delta\left(k_{F}\right) / k_{F}$

$$
\begin{aligned}
\operatorname{Im} \Sigma_{+}^{\mathrm{R}} & =\frac{e^{2}}{\pi q_{\mathrm{TF}}} \int_{0}^{\delta\left(k_{F}\right)} \mathrm{d} \delta \delta\left[\ln \left(\frac{\delta}{8 k}\right)+1\right] \\
& =\frac{e^{2}}{2 \pi q_{\mathrm{TF}}} \delta^{2}\left(k_{F}\right)\left\{\ln \left[\frac{\delta\left(k_{F}\right)}{8 k_{F}}\right]+\frac{1}{2}\right\} .
\end{aligned}
$$

Since $\xi_{k}=v\left(k-k_{F}\right)=v \delta_{F}$, we can rewrite Eq. (2.26) as

$$
\operatorname{Im} \Sigma_{+}^{\mathrm{R}}=\frac{\xi_{k}^{2}}{8 \pi \varepsilon_{F}}\left[\ln \left(\frac{\xi_{k}}{8 \varepsilon_{F}}\right)+\frac{1}{2}\right]
$$

In the following, we calculate the imaginary part of the self-energy for a regular 2DEG with a parabolic spectrum (i.e., $\xi_{k}=k^{2} / 2 m-\mu$ ), and make a comparison with that for graphene presented above. The result for regular 2DEG was first obtained in Ref. [40] but here we provide an alternative derivation. For a 2DEG, the imaginary part of the self-energy is given by [cf. Eq. (2.21)]:

$$
\operatorname{Im} \Sigma_{\boldsymbol{k}}^{\mathrm{R}}\left(\xi_{k}\right)=\sum_{\boldsymbol{q}} V_{q}\left[n_{B}\left(\xi_{\boldsymbol{k}+\boldsymbol{q}}-\xi_{k}\right)+n_{F}\left(\xi_{\boldsymbol{k}+\boldsymbol{q}}\right)\right] \operatorname{Im}\left[\frac{1}{\epsilon\left(q, \xi_{\boldsymbol{k}+\boldsymbol{q}}-\xi_{k}+i 0^{+}\right)}\right]
$$

At zero temperature,

$$
\begin{aligned}
\operatorname{Im} \Sigma^{\mathrm{R}}= & -\frac{e^{2}}{2 \pi} \int_{0}^{2 \pi} \mathrm{d} \phi_{\boldsymbol{k}^{\prime}} \int_{k_{F}}^{k} \mathrm{~d} k^{\prime} k^{\prime} \frac{1}{\sqrt{k^{2}+k^{\prime 2}-2 k k^{\prime} \cos \phi_{\boldsymbol{k}^{\prime}}}} \\
& \operatorname{Im}\left[\frac{1}{\epsilon\left(\left|\boldsymbol{k}-\boldsymbol{k}^{\prime}\right|, \xi_{k^{\prime}}-\xi_{k}+i 0^{+}\right)}\right] .
\end{aligned}
$$

For $x=q / k_{F} \ll 1, y=\omega / v_{F} q \ll 1$ (here $v_{F}$ is the Fermi velocity $v_{F}=k_{F} / m$ ), the irreducible polarizability for a regular $2 \mathrm{DEG}$ is given to leading order by $\Pi^{\mathrm{cc}}(q, \omega) \simeq$ $-\nu(1+i y)$, where $\nu=m / \pi$ is the density of states for the 2DEG. Therefore $\operatorname{Im}[1 / \epsilon(q, \omega)] \simeq-\omega / v_{F} q_{\mathrm{TF}}$ to leading order of $\omega$, with the Thomas-Fermi wavenumber given by $q_{\mathrm{TF}}=2 m e^{2} / \hbar^{2}$. Eq. (2.29) then becomes

$$
\operatorname{Im} \Sigma^{\mathrm{R}}=\frac{e^{2}}{4 \pi m v_{F} q_{\mathrm{TF}}} \int_{k_{F}}^{k} \mathrm{~d} k^{\prime} k^{\prime}\left(k^{\prime 2}-k^{2}\right) \int_{0}^{2 \pi} \mathrm{d} \phi_{\boldsymbol{k}^{\prime}} \frac{1}{\sqrt{k^{2}+k^{\prime 2}-2 k k^{\prime} \cos \phi_{\boldsymbol{k}^{\prime}}}},
$$


Carrying out the angular integral, we get

$$
\operatorname{Im} \Sigma^{\mathrm{R}}=-\frac{1}{2 \pi m k_{F}} \int_{k_{F}}^{k} \mathrm{~d} k^{\prime} k^{\prime}\left(k+k^{\prime}\right) \boldsymbol{K}(-z) .
$$

Using the asymptotic formula $\boldsymbol{K}(-z) \sim \ln (4 \sqrt{z}) / \sqrt{z}$ for $z \gg 1$, Eq. (2.31) becomes

$$
\operatorname{Im} \Sigma^{\mathrm{R}}=-\frac{1}{4 \pi m k_{F}} \int_{k_{F}}^{k} \mathrm{~d} k^{\prime} \sqrt{\frac{k^{\prime}}{k}}\left(k^{2}-k^{\prime 2}\right) \ln \left(\frac{8 \sqrt{k k^{\prime}}}{\left|k-k^{\prime}\right|}\right)
$$

Again, let $\delta\left(k^{\prime}\right)=k-k^{\prime} \ll k_{F}$ and change the integration variable from $k^{\prime}$ to $\delta\left(k^{\prime}\right)$ in Eq. (2.32). Expanding the integrand to leading order in $\delta / k$ and integrating, we obtain the following result to leading order in $\delta\left(k_{F}\right) / k_{F}$,

$$
\begin{aligned}
\operatorname{Im} \Sigma^{\mathrm{R}} & =\frac{1}{2 \pi m} \int_{0}^{\delta\left(k_{F}\right)} \mathrm{d} \delta \delta \ln \left(\frac{\delta}{8 k_{F}}\right) \\
& =\frac{1}{4 \pi m} \delta^{2}\left(k_{F}\right)\left\{\ln \left[\frac{\delta\left(k_{F}\right)}{8 k_{F}}\right]-\frac{1}{2}\right\} .
\end{aligned}
$$

Since $\xi_{k}=\left(k^{2}-k_{F}^{2}\right) / 2 m \simeq k_{F}\left(k-k_{F}\right) / m=k_{F} \delta\left(k_{F}\right) / m$, we can rewrite Eq. (2.33) as

$$
\operatorname{Im} \Sigma^{\mathrm{R}}=\frac{\xi_{k}^{2}}{8 \pi \varepsilon_{F}}\left[\ln \left(\frac{\xi_{k}}{16 \varepsilon_{F}}\right)-\frac{1}{2}\right]
$$

We note that the sign of the subleading term [i.e. the term of $\mathcal{O}\left(\xi_{k}^{2}\right)$ ] in the expression of the quasiparticle decay rate for extrinsic graphene is different from that for a regular 2DEG, which is due to the presence of a chirality factor $\left[1+\cos \left(\phi_{\boldsymbol{k}+\boldsymbol{q}}-\phi_{\boldsymbol{k}}\right)\right] / 2$ in the self-energy expression for graphene.

\subsubsection{Renormalization factor}

Next we consider the renormalization factor for the Dirac quasiparticle $Z=$ $1 /(1-A)$, where $A$ is the derivative of the real part of the self-energy Eq. (2.17) 
with respect to energy at the Fermi level:

$$
A=-\left.\frac{\partial}{\partial \xi} \operatorname{Re} \sum_{\boldsymbol{q}, \lambda} \int_{-\infty}^{\infty} \frac{\mathrm{d} \omega}{2 \pi} G_{\boldsymbol{k}+\boldsymbol{q} \lambda}^{0}\left(i k_{n}+i \omega\right) \frac{1+\lambda \cos \left(\phi_{\boldsymbol{k}+\boldsymbol{q}}-\phi_{\boldsymbol{k}}\right)}{2} \frac{V_{q}}{\epsilon(q, i \omega)}\right|_{k=k_{F}, \xi=0},
$$

where the analytic continuation $i k_{n} \rightarrow \xi+i 0^{+}$applies after performing the integral. In the following, we perform the analytic continuation $i k_{n} \rightarrow \xi+i 0^{+}$before the integrations [34], leading to a decomposition of a line contribution and a pole contribution, and then move the differentiation with respect to $\xi$ inside the integral, thereby obtaining

$$
\begin{aligned}
A= & -\left.\sum_{\boldsymbol{q}, \lambda} \operatorname{Re} \int_{-\infty}^{\infty} \frac{\mathrm{d} \omega}{2 \pi} \frac{\partial}{\partial \xi} G_{\boldsymbol{k}+\boldsymbol{q} \lambda}^{0}(\xi+i \omega) \frac{1+\lambda \cos \left(\phi_{\boldsymbol{k}+\boldsymbol{q}}-\phi_{\boldsymbol{k}}\right)}{2} \frac{V_{q}}{\epsilon(q, i \omega)}\right|_{k=k_{F}, \xi=0} \\
& -\operatorname{Re} \sum_{\boldsymbol{q}, \lambda} \frac{\partial}{\partial \xi}\left\{\left[\theta\left(-\xi_{\boldsymbol{k}+\boldsymbol{q} \lambda}\right)-\theta\left(\xi-\xi_{\boldsymbol{k}+\boldsymbol{q} \lambda}\right)\right]\right. \\
& \left.\frac{1+\lambda \cos \left(\phi_{\boldsymbol{k}+\boldsymbol{q}}-\phi_{\boldsymbol{k}}\right)}{2} \frac{V_{q}}{\epsilon\left(q, \xi_{\boldsymbol{k}+\boldsymbol{q} \lambda}-\xi\right)}\right\}\left.\right|_{k=k_{F}, \xi=0},
\end{aligned}
$$

where the first term corresponds to the line contribution and the second to the pole contribution. The pole contribution can be evaluated as

$$
\begin{aligned}
& -\operatorname{Re} \sum_{\boldsymbol{q}, \lambda} \frac{\partial}{\partial \xi}\left\{\left[\theta\left(-\xi_{\boldsymbol{k}+\boldsymbol{q} \lambda}\right)-\theta\left(\xi-\xi_{\boldsymbol{k}+\boldsymbol{q} \lambda}\right)\right] \frac{1+\lambda \cos \left(\phi_{\boldsymbol{k}+\boldsymbol{q}}-\phi_{\boldsymbol{k}}\right)}{2}\right. \\
& \left.\frac{V_{q}}{\epsilon\left(q, \xi_{\boldsymbol{k}+\boldsymbol{q} \lambda}-\xi\right)}\right\}\left.\right|_{k=k_{F}, \xi=0} \\
= & \left.\frac{1}{2} \sum_{\boldsymbol{q} \lambda} \delta\left(\xi_{\boldsymbol{k}+\boldsymbol{q} \lambda}\right)\left[1+\lambda \cos \left(\phi_{\boldsymbol{k}+\boldsymbol{q}}-\phi_{\boldsymbol{k}}\right)\right] \frac{V_{q}}{\epsilon(q, 0)}\right|_{k=k_{F}},
\end{aligned}
$$


while the line contribution can be integrated by parts and thereby simplified as

$$
\begin{aligned}
& -\left.\sum_{\boldsymbol{q}, \lambda} \operatorname{Re} \int_{-\infty}^{\infty} \frac{\mathrm{d} \omega}{2 \pi} \frac{\partial}{\partial \xi} G_{\boldsymbol{k}+\boldsymbol{q} \lambda}(\xi+i \omega) \frac{1+\lambda \cos \left(\phi_{\boldsymbol{k}+\boldsymbol{q}}-\phi_{\boldsymbol{k}}\right)}{2} \frac{V_{q}}{\epsilon(q, i \omega)}\right|_{k=k_{F}, \xi=0} \\
= & \left.\sum_{\boldsymbol{q}, \lambda} \operatorname{Re} \int_{0}^{\infty} \frac{i \mathrm{~d} \omega}{\pi} \frac{1+\lambda \cos \left(\phi_{\boldsymbol{k}+\boldsymbol{q}}-\phi_{\boldsymbol{k}}\right)}{2} \frac{V_{q}}{\epsilon(q, i \omega)} \frac{\partial}{\partial \omega} G_{\boldsymbol{k}+\boldsymbol{q} \lambda}(i \omega)\right|_{k=k_{F}} \\
= & -\frac{1}{2} \sum_{\boldsymbol{q} \lambda} \delta\left(\xi_{\boldsymbol{k}+\boldsymbol{q} \lambda}\right)\left[1+\lambda \cos \left(\phi_{\boldsymbol{k}+\boldsymbol{q}}-\phi_{\boldsymbol{k}}\right)\right] \frac{V_{q}}{\epsilon(q, 0)} \\
+ & \left.\frac{1}{2 \pi} \operatorname{Im} \sum_{\boldsymbol{q} \lambda} \int_{0}^{\infty} \mathrm{d} \omega G_{\boldsymbol{k}+\boldsymbol{q} \lambda}(i \omega)\left[1+\lambda \cos \left(\phi_{\boldsymbol{k}+\boldsymbol{q}}-\phi_{\boldsymbol{k}}\right)\right] V_{q} \frac{\partial}{\partial \omega} \frac{1}{\epsilon(q, i \omega)}\right|_{k=k_{F}} .(2.3)
\end{aligned}
$$

Combining the line and pole contributions as obtained above, we get

$$
A=\left.\frac{1}{\pi} \operatorname{Im} \sum_{\boldsymbol{q}, \lambda} \int_{0}^{\infty} \mathrm{d} \omega G_{\boldsymbol{k}+\boldsymbol{q} \lambda}(i \omega) V_{q} \frac{1+\lambda \cos \left(\phi_{\boldsymbol{k}+\boldsymbol{q}}-\phi_{\boldsymbol{k}}\right)}{2} \frac{\partial}{\partial \omega} \frac{1}{\epsilon(q, i \omega)}\right|_{k=k_{F}} .
$$

The irreducible polarizability Eq. (2.5) for $x \ll 1$ and $y>1$ is $\Pi(q, \omega) \simeq-\nu(1-$ $\left.y / \sqrt{y^{2}-1}\right)-i(q / 4 v) / \sqrt{y^{2}-1}$. For small $x \ll 1$, we approximate the chirality factor to leading order as $\left(1+\cos \theta_{\boldsymbol{k}^{\prime}, \boldsymbol{k}}\right) / 2 \simeq 1$ and $\left(1-\cos \theta_{\boldsymbol{k}^{\prime}, \boldsymbol{k}}\right) / 2 \simeq\left(q \sin \phi_{k} / 2 k\right)^{2}$. Evaluating Eq. (2.39) up to leading order in $r_{s}$, we obtain the renormalization factor for the Dirac quasiparticle in the $r_{s} \ll 1$ limit as

$$
Z=1 /\left[1+\frac{r_{s}}{\pi}\left(1+\frac{\pi}{2}\right)\right]
$$

In the leading order of $r_{s}$, Eq. (2.40) is similar to the corresponding expression for regular 2DEG [41] $Z=1-\left(r_{s} / 2 \pi\right)(1+\pi / 2)$. Beyond the leading order of $r_{s}$, the $q$-integral in $A$ has a logarithmic divergence due to the interband contribution to the polarizability. Introducing a momentum cutoff $k_{c}=1 / a$ where $a=1.42 \AA$ is the graphene lattice spacing, we find the corresponding logarithmic correction $\sim$ $r_{s}^{2} \ln \left(k_{c} / k_{F}\right)$ appears in the second order term of $r_{s}$ in the denominator of Eq. (2.40). 


\subsubsection{Renormalized velocity}

For quasiparticles with regular quadratic spectrum, it is well-known [34] that electron-electron interaction gives rise to effective mass renormalization of the Fermi liquid. In contrast, as the Dirac quasiparticle in graphene is massless, we have, instead, a renormalization of the quasiparticle velocity. We proceed to calculate the effective velocity renormalization below, which is given by $v / v^{*}=(1-A) /(1+B)$, with $B$ being the derivative of the real part of the retarded self-energy with respect to momentum $k$ and is obtained from Eq. (2.35) by replacing $\partial / \partial \varepsilon \rightarrow(1 / v) \partial / \partial k$ :

$$
B=-\left.\frac{1}{v} \frac{\partial}{\partial k} \operatorname{Re} \sum_{\boldsymbol{q}, \lambda} \int_{-\infty}^{\infty} \frac{\mathrm{d} \omega}{2 \pi} G_{\boldsymbol{k}+\boldsymbol{q} \lambda}^{0}\left(i k_{n}+i \omega\right) \frac{V_{q}}{\epsilon(q, i \omega)} \frac{1+\lambda \cos \left(\phi_{\boldsymbol{k}+\boldsymbol{q}}-\phi_{k}\right)}{2}\right|_{k=k_{F}, \xi=0}
$$

Since the effective velocity at zero temperature in the lowest leading order in $r_{s}$ is only due to the contribution from static dielectric response [41], we can evaluate $v^{*} / v$ with the dielectric function in the static limit $\epsilon(q, \omega)=\epsilon(q, 0)$. It then follows from Eq. (2.39) that $A=0$, and

$$
\begin{aligned}
B= & \frac{1}{\pi v} \operatorname{Im} \sum_{\boldsymbol{q}, \lambda} \lambda \frac{\partial \xi_{\boldsymbol{k}+\boldsymbol{q}}}{\partial k} \frac{V_{q}}{\epsilon(q, 0)} \frac{1+\lambda \cos \left(\phi_{\boldsymbol{k}+\boldsymbol{q}}-\phi_{\boldsymbol{k}}\right)}{2} \int_{0}^{\infty} \mathrm{d} \omega \frac{\partial}{\partial \omega} G_{\boldsymbol{k}+\boldsymbol{q} \lambda}(i \omega) \\
& -\frac{1}{\pi v} \operatorname{Re} \sum_{\boldsymbol{q}, \lambda} \frac{V_{q}}{\epsilon(q, 0)} \frac{\partial}{\partial k} \frac{1+\lambda \cos \left(\phi_{\boldsymbol{k}+\boldsymbol{q}}-\phi_{\boldsymbol{k}}\right)}{2} \int_{0}^{\infty} \mathrm{d} \omega G_{\boldsymbol{k}+\boldsymbol{q} \lambda}(i \omega)
\end{aligned}
$$

At $k=k_{F}$, the first term yields

$$
\begin{aligned}
& \left.\frac{1}{\pi v} \operatorname{Im} \sum_{\boldsymbol{q}, \lambda} \lambda \frac{\partial \xi_{\boldsymbol{k}+\boldsymbol{q}}}{\partial k} \frac{V_{q}}{\epsilon(q, 0)} \frac{1+\lambda \cos \left(\phi_{\boldsymbol{k}+\boldsymbol{q}}-\phi_{\boldsymbol{k}}\right)}{2} G_{\boldsymbol{k}+\boldsymbol{q} \lambda}(i \omega)\right|_{0} ^{\infty} \\
= & \sum_{\boldsymbol{q}} \delta\left(\varepsilon_{F}-v|\boldsymbol{k}+\boldsymbol{q}|\right) \frac{V_{q}}{\epsilon(q, 0)} \frac{1+\lambda \cos \left(\phi_{\boldsymbol{k}+\boldsymbol{q}}-\phi_{\boldsymbol{k}}\right)}{2} \frac{k+q \cos \phi_{k}}{|\boldsymbol{k}+\boldsymbol{q}|} \\
\simeq & -\frac{r_{s}}{\pi}\left(\frac{5}{3}+\ln r_{s}\right)
\end{aligned}
$$


where we arrived at the last step by evaluating the angular integral, expanding in leading order of $r_{s}$, and then evaluating the resultant $q$ integral. The second term in Eq. (2.42) yields

$$
\begin{aligned}
& -\frac{1}{2 \pi v} \operatorname{Re} \sum_{\boldsymbol{q}, \lambda} \lambda \frac{V_{q}}{\epsilon(q, 0)} \frac{\partial \cos \left(\phi_{\boldsymbol{k}+\boldsymbol{q}}-\phi_{\boldsymbol{k}}\right)}{\partial k} \int_{0}^{\infty} \mathrm{d} \omega G_{\boldsymbol{k}+\boldsymbol{q} \lambda}(i \omega) \\
= & \frac{1}{2 v} \operatorname{Re} \sum_{\boldsymbol{q}} \lambda \frac{V_{q}}{\epsilon(q, 0)} \frac{\partial \cos \left(\phi_{\boldsymbol{k}+\boldsymbol{q}}-\phi_{\boldsymbol{k}}\right)}{\partial k} \theta\left(v|\boldsymbol{k}+\boldsymbol{q}|-\varepsilon_{F}\right) \\
\sim & \frac{r_{s}}{4} \ln \left(\frac{k_{c}}{k_{F}}\right),
\end{aligned}
$$

within logarithmic accuracy, where we have arrived at the last step by keeping only the most dominant (i.e., divergent) contribution of the $q$ integral and have introduced a cutoff momentum $k_{c}=1 / a(a=2.46 \AA$ is the graphene lattice spacing $)$ to regularize the divergent contribution. Combining Eqs. (2.43)-(2.44), we obtain the renormalized velocity at the Fermi level up to leading order of $r_{s}$ and within logarithmic accuracy

$$
\frac{v^{*}}{v}=1-\frac{r_{s}}{\pi}\left[\frac{5}{3}+\ln \left(r_{s}\right)\right]+\frac{r_{s}}{4} \ln \left(\frac{k_{c}}{k_{F}}\right) .
$$

Here, we note that the first two terms in Eq. (2.45) derive from the intraband contribution and are similar to the expression for the regular 2DEG [42] $\mathrm{m}^{*} / \mathrm{m}=$ $1+\left(r_{s} / 2 \pi\right)\left[2+\ln \left(r_{s} / 4\right)\right]$ whereas the last term arises solely from the interband contribution. The velocity renormalization is in general a function of $k$, and in addition to $v^{*}$ at the Fermi level, we have evaluated $v^{*}$ at the Dirac point $k=0$ :

$$
\frac{v^{*}}{v}=1-r_{s}\left\{1+\frac{1}{4} \ln \left[\frac{1+4 r_{s}}{4 r_{s}}\right]-\frac{1}{4} \ln \left(\frac{k_{c}}{k_{F}}\right)\right\}
$$

From Eqs. (2.45)-(2.46), we see that the linear single-particle band dispersion of graphene is renormalized by many-body effects from Coulomb interaction to a band 
dispersion which is nonlinear.

\subsection{Intrinsic graphene}

\subsubsection{Quasiparticle decay rate}

We now calculate the imaginary part of the quasiparticle self-energy for intrinsic graphene (i.e. $\varepsilon_{F}=0$ ). From Eq. (2.17), the intraband contribution $\lambda=1$ vanishes at zero temperature. This makes physical sense because all the electronic states in the valence band are fully occupied, rendering intraband transition within the valence band impossible. The only possible type of transition that can happen for an electron is interband transition, with a contribution to the imaginary part of the self-energy given by

$$
\begin{aligned}
\operatorname{Im} \Sigma_{k \pm}^{\mathrm{R}}(\omega)= & -\frac{e^{2}}{4 \pi} \int_{0}^{\infty} \mathrm{d} q \int_{0}^{2 \pi} \mathrm{d} \phi_{\boldsymbol{q}}\left[1 \pm \cos \left(\phi_{\boldsymbol{k}+\boldsymbol{q}}-\phi_{\boldsymbol{k}}\right)\right] \theta\left(\omega-\varepsilon_{\boldsymbol{k}+\boldsymbol{q}}\right) \\
& \operatorname{Im}\left[\frac{1}{\epsilon\left(q, \varepsilon_{\boldsymbol{k}+\boldsymbol{q}}-\omega\right)}\right] .
\end{aligned}
$$

The irreducible polarizability for intrinsic graphene is given by $\Pi_{-}^{\mathrm{cc}}(q, \omega)$ in Eq. (2.14). Since $\operatorname{Im}\left[1 / \epsilon\left(q, \varepsilon_{\boldsymbol{k}+\boldsymbol{q}}-\omega\right)\right] \sim \theta\left(\left|\varepsilon_{\boldsymbol{k}+\boldsymbol{q}}-\omega\right|-v q\right)$, the on-shell quasiparticle lifetime $\operatorname{Im} \Sigma_{\boldsymbol{k}+}^{\mathrm{R}}\left(\xi_{k}\right) \sim \sum_{\boldsymbol{q}} \theta\left(\left|\varepsilon_{\boldsymbol{k}+\boldsymbol{q}}-\varepsilon_{k}\right|-\varepsilon_{q}\right) \theta\left(\varepsilon_{k}-\varepsilon_{\boldsymbol{k}+\boldsymbol{q}}\right)$ vanishes identically because of phase space restrictions imposed by the $\theta$-functions. In the following, we obtain an approximate analytical result of the imaginary part of the self-energy by expanding $q$ up to leading order inside the $q$ integral. For interband transitions in intrinsic graphene, we expand the dielectric function $\epsilon(q, \omega)=1-V(q) \Pi_{-}^{\mathrm{cc}}$ [where $\Pi_{-}^{\mathrm{cc}}$ is 
given by Eq. (2.14)] in the regime $y=\omega / v q \gg 1$ up to leading order of $1 / y$, obtaining $\epsilon(q, \omega) \simeq 1+i \pi r_{s} / 4 y$ and hence $\operatorname{Im}[1 / \epsilon(q, \omega)]=-\pi r_{s} \theta(\omega-v q) / 4 y$. Eq. (2.47) at the Dirac point $k=0$ becomes

$$
\begin{aligned}
\operatorname{Im} \Sigma_{k=0 \pm}^{\mathrm{R}}(\omega) & \simeq \frac{2 \pi r_{s} e^{2}}{16} \int_{0}^{\infty} \mathrm{d} q \int_{0}^{\omega / v} \frac{q}{v q-\omega} \\
& =\frac{\pi r_{s} e^{2}}{8}(1-2 \ln 2) \omega
\end{aligned}
$$

In fact, an exact analytical result can be obtained for $\operatorname{Im} \Sigma_{k=0}^{\mathrm{R}}(\omega)$ from Eq. (2.47) [28] and is given by

$$
\operatorname{Im} \Sigma_{k=0 \pm}^{\mathrm{R}}(\omega)=\omega f\left(r_{s}\right)
$$

where

$$
f\left(r_{s}\right)=\frac{2}{\pi^{2} r_{s}}\left[\pi\left(1-r_{s}\right)+\frac{8-\left(\pi r_{s}\right)^{2}}{4 \sqrt{\left(\pi r_{s}\right)^{2}-4}} \ln \frac{\pi r_{s}-\sqrt{\left(\pi r_{s}\right)^{2}-4}}{\pi r_{s}+\sqrt{\left(\pi r_{s}\right)^{2}-4}}\right]
$$

This linear relation is exact for all $\omega$, indicating that intrinsic graphene is a marginal Fermi liquid. We note that there is no plasmon contribution to the imaginary part of the self-energy for intrinsic graphene. The contribution of the interband electron-hole excitations gives rise to the linear behavior of $\operatorname{Im} \Sigma_{k=0 \pm}^{\mathrm{R}}$. However, for doped graphene the contribution of the interband electron-hole excitations is completely suppressed due to phase space restrictions at zero temperature. The contribution of the intraband virtual single-particle excitations and/or the virtual excitations of plasmons give rise to higher powers of $\omega\left(\right.$ i.e. $\left.\omega^{2}\right)$ in the imaginary part of the self-energy in the doped case, which restores the usual Fermi liquid behavior. Thus, the qualitative difference between intrinsic $(\operatorname{Im} \Sigma \sim \omega))$ and extrinsic $\left(\operatorname{Im} \Sigma \sim \omega^{2}\right)$ graphene can be completely understood by noting that the intrinsic 
system is an insulator (albeit a zero-gap semiconductor with no intraband singleparticle excitation) and the extrinsic case has a Fermi surface with intraband singleparticle excitations. Thus, any doping of graphene (intentional or unintentional) will immediately suppress its marginal Fermi liquid intrinsic character, converting it to a regular 2D Fermi liquid.

\subsubsection{Renormalization factor}

The renormalization factor can be obtained from Eq. (2.35), from which we find that $A \sim \int_{0} \mathrm{~d} q / q$ diverges logarithmically, which is due to the unscreened nature of the Coulomb potential (the same divergence occurs for the exchange energy of a regular 2DEG). Therefore the renormalization factor $Z=0$, showing that as doping goes to zero, the magnitude of the step at the Fermi energy $\varepsilon=0$ also shrinks to zero, approaching the Dirac point, where the notion of a "Fermi surface" no longer applies, the quintessential behavior of a marginal Fermi liquid. Using the Kramers-Kronig relations and from the fact that $\operatorname{Im} \Sigma_{k=0 \pm}^{\mathrm{R}}(\omega) \sim \omega$, we have $\operatorname{Re} \sum_{k=0 \pm}^{\mathrm{R}}(\omega) \sim \omega \ln \omega$, and the renormalization factor $Z \sim 1 / \ln \omega$, which approaches zero logarithmically as $\omega \rightarrow 0$ at the Fermi energy. The spectral function is defined as

$$
\begin{aligned}
A_{\boldsymbol{k} \pm}(\omega) & \equiv-2 \operatorname{Im} G_{k \pm}(\omega) \\
& =\frac{\operatorname{Im} \Sigma_{k \pm}^{\mathrm{R}}(\omega)}{\left[\operatorname{Im} \Sigma_{k \pm}^{\mathrm{R}}(\omega)\right]^{2}+\left[\omega-\operatorname{Re} \Sigma_{k \pm}^{\mathrm{R}}(\omega)\right]^{2}}
\end{aligned}
$$

where $G_{\boldsymbol{k} \pm}(\omega)=1 /\left[\omega-\xi_{k \pm}-\tilde{\Sigma}_{\boldsymbol{k} \pm+}(\omega)\right]$ is the interacting electron Green function with self-energy correction. At $k=0$, the spectral function $A_{k=0}(\omega)=\operatorname{Im} \sum_{k=0 \pm}^{\mathrm{R}}(\omega)$ $/\left\{\left[\operatorname{Im} \sum_{k=0 \pm}^{\mathrm{R}}(\omega)\right]^{2}+\left[\omega-\operatorname{Re} \sum_{k=0 \pm}^{\mathrm{R}}(\omega)\right]^{2}\right\}$ therefore diverges as $A_{k=0}(\omega) \sim 1 / \omega(\ln \omega)^{2}$. 


\subsubsection{Renormalized velocity}

Similarly to the extrinsic case, the effective velocity up to leading order of $r_{s}$ follows from taking the derivative of the real part of the self-energy [with a static dielectric function $\epsilon(q, 0)]$ with respect to $k$ as

$$
\frac{v^{*}}{v}=1+\left(\frac{r_{s}^{*}}{4}\right) \ln \left(\frac{k_{c}}{k_{F}}\right),
$$

here $r_{s}^{*}=r_{s} /\left(1+\pi r_{s} / 2\right)$ is the renormalized interaction parameter. Therefore, it follows from Eq. (2.52) that $v^{*} / v$ diverges for the intrinsic case as doping $k_{F} \rightarrow 0$. These results for the intrinsic graphene are consistent with Ref. [43], where the renormalization group approach is used to arrive at a similar conclusion. In passing, we note that the case for a purely undoped 3D system with a gapless linear energy dispersion was considered in Ref. [44], and was found to exhibit marginal Fermi liquid behavior with a logarithmic energy dependence in $\operatorname{Re} \Sigma$ comparable to the results for intrinsic graphene.

Concluding this chapter, we have developed a theory for the quasiparticle selfenergy due to dynamically-screened Coulomb interaction in graphene from which we calculated the renormalized quasiparticle parameters for both extrinsic and intrinsic graphene. We find that for extrinsic graphene the analytical results for the quasiparticle lifetime, renormalization factor and effective velocity show no deviation from the usual Fermi liquid behavior, and the Fermi liquid description is robust. On the other hand, with precisely zero doping, intrinsic graphene exhibits a quasiparticle decay rate linear in the excitation energy and a zero renormalization factor, indicating that the Fermi liquid description is marginal at the Dirac point. With 
a finite Fermi energy in the extrinsic graphene, the interband single-particle excitations which give rise to the linear $\omega$-dependence of the quasiparticle decay rate (and hence the marginal Fermi liquid behavior) in the intrinsic graphene are suppressed, bringing the system back to a usual Fermi liquid. Since some finite doping is invariable in real systems, real 2D graphene is generically a Fermi liquid. 


\section{Chapter 3}

\section{Many-body effect in graphene}

\section{transport - Coulomb drag}

\subsection{The phenomenon of Coulomb drag}

While electronic structure experiments such as ARPES have revealed detailed subtle many-body effects on the graphene energy spectrum, transport experiments have also revealed some apparently unusual features of graphene transport properties, most noticably that the conductivity has a non-zero minimum value forming a plateau around zero bias gate voltage. Up to now, the transport experiments performed have been focused only on the longitudinal and Hall transport properties, where all of these phenomena depend only on the physics of scattering of individual electrons from impurities with electron-electron many-body interaction effect playing the role of a small quantitative correction. In two-dimensional electron gas (2DEG) semiconductor bilayer structures (e.g. modulation-doped GaAs/ $\mathrm{Al}_{x} \mathrm{Ga}_{1-x} \mathrm{As}$ double quantum wells), electron-electron scattering between the 2DEG layers gives 
rise to the effect of Coulomb drag [45, 46, 47], where a "drag" current is induced purely from the momentum exchanges through interlayer electron-electron scattering events. One injects a current into one of the layer, called the "active layer", in which electrons are driven to flow along the layer. Through Coulomb interaction mediated across the layer, electrons in another layer, called the "passive layer", are also "dragged along" with the electrons in the active layer and thus flow in the same direction. One measures the effect of Coulomb drag by the drag resistivity $\rho_{\mathrm{D}}$, which is defined by the induced drag electric field in the open-circuited passive layer per unit applied current density in the active layer (Fig. 3.1), $\rho_{\mathrm{D}}=E_{\text {passive }} / J_{\text {active }}$. In

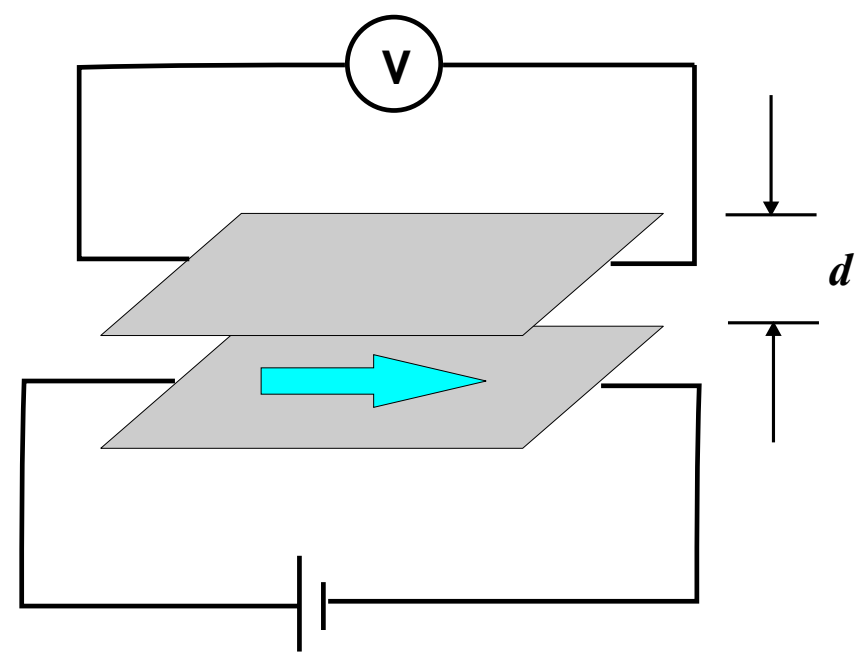

Figure 3.1: The experimental setting of a Coulomb drag experiment. A current is sent through the "active layer" driving electrons to flow along the layer, this "drags along" the electrons to move in the same direction in the open-circuited "passive layer" via interlayer Coulomb interaction, which then induce a voltage that can be measured.

the experimental geometry, this is equal to $\rho_{\mathrm{D}}=(W / L)\left(V_{\text {passive }} / I_{\text {active }}\right)$, where $W$ and $L$ are the width and length of the layers. In high-mobility samples where the disorder is weak, $\rho_{\mathrm{D}}$ goes as $T^{2}$ at low temperatures $T$, and as $1 / d^{4}$ for large bilayer separation $d$ (Refs. [48, 45]). 
In this chapter, we investigate the Coulomb drag in graphene "bilayer" systems with no interlayer tunneling, considering both the intrinsic (chemical potential $\mu=0)$ and extrinsic $(\mu \neq 0)$ cases. We emphasize right in the beginning, so that there is no semantic confusion, what we mean by the terminology 'bilayer' graphene. Our 'bilayer' graphene is two isolated parallel 2D graphene monolayers separated by a distance $d$, with no interlayer tunneling. When $d \gg a$, the electronic structure of each graphene monolayer is unaffected by having the other layer. Each graphene layer is assumed to have its own variable carrier density in the extrinsic case. Our system is thus different from the ordinary bilayer graphene where the two monolayers are separated by the distance $d \sim 3.4 \AA$ with strong interlayer tunneling. Throughout this chapter, we shall also use the terms "undoped" and "doped" interchangeably with "intrinsic" and "extrinsic" respectively; keeping in mind that in experiments the chemical potential can be changed by both chemical doping and gating with an applied voltage. The Coulomb drag in graphene is interesting not only because it is a novel material with a linear energy spectrum, but also because it only spans a thickness of a single carbon atom, the electrons are much more confined along the perpendicular direction compared with 2DEG in a quantum well, where the finite-width thickness has to be taken into account in any quantitative comparison with experiments. Thus, the Coulomb drag phenomenon in graphene is expected to be theoretically very well accounted for with two zero-thickness graphene sheets. In addition, tunneling is only appreciable when the out-of-plane $\pi$ orbitals from the two graphene sheets start to overlap with each other at an interlayer distance $d$ of a few angstroms ( $d \simeq 3.5 \AA$ in naturally occuring graphite), making it possible to 


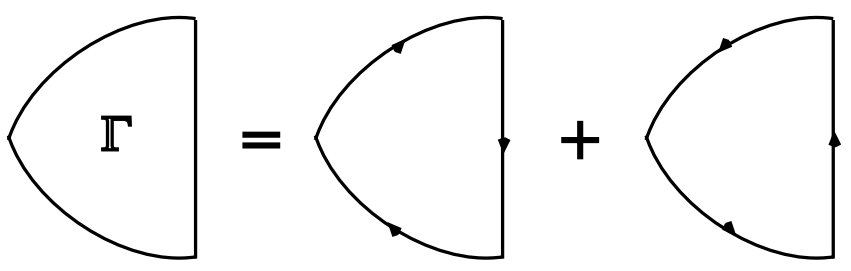

Figure 3.2: Diagram for the nonlinear susceptibility $\Gamma$, which is a three-point vertex function.

study the effect of Coulomb drag for an interlayer separation $d$ down to a few tens of angstroms, about an order of magnitude smaller than is possible in the usual double quantum well systems without appreciable tunneling.

\subsection{Formalism}

In the linear response formalism, we calculate the drag conductivity which is given by $\sigma_{\mathrm{D}}=1 / \rho_{\mathrm{D}}=J_{\text {active }} / E_{\text {passive }}$ from the diagrammatic perturbation theory.

\subsubsection{Drag conductivity}

The central quantity in the Coulomb drag problem is the nonlinear susceptibility $[46,47,49]$, $\Gamma$, given in Fig. 3.3 by the three-point vertex diagram as:

$$
\begin{aligned}
\boldsymbol{\Gamma}(\boldsymbol{q}, \omega)= & \int \frac{\mathrm{d} \varepsilon}{2 \pi i}\left[n_{F}(\varepsilon+\omega)-n_{F}(\varepsilon)\right] \sum_{k} \operatorname{tr}\left\{\left[\tilde{G}_{\boldsymbol{k}-\boldsymbol{q}}^{A}(\varepsilon)-\tilde{G}_{\boldsymbol{k}-\boldsymbol{q}}^{R}(\varepsilon)\right]\right. \\
& \left.\tilde{G}_{\boldsymbol{k}}^{A}(\varepsilon+\omega) \tilde{\boldsymbol{J}}(\boldsymbol{k}) \tilde{G}_{\boldsymbol{k}}^{R}(\varepsilon+\omega)\right\}+\{\boldsymbol{q}, \omega \rightarrow-\boldsymbol{q},-\omega\},
\end{aligned}
$$

where $\tilde{G}_{\boldsymbol{k}}^{R, A}(\varepsilon)=\left(\varepsilon-H_{0} \pm i / 2 \tau\right)^{-1}$ denotes, within the Born approximation for the self-energy, the impurity-averaged retarded/advanced Green function in the sublattice basis, $\tau$ the lifetime due to impurity scattering, $\boldsymbol{J}$ the charge current vertex in the sublattice space, $n_{F}$ the Fermi function and 'tr' the trace. In the rest of this 
chapter, we shall simply denote the $x$-components of $\boldsymbol{\Gamma}$ and $\tilde{\boldsymbol{J}}$ as $\Gamma$ and $\tilde{J}$. The drag conductivity, diagrammatically shown in Fig. 3.3, is given by [46, 47, 49]
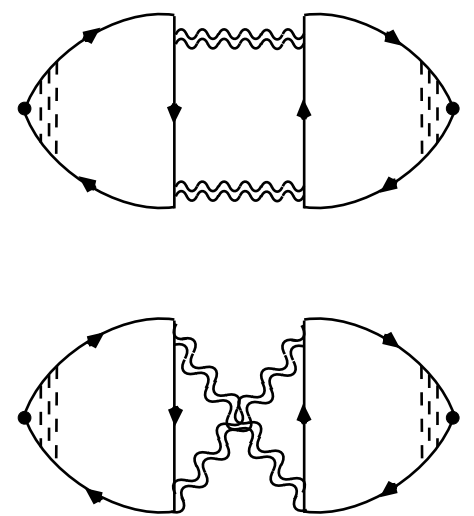

Figure 3.3: Diagrams contributing to the drag resistivity Eq. (3.2). The double wavy lines represent the screened interlayer Coulomb potential Eq. (3.4) and the vertices on the left and on the right denote charge current in the two layers. Dashed vertical lines next to the vertices denote impurity vertex correction to the charge current.

$$
\sigma_{\mathrm{D}}=\frac{1}{16 \pi k_{B} T} \sum_{q} \int_{0}^{\infty} \frac{\mathrm{d} \omega}{\sinh ^{2}\left(\hbar \omega / 2 k_{B} T\right)} \Gamma_{1}(q, \omega) \Gamma_{2}(q, \omega)\left|U_{12}(q, \omega)\right|^{2}
$$

here subscripts ' 1 ' and ' 2 ' are the labels for the two single-layer graphene, $U_{12}$ is the screened interlayer potential, which in the random phase approximation (RPA) is given by

$$
U_{12}(q, \omega)=\frac{V(q) e^{-q d}}{\left[1-\Pi_{1}^{\mathrm{cc}} V(q)\right]\left[1-\Pi_{2}^{\mathrm{cc}} V(q)\right]-\Pi_{1}^{\mathrm{cc}} \Pi_{2}^{\mathrm{cc}} V^{2}(q) e^{-2 q d}}
$$

where $d$ is the interlayer spacing, $V(q)=2 \pi e^{2} / q$ is the bare Coulomb potential, $\Pi_{i}^{c c}(q, \omega)$ is the graphene polarizability [Eq. (2.5) and Eq. (2.14) in Chapter 2] of layer i. Instead of the drag conductivity, in experiments one measures the drag 
resistivity $\rho_{\mathrm{D}}=1 / \sigma_{\mathrm{D}}$, which is given by

$$
\begin{aligned}
\rho_{\mathrm{D}} & =\sigma_{\mathrm{D}}^{-1}=\left[\begin{array}{cc}
\sigma_{11} & \sigma_{12} \\
\sigma_{21} & \sigma_{22}
\end{array}\right]^{-1} \\
& =\frac{1}{\sigma_{11} \sigma_{22}-\sigma_{12} \sigma_{21}}\left[\begin{array}{cc}
\sigma_{22} & -\sigma_{12} \\
-\sigma_{21} & \sigma_{11}
\end{array}\right] .
\end{aligned}
$$

The drag resistivity is then obtained as $\rho_{\mathrm{D}}=\sigma_{\mathrm{D}} /\left(\sigma_{\mathrm{L} 1} \sigma_{\mathrm{L} 2}-\sigma_{\mathrm{D}}^{2}\right) \simeq \sigma_{\mathrm{D}} / \sigma_{\mathrm{L} 1} \sigma_{\mathrm{L} 2}$ since $\sigma_{\mathrm{D}} \ll \sigma_{\mathrm{L} 1}, \sigma_{\mathrm{L} 2}$, where $\sigma_{\mathrm{L} 1,2} \equiv \sigma_{11,22}$ is the longitudinal conductivity of the individual layer 1 or 2 .

We shall restrict ourselves to the Boltzmann regime $(\omega \tau \gg 1$ or $q l \gg 1$, where $l=v \tau$ is the mean free path) corresponding to weak impurity scattering, which is the case relevant to actual experimental situations where high-mobility samples with dilute impurities are used. The longitudinal current for the graphene Hamiltonian in the sublattice space is $\tilde{\boldsymbol{J}}=e \partial H_{0} / \partial \boldsymbol{k}=e v \hat{\boldsymbol{\sigma}}$. In the presence of impurities, vertex correction to the current is taken into account within the impurity ladder approximation, which gives the impurity-dressed current vertex as $\tilde{\boldsymbol{J}}=\left(\tau_{\mathrm{tr}} / \tau\right) e v \hat{\boldsymbol{\sigma}}$, where the transport time $\tau_{\text {tr }}$ for graphene is given by

$$
\tau_{\mathrm{tr}}^{-1}=\left.\pi \sum_{k^{\prime}} n_{i}\left|u_{i}\left(\boldsymbol{k}-\boldsymbol{k}^{\prime}\right)\right|^{2}\left[1-\cos ^{2}\left(\phi_{\boldsymbol{k}^{\prime}}-\phi_{\boldsymbol{k}}\right)\right] \delta\left(\xi_{\boldsymbol{k}+}-\xi_{\boldsymbol{k}^{\prime}+}\right)\right|_{k=k_{F}}
$$

with $n_{i}$ and $u_{i}$ being respectively the impurity density and impurity potential, $\phi_{\boldsymbol{k}}$ is the azithmuthal angle of the momentum $\boldsymbol{k}$, and $\boldsymbol{k}^{\prime}=\boldsymbol{k}+\boldsymbol{q}$. We have given Eq. (3.5) for the transport time here only for the purpose of completeness, as our final results for the drag resistivity do not depend on $\tau_{\mathrm{tr}}$. 


\subsubsection{Nonlinear susceptibility}

In the following, we derive the expression for the nonlinear susceptibility by transforming the Green functions and the current vertices inside the trace of Eq. (3.1) from the sublattice basis to the chiral basis (i.e., the diagonal basis of the Hamiltonian $\left.H_{0}\right)$ :

$$
\begin{aligned}
\boldsymbol{\Gamma}(\boldsymbol{q}, \omega)= & \int \frac{\mathrm{d} \varepsilon}{2 \pi i}\left[n_{F}(\varepsilon+\omega)-n_{F}(\varepsilon)\right] \sum_{k} \operatorname{tr}\left\{U_{\boldsymbol{k}, \boldsymbol{k}-\boldsymbol{q}}\left[G_{\boldsymbol{k}-\boldsymbol{q}}^{A}(\varepsilon)-G_{\boldsymbol{k}-\boldsymbol{q}}^{R}(\varepsilon)\right] U_{\boldsymbol{k}, \boldsymbol{k}-\boldsymbol{q}}^{\dagger}\right. \\
& \left.G_{\boldsymbol{k}}^{A}(\varepsilon+\omega) \boldsymbol{J}(\boldsymbol{k}) G_{\boldsymbol{k}}^{R}(\varepsilon+\omega)\right\}+\{\boldsymbol{q}, \omega \rightarrow-\boldsymbol{q},-\omega\}
\end{aligned}
$$

where $U_{\boldsymbol{k}, \boldsymbol{k}-\boldsymbol{q}}=U_{\boldsymbol{k}}^{\dagger} U_{\boldsymbol{k}-\boldsymbol{q}}$, and $U_{\boldsymbol{k}}$ is the transformation that diagonalizes the graphene Hamiltonian Eq. (1.13),

$$
\begin{aligned}
G_{k}^{R, A}(\varepsilon) & =U_{\boldsymbol{k}}^{\dagger} \tilde{G}_{k}^{R, A}(\varepsilon) U_{\boldsymbol{k}} \\
& =\left[\begin{array}{cc}
1 /\left(\varepsilon-\xi_{k+} \pm i / 2 \tau\right) & 0 \\
0 & 1 /\left(\varepsilon-\xi_{k-} \pm i / 2 \tau\right)
\end{array}\right],
\end{aligned}
$$

is the impurity averaged Green function in the chiral basis, and

$$
\begin{aligned}
J_{k} & =U_{k}^{\dagger} \tilde{J}_{k} U_{k} \\
& =\frac{e v}{2} \frac{\tau_{\mathrm{tr}}}{\tau}\left[\begin{array}{cc}
\cos \phi_{\boldsymbol{k}} & -i \sin \phi_{\boldsymbol{k}} \\
i \sin \phi_{\boldsymbol{k}} & -\cos \phi_{\boldsymbol{k}}
\end{array}\right],
\end{aligned}
$$

is the current vertex operator in the chiral basis. After evaluating the matrix products in Eq. (3.6), taking the trace, and considering the weak scattering (i.e., Boltzmann) limit $\omega \tau \gg 1[49]$, we obtain

$$
\begin{aligned}
\Gamma(\boldsymbol{q}, \omega)= & \tau \sum_{\lambda, \lambda^{\prime}= \pm} \sum_{k}\left[J_{\lambda \lambda}(\boldsymbol{k}+\boldsymbol{q})-J_{\lambda^{\prime} \lambda^{\prime}}(\boldsymbol{k})\right] \\
& \operatorname{Im}\left\{\left[1+\lambda \lambda^{\prime} \cos \left(\phi_{\boldsymbol{k}+\boldsymbol{q}}-\phi_{\boldsymbol{k}}\right)\right] \frac{n_{F}\left(\xi_{\boldsymbol{k} \lambda^{\prime}}\right)-n_{F}\left(\xi_{\boldsymbol{k}+\boldsymbol{q} \lambda}\right)}{\omega+\xi_{\boldsymbol{k} \lambda^{\prime}}-\xi_{\boldsymbol{k}+\boldsymbol{q} \lambda}+i 0^{+}}\right\} .
\end{aligned}
$$


Eq. (3.9) is different from the nonlinear susceptibility for regular 2DEG with quadratic spectrum in two ways: (1) there are contributions to the electron-hole excitations coming from both intraband transitions $\left(\lambda=\lambda^{\prime}\right)$ and interband transitions $\left(\lambda \neq \lambda^{\prime}\right)$;

(2) there is an additional factor $\left[1 \pm \cos \left(\phi_{\boldsymbol{k}+\boldsymbol{q}}-\phi_{\boldsymbol{k}}\right)\right] / 2$, which derives from the chirality of the graphene band structure. Furthermore, the nonlinear susceptibility Eq. (3.9) is not directly proportional to the imaginary part of the polarizability as in regular 2DEG, because here the current $\boldsymbol{J}(\boldsymbol{k})$ is not directly proportional to the momentum $\boldsymbol{k}$ because of the linearity of the energy dispersion. Eq. (3.9) has the same formal structure as in the case of a regular 2DEG with Rashba/Dresselhaus spin-orbit coupling [49], where $\lambda= \pm 1$ describes the two spin-split bands. The finite off-diagonal components of $J$ drop out from the expression of the nonlinear susceptibility, and only the diagonal components $J_{\lambda \lambda}=\lambda\left(\tau_{\text {tr }} / \tau\right) e v \cos \phi_{\boldsymbol{k}}$ enter into the expression Eq. (3.9), corresponding to electrons moving in the conduction band $(\lambda=1)$ with a velocity of constant magnitude $\left(\tau_{\text {tr }} / \tau\right) \boldsymbol{v}$ and valence band $(\lambda=-1)$ with $-\left(\tau_{\text {tr }} / \tau\right) \boldsymbol{v}$. In the following, we consider the Coulomb drag between intrinsic graphene layers and extrinsic graphene layers separately.

\subsection{Drag in intrinsic graphene systems}

For the case where the graphene layers are undoped $\mu=0$, we first state the main result: the drag conductivity between two intrinsic graphene layers, or between one extrinsic and one intrinsic graphene layers, is identically zero. This is not at first sight a trivial consequence of zero doping if one recalls there is a finite conductivity 
(so-called the "minimum conductivity") at zero doping in graphene. A physical explanation and a general argument for the reason why this is so is in order. When the Fermi level is at the Dirac point, the only process for electron-hole pair creation will be interband electron excitation from the valence band to the conduction band by which equal numbers of electrons and holes are created. In the mechanism of Coulomb drag, the applied electric field drives the electrons (or holes) in the active layer in the, say, positive (negative) direction; through Coulomb scattering, momentum is transferred to the passive layer, which drives the carriers (regardless of whether these are electrons or holes) in the same direction as the momentum transfer. In doped systems where there is only one type of carrier (either electron or hole), this gives a finite drag current in the passive layer. Now, in undoped systems where a perfect electron-hole symmetry exists, there are two cases for consideration: (1) If the active layer is undoped, equal numbers of electrons and holes in the active layer will be driven in the opposite direction by the applied electric field, and the net momentum transfer is thus zero. There will be no drag regardless of what the passive layer is. (2) If the active layer is doped while the passive layer is undoped, equal numbers of electrons and holes in the passive layer will be driven in the same direction by the momentum transfer, therefore resulting in a vanishing drag current. The conclusion of these qualitative considerations amounts to a vanishing nonlinear susceptibility $\Gamma(q, \omega)=0$, which we now prove as follows. We first make a change of the integration variable $k^{\prime}=-k$ in Eq. (3.9), and use time-reversal symmetry to 
obtain

$$
\begin{aligned}
\Gamma(\boldsymbol{q}, \omega)= & \tau \sum_{\lambda, \lambda^{\prime}= \pm} \sum_{k^{\prime}}\left[-J_{\lambda \lambda}\left(\boldsymbol{k}^{\prime}-\boldsymbol{q}\right)+J_{\lambda^{\prime} \lambda^{\prime}}\left(\boldsymbol{k}^{\prime}\right)\right] \operatorname{Im}\left\{\left[1+\lambda \lambda^{\prime} \cos \left(\phi_{\boldsymbol{k}}-\phi_{\boldsymbol{k}-\boldsymbol{q}}\right)\right]\right. \\
& \left.\frac{n_{F}\left(\epsilon_{\boldsymbol{k}^{\prime} \lambda^{\prime}}\right)-n_{F}\left(\epsilon_{\boldsymbol{k}^{\prime}-\boldsymbol{q} \lambda}\right)}{\omega+\epsilon_{\boldsymbol{k}^{\prime} \lambda^{\prime}}-\epsilon_{\boldsymbol{k}^{\prime}-\boldsymbol{q} \lambda}+i 0^{+}}\right\}
\end{aligned}
$$

This is general so far. Next we impose the symmetry requirements of the bands about $\epsilon=0$, i.e. $\epsilon_{\boldsymbol{k}^{\prime}, \lambda}=-\epsilon_{\boldsymbol{k}^{\prime},-\lambda}$ and $\tilde{J}_{\boldsymbol{k}^{\prime}, \lambda}=-\tilde{J}_{\boldsymbol{k}^{\prime},-\lambda}$, and then change the band labels as $r^{\prime}=-\lambda, r=-\lambda^{\prime}$ in Eq. (3.10). Finally, using the relation $n_{F}\left(\epsilon_{\boldsymbol{k}^{\prime},-r}\right)=$ $1-n_{F}\left(\epsilon_{\boldsymbol{k}^{\prime}, r}\right)$ valid for the undoped case $\mu=0$, we arrive at $\Gamma=-\Gamma$, i.e. $\Gamma(q, \omega) \equiv 0$. This result holds true for any type of spectrum where the two bands have a mirror symmetry across $\epsilon=0$, and any bilayer system with one or both of the layers having such a band symmetry with zero doping always results in an overall vanishing drag at all temperatures.

\subsection{Drag in extrinsic graphene systems}

\subsubsection{Analytical results}

We now move on to the drag between finite-doped graphene layers. First we provide a derivation for the exact analytical result of the nonlinear susceptibility Eq. (3.9). In the following we take Fermi energy $\varepsilon_{F}>0$. To faciliate calculation, 
Eq. (3.9) can be written as

$$
\begin{aligned}
\Gamma(\boldsymbol{q}, \omega)= & \tau \sum_{\lambda, \lambda^{\prime}= \pm} \sum_{k}\left\{\left[J_{\lambda \lambda}(\boldsymbol{k}+\boldsymbol{q})-J_{\lambda^{\prime} \lambda^{\prime}}(\boldsymbol{k})\right]\right. \\
& \operatorname{Im}\left\{\left[1+\lambda \lambda^{\prime} \cos \left(\phi_{\boldsymbol{k}+\boldsymbol{q}}-\phi_{\boldsymbol{k}}\right)\right] \frac{n_{F}\left(\xi_{\boldsymbol{k} \lambda^{\prime}}\right)}{\omega+\xi_{\boldsymbol{k} \lambda^{\prime}}-\xi_{\boldsymbol{k}+\boldsymbol{q} \lambda}+i 0^{+}}\right\} \\
& -\left[J_{\lambda \lambda}(\boldsymbol{k})-J_{\lambda^{\prime} \lambda^{\prime}}(\boldsymbol{k}-\boldsymbol{q})\right] \\
& \left.\operatorname{Im}\left\{\left[1+\lambda \lambda^{\prime} \cos \left(\phi_{\boldsymbol{k}}-\phi_{\boldsymbol{k}-\boldsymbol{q}}\right)\right] \frac{n_{F}\left(\xi_{\boldsymbol{k} \lambda}\right)}{\omega+\xi_{\boldsymbol{k}-\boldsymbol{q} \lambda^{\prime}}-\xi_{\boldsymbol{k} \lambda}+i 0^{+}}\right\}\right\} .
\end{aligned}
$$

We now separate Eq. (3.11) into two terms $\Gamma_{\text {intra }}$ and $\Gamma_{\text {inter }}$, corresponding to intraband contribution and interband contribution, respectively:

$$
\Gamma(\boldsymbol{q}, \omega)=\Gamma_{\text {intra }} \theta(v q-\omega)+\Gamma_{\text {inter }} \theta(\omega-v q) .
$$

The intraband contribution, corresponding to terms in Eq. (3.11) with $\lambda=\lambda^{\prime}$, can be written as

$$
\begin{aligned}
\Gamma_{\text {intra }}= & \tau \sum_{k}\left\{\left[J_{++}(\boldsymbol{k}+\boldsymbol{q})-J_{++}(\boldsymbol{k})\right]\right. \\
\times & \operatorname{Im}\left\{\left[1+\cos \left(\phi_{\boldsymbol{k}+\boldsymbol{q}}-\phi_{\boldsymbol{k}}\right)\right] \frac{n_{F}\left(\xi_{\boldsymbol{k}+}\right)}{\omega+\xi_{\boldsymbol{k}+}-\xi_{\boldsymbol{k}+\boldsymbol{q}+}+i 0^{+}}\right\} \\
& -\left[J_{++}(\boldsymbol{k})-J_{++}(\boldsymbol{k}-\boldsymbol{q})\right] \\
\times & \operatorname{Im}\left\{\left[1+\cos \left(\phi_{\boldsymbol{k}}-\phi_{\boldsymbol{k}-\boldsymbol{q}}\right)\right] \frac{n_{F}\left(\xi_{\boldsymbol{k}+}\right)}{\omega+\xi_{\boldsymbol{k}-\boldsymbol{q}+}-\xi_{\boldsymbol{k}+}+i 0^{+}}\right\} \\
& +\left[J_{--}(\boldsymbol{k}+\boldsymbol{q})-J_{--}(\boldsymbol{k})\right] \\
\times & \operatorname{Im}\left\{\left[1+\cos \left(\phi_{\boldsymbol{k}+\boldsymbol{q}}-\phi_{\boldsymbol{k}}\right)\right] \frac{n_{F}\left(\xi_{\boldsymbol{k}-}\right)}{\omega+\xi_{\boldsymbol{k}-}-\xi_{\boldsymbol{k}+\boldsymbol{q}+}+i 0^{+}}\right\} \\
& -\left[J_{--}(\boldsymbol{k})-J_{--}(\boldsymbol{k}-\boldsymbol{q})\right] \\
\times & \left.\operatorname{Im}\left\{\left[1+\cos \left(\phi_{\boldsymbol{k}}-\phi_{\boldsymbol{k}-\boldsymbol{q}}\right)\right] \frac{n_{F}\left(\xi_{\boldsymbol{k}-}\right)}{\omega+\xi_{\boldsymbol{k}-\boldsymbol{q}-}-\xi_{\boldsymbol{k}+}+i 0^{+}}\right\}\right\} .
\end{aligned}
$$

At zero temperature, $n_{F}\left(\xi_{k-}\right)=1$, the last two terms in Eq. (3.13) cancel each other. This is a direct consequence of forbidden intraband transition within the 
valence band since all states are already occupied. In the following, we assume $\omega>0$. The first term (I) intra in Eq. (3.13) can be evaluated as follows:

$$
\begin{aligned}
(\mathrm{I})_{\text {intra }}= & -\frac{\pi \tau}{(2 \pi)^{2}} 2 e v \int_{0}^{\infty} k \mathrm{~d} k \int_{0}^{2 \pi}\left(\frac{q+k \cos \phi_{\boldsymbol{k}}}{|\boldsymbol{k}+\boldsymbol{q}|}-\cos \phi_{\boldsymbol{k}}\right)\left(1+\frac{k+q \cos \phi_{\boldsymbol{k}}}{|\boldsymbol{k}+\boldsymbol{q}|}\right) \\
& \theta\left(\varepsilon_{F}-\epsilon_{\boldsymbol{k}}\right) \delta\left(\omega+\epsilon_{\boldsymbol{k}}-\epsilon_{\boldsymbol{k}+\boldsymbol{q}}\right) \\
= & -\frac{e \tau}{\pi q} \int_{0}^{k_{F}} \mathrm{~d} k \frac{\theta\left(1-\left|t_{1}\right|\right)}{\sqrt{1-t_{1}^{2}}}\left(q-\frac{\omega}{v} t_{1}\right)\left(\frac{\omega / v+2 k+q t_{1}}{\omega / v+k}\right)
\end{aligned}
$$

where in the last line the angular integration has already been carried out and $t_{1} \equiv\left[(\omega / v+k)^{2}-k^{2}-q^{2}\right] / 2 k q$ is the value of $\cos \phi_{\boldsymbol{k}}$ that the delta function in the first line of Eq. (3.14) picks up. Similarly, the second term (II) $)_{\text {intra }}$ can be evaluated:

$$
(\mathrm{II})_{\mathrm{intra}}=-\frac{e \tau}{\pi q} \int_{0}^{k_{F}} \mathrm{~d} k \frac{\theta\left(1-\left|t_{2}\right|\right)}{\sqrt{1-t_{2}^{2}}}\left(q-\frac{\omega}{v} t_{2}\right)\left(\frac{-\omega / v+2 k-q t_{2}}{-\omega / v+k}\right),
$$

where $t_{2} \equiv\left[k^{2}+q^{2}-(-\omega / v+k)^{2}\right] / 2 k q$. Combining Eqs. (3.13)-(3.15), we obtain the intraband term as

$$
\begin{aligned}
\Gamma_{\text {intra }}= & \frac{e \tau}{\pi q}\left[\int_{(q+\omega / v) / 2}^{k_{F}} \mathrm{~d} k \frac{1}{\sqrt{1-t_{2}^{2}}}\left(q-\frac{\omega}{v} t_{2}\right)\left(\frac{2 k-\omega / v-q t_{2}}{k-\omega / v}\right)\right. \\
& \left.-\int_{(q-\omega / v) / 2}^{k_{F}} \mathrm{~d} k \frac{1}{\sqrt{1-t_{1}^{2}}}\left(q-\frac{\omega}{v} t_{1}\right)\left(\frac{2 k+\omega / v+q t_{1}}{k+\omega / v}\right)\right] .
\end{aligned}
$$

For notational clarity, we define the following dimensionless quantities: $x=q / k_{F}$ and $u=\omega / \varepsilon_{F}$, and $\left.\tilde{\Gamma}=\Gamma /\left(2 e k_{F} \tau / \pi\right)\right)$. Eq. (3.16) can then be further simplified as

$$
\begin{aligned}
\tilde{\Gamma}_{\text {intra }}= & \frac{1}{2 \sqrt{x^{2}-u^{2}}} \int_{(x+u) / 2}^{1} \mathrm{~d} k \frac{1}{k-u}\left(x-u \frac{x^{2}-u^{2}+2 k u}{2 k x}\right) \\
& \sqrt{4 k^{2}-4 k u-\left(x^{2}-u^{2}\right)}-\{u \rightarrow-u\} .
\end{aligned}
$$


Carrying out the integration over $k$, we finally obtain the intraband contribution as

$$
\begin{aligned}
\tilde{\Gamma}_{\text {intra }}(x, u)= & \frac{1}{4 x} \sqrt{x^{2}-u^{2}}\{2 \sqrt{(u+x-2)(u-x-2)} \\
& -\sqrt{x^{2}-u^{2}}\left[\tan ^{-1}\left[\frac{\sqrt{(u+x-2)(u-x-2)} \sqrt{x^{2}-u^{2}}}{x^{2}-2-(u-2) u}\right]\right. \\
& \left.\left.+\pi \theta\left[u(u-2)-x^{2}+2\right]\right]\right\} \theta(2-x-u)-\{u \rightarrow-u\} .
\end{aligned}
$$

We now calculate the interband contribution $\Gamma_{\text {inter }}$, which corresponds to terms in Eq. (3.11) with $\lambda=-\lambda^{\prime}$ and can be written as:

$$
\begin{aligned}
\Gamma_{\text {inter }}= & \tau \sum_{k}\left\{\left[J_{++}(\boldsymbol{k}+\boldsymbol{q})-J_{--}(\boldsymbol{k})\right]\right. \\
\times & \operatorname{Im}\left\{\left[1+\cos \left(\phi_{\boldsymbol{k}+\boldsymbol{q}}-\phi_{\boldsymbol{k}}\right)\right] \frac{n_{F}\left(\xi_{\boldsymbol{k}-}\right)}{\omega+\xi_{\boldsymbol{k}-}-\xi_{\boldsymbol{k}+\boldsymbol{q}+}+i 0^{+}}\right\} \\
& -\left[J_{++}(\boldsymbol{k})-J_{--}(\boldsymbol{k}-\boldsymbol{q})\right] \\
\times & \operatorname{Im}\left\{\left[1+\cos \left(\phi_{\boldsymbol{k}}-\phi_{\boldsymbol{k}-\boldsymbol{q}}\right)\right] \frac{n_{F}\left(\xi_{\boldsymbol{k}+}\right)}{\omega+\xi_{\boldsymbol{k}-\boldsymbol{q}-}-\xi_{\boldsymbol{k}+}+i 0^{+}}\right\} \\
& +\left[J_{--}(\boldsymbol{k}+\boldsymbol{q})-J_{++}(\boldsymbol{k})\right] \\
\times & \operatorname{Im}\left\{\left[1+\cos \left(\phi_{\boldsymbol{k}+\boldsymbol{q}}-\phi_{\boldsymbol{k}}\right)\right] \frac{n_{F}\left(\xi_{\boldsymbol{k}+}\right)}{\omega+\xi_{\boldsymbol{k}+}-\xi_{\boldsymbol{k}+\boldsymbol{q}-}+i 0^{+}}\right\} \\
& -\left[J_{--}(\boldsymbol{k})-J_{++}(\boldsymbol{k}-\boldsymbol{q})\right] \\
\times & \left.\operatorname{Im}\left\{\left[1+\cos \left(\phi_{\boldsymbol{k}}-\phi_{\boldsymbol{k}-\boldsymbol{q}}\right)\right] \frac{n_{F}\left(\xi_{\boldsymbol{k}-}\right)}{\omega+\xi_{\boldsymbol{k}-\boldsymbol{q}+}-\xi_{\boldsymbol{k}-}+i 0^{+}}\right\}\right\} .
\end{aligned}
$$

For $\omega>0$, the third and fourth terms in Eq. (3.19) are zero. The first term (I) inter in Eq. (3.19) can be evaluated as follows:

$$
(\mathrm{I})_{\text {inter }}=-\frac{e \tau}{\pi q} \int_{0}^{\infty} \mathrm{d} k \frac{\theta\left(1-\left|t_{2}\right|\right)}{\sqrt{1-t_{2}^{2}}}\left(q-\frac{\omega}{v} t_{2}\right)\left(\frac{\omega / v-2 k+q t_{2}}{\omega / v-k}\right)
$$

and the second term (II) $)_{\text {inter }}$ follows similarly:

$$
(\mathrm{II})_{\text {inter }}=-\frac{e \tau}{\pi q} \int_{0}^{k_{F}} \mathrm{~d} k \frac{\theta\left(1-\left|t_{2}\right|\right)}{\sqrt{1-t_{2}^{2}}}\left(q-\frac{\omega}{v} t_{2}\right)\left(\frac{\omega / v-2 k+q t_{2}}{\omega / v-k}\right) .
$$


Combining Eqs. (3.19)-(3.21), we obtain the interband term as

$$
\begin{aligned}
\Gamma_{\text {inter }}= & -\frac{e \tau}{\pi q}\left[\int_{(\omega / v-q) / 2}^{(\omega / v+q) / 2} \mathrm{~d} k+\int_{(\omega / v-q) / 2}^{\min \left[k_{F},(\omega / v+q) / 2\right]} \mathrm{d} k\right] \\
& \frac{1}{\sqrt{1-t_{2}^{2}}}\left(q-\frac{\omega}{v} t_{2}\right)\left(\frac{\omega / v-2 k+q t_{2}}{w / v-k}\right)
\end{aligned}
$$

which can be simplified further as follows:

$$
\begin{aligned}
\tilde{\Gamma}_{\text {inter }}= & -\frac{1}{2 \sqrt{u^{2}-x^{2}}}\left[\int_{(u-x) / 2}^{(x+u) / 2} \mathrm{~d} k+\int_{(u-x) / 2}^{\min [1,(x+u) / 2]} \mathrm{d} k\right] \\
& \frac{1}{u-k}\left[x-\frac{u}{2 k x}\left(x^{2}-u^{2}+2 u k\right)\right] \sqrt{x^{2}-u^{2}+4 u k-4 k^{2}} .
\end{aligned}
$$

Carrying out the integral over $k$, we finally obtain the following interband contribution

$$
\begin{aligned}
\tilde{\Gamma}_{\text {inter }}(x, u)= & -\frac{1}{4 x} \sqrt{u^{2}-x^{2}}\{2 \sqrt{(x+u-2)(x-u+2)} \\
& +\sqrt{u^{2}-x^{2}}\left[\tan ^{-1}\left[\frac{\sqrt{(x+u-2)(x-u+2)} \sqrt{u^{2}-x^{2}}}{x^{2}-2-(u-2) u}\right]\right. \\
& \left.\left.-\pi \theta\left[x^{2}-2-(u-2) u\right]\right]\right\} \theta(x+u-2) \theta(x-u+2) .
\end{aligned}
$$

The intraband contribution Eq. (3.18) correponds to electron-hole excitations in the vicinity of the Fermi level within the conduction band, which occur at $\omega<v q$; whereas the interband contribution Eq. (3.24) corresponds to electron-hole excitations from the valence band to the conduction band, which occur at $\omega>v q$. Using Eqs. (3.3)-(3.4), (3.12), (3.18), (3.24) and the expression for the graphene polarizability Eq. (2.5), we have calculated numerically the drag resistivity $\rho_{\mathrm{D}}$ for different values of interlayer distance $d$ and density $n$ (Fig. 3.5). Before we proceed to discuss our numerical results, it is instructive to obtain an analytical formula for the drag resistivity under certain approximations. To this end, we first define the standard 
dimensionless parameter for the excitation energy $y=u / x=\omega / v q$ in the Fermi liquid theory, which is the ratio of the phase velocity of the excitation $\omega / q$ to the quasiparticle velocity $v$. At low temperatures and with large interlayer separation, the dominant contribution to the drag conductivity Eq. (3.2) comes from the region with small $q$ and $\omega$; consequently the nonlinear susceptibility can be evaluated in the limits of long wavelength $x \ll 1$ and low energy $y \ll 1$, allowing a closed-form expression for $\Gamma(q, \omega)$ to be extracted. The interband $\left(\lambda=-\lambda^{\prime}\right)$ contribution in Eq. (3.9) is in general smaller than the intraband $\left(\lambda=\lambda^{\prime}\right)$ contribution by $\mathcal{O}\left(x^{2}\right)$, and vanishes in the limit $y \ll 1$, as seen from Eq. (3.12). This is because, in the presence of a finite Fermi level, electrons take more energy to transition from the valence band to the conduction band (interband) than to transition within the conduction band (intraband), and with a small excitation energy the channel of interband transition becomes inaccessible. With the above assumptions, Eq. (3.9) can be evaluated as

$$
\begin{aligned}
\tilde{\Gamma}(x, u=y x) & =\tilde{\Gamma}_{\text {intra }}=-4 y\left[(1-y x) \frac{t_{+} \theta\left(1-\left|t_{+}\right|\right)}{\sqrt{1-t_{+}^{2}}}-(1+y x) \frac{t_{-} \theta\left(1-\left|t_{-}\right|\right)}{\sqrt{1-t_{-}^{2}}}\right] \\
& \simeq-4 y x
\end{aligned}
$$

where $t_{ \pm}=y \pm x\left(1-y^{2}\right) / 2$. Eq. (3.25) is larger than the corresponding expression for the nonlinear susceptibility in regular 2DEG by a factor of 4 , due to an extra $2 \times 2$ degrees of freedom coming from the spin and valley degeneracies in graphene, in addition to the two sublattice degrees of freedom which give rise to the conduction and valence bands. 
The longitudinal conductivity can be obtained from the impurity-dressed current $\boldsymbol{J}=\left(\tau_{\text {tr }} / \tau\right) e v \hat{\boldsymbol{\sigma}}$ using the Kubo formula to give $\sigma_{\mathrm{L}}=e^{2} \nu\left(\varepsilon_{F}\right) D$, where $\nu\left(\varepsilon_{F}\right)=$ $2 k_{F} / \pi v$ is the graphene density of states and $D=v^{2} \tau_{\text {tr }} / 2$ is the diffusion constant. This Kubo formula result is identical with the Boltzmann theory result $\sigma_{\mathrm{L}}=\left(e^{2} / \hbar\right) 2 \varepsilon_{F} \tau_{\text {tr }} / h$. Incidentally, for short-range impurities the transport time is related to the lifetime simply by $\tau_{\mathrm{tr}}=2 \tau$ due to the suppression of backscattering from impurities in graphene. There are two types of disorder in substrate-mounted graphene, one being the charged impurities coming from the substrate; and the other being the neutral impurities intrinsic to the graphene layer itself. In theory, the type of disorder essentially boils down to the expression of the transport time $\tau_{\text {tr }}$ in $\sigma_{\mathrm{L}}$, which yield different types of functional dependence of the conductivity $\sigma_{\mathrm{L}}$ on the carrier density. In experiments, the conductivity is observed to increase linearly with density, a fact alluding to the dominance of the charged impuritity scattering in substrate-mounted graphene samples. We emphasize that this dependence on different types of disorder does not affect the expression of the drag resistivity as the transport time $\tau_{\text {tr }}$ is explicitly canceled out between $\sigma_{\mathrm{D}}$ and $\sigma_{\mathrm{L} 1} \sigma_{\mathrm{L} 2}$. Therefore, our calculation and conclusions apply equally to bilayer systems with substrate-mounted (where charged impurity scattering plays the more dominant role) or suspended graphene samples (where there is only neutral impurity scattering).

In the expression of the drag conductivity Eq. (3.2), the dominant contribution of the integral comes from the region where $q d \lesssim 1$, and for large interlayer separation $d$ satisfying $d^{-1} \ll k_{F}, q_{\mathrm{TF}}$, the interlayer potential Eq. (3.3) can 
be approximated as $U_{12} \simeq q /\left[4 \pi e^{2} \sinh (q d) \Pi_{1}^{c c} \Pi_{2}^{c c}\right]$. Furthermore, the denominator $\sinh ^{2}\left(\omega / 2 k_{B} T\right)$ in Eq. (3.2) also restricts the upper limit of the $\omega$ integral to a few $\sim k_{B} T$; therefore at low temperatures only small values of $\omega / \varepsilon_{F}$ contribute to Eq. (3.2). As a consequence, the polarizability for doped graphene can be approximated by the static screening result $\Pi^{\mathrm{cc}}(q, \omega) \simeq \Pi^{\mathrm{cc}}(q, 0)=\nu\left(\varepsilon_{F}\right)$. Now, using Eq. (3.25) for the nonlinear susceptibility, the drag resistivity is obtained as

$$
\rho_{\mathrm{D}}=\frac{h}{e^{2}} \frac{\pi \zeta(3)}{32} \frac{\left(k_{B} T\right)^{2}}{\varepsilon_{F 1} \varepsilon_{F 2}} \frac{1}{\left(q_{\mathrm{TF} 1} d\right)\left(q_{\mathrm{TF} 2} d\right)} \frac{1}{\left(k_{F 1} d\right)\left(k_{F 2} d\right)}
$$

where $q_{\mathrm{TF}}=4 e^{2} k_{F} / v$ is the Thomas-Fermi wavenumber for extrinsic graphene [28]. The drag resistivity Eq. (3.26), valid for low temperatures $T \ll T_{F}$ and high density and/or large interlayer separation $k_{F} d \gg 1$, has exactly the same form as in the regular 2DEG drag, exhibiting the same dependences of temperature $\left(\sim T^{2}\right)$, interlayer separation $\left(\sim 1 / d^{4}\right)$ and density $\left(\sim\left(n_{1} n_{2}\right)^{-3 / 2}\right)$.

\subsubsection{Numerical results}

Our numerical calculations and analytical results Eq. (3.26) are compared in Fig. 3.4, showing that Eq. (3.26) becomes an increasingly accurate approximation to the full numerical results with increasing values of $k_{F} d$. The fact that the exact numerical results shown in Fig. 3.4 disagree more strongly with the analytic result of Eq. (3.26) for smaller values of $k_{F} d$ is understandable, since the analytic formula given in Eq. (3.26) applies only in the asymptotic $k_{F} d \gg 1$ limit, and for lower carrier density and/or interlayer separation, Eq. (3.26) simply does not apply. In particular, for $k_{F} d=1$, the exact numerical result for Coulomb drag is a factor 
of 4 larger than that given by Eq. (3.26). This trend of an increasing quantitative failure of the asymptotic analytic drag formula for lower values of $k_{F} d$ has also been noted in the literature [50] in the context of low-density hole drag in bilayer p-GaAs 2D systems. For small $k_{F} d$, backscattering effects in Coulomb drag, which are unimportant for $k_{F} d \gg 1$, become important.

On the other hand, our numerical results also show that the temperature dependence of $\rho_{\mathrm{D}}$ remains very close to $T^{2}$ within a wide range of temperatures for typical experimental values of $d$ and $n$ (e.g. $k_{F} d=5$ with $n=5 \times 10^{11} \mathrm{~cm}^{-2}$ and $d \simeq$ $400 \AA$ ). The ratio of the Fermi temperature for graphene to that for regular 2DEG with parabolic spectrum (with effective mass $\mathrm{m}$ ) is $T_{F}$ (graphene) $/ T_{F}(2 \mathrm{DEG})=$ $m v / \hbar \sqrt{\pi n}$, so for low densities e.g. $n=10^{11} \mathrm{~cm}^{-2}, T_{F}=430 \mathrm{~K}$ for graphene can be larger by an order of magnitude than $T_{F}=42 \mathrm{~K}$ for GaAs 2DEG. The temperature dependence of $\rho_{\mathrm{D}}$ for graphene drag therefore remains very closely $T^{2}$ up to about several tens of kelvin where the low temperature regime $T \ll T_{F}$ still remains valid, whereas for drag in regular 2DEG systems departure from the $T^{2}$ dependence of $\rho_{\mathrm{D}}$ typically occurs at $T \lesssim 10 \mathrm{~K}$. The drag resistivity is calculated numerically for various values of $d$ and $n$ and higher values of temperature up to $T=0.2 T_{F}$ (Fig. 3.5); $\rho_{\mathrm{D}}$ is seen to grow slower and slower than $T^{2}$ as temperature increases. Similar dependence on temperature is also observed for drag in regular 2DEG bilayer systems before $T$ reaches $\gtrsim 0.2 T_{F}$, beyond which $\rho_{\mathrm{D}} / T^{2}$ starts to increase due to plasmon enhancement to the drag resistivity $[51,52]$. We discuss the effect of plasmon enhancement to the Coulomb drag in graphene bilayer systems in the following. 


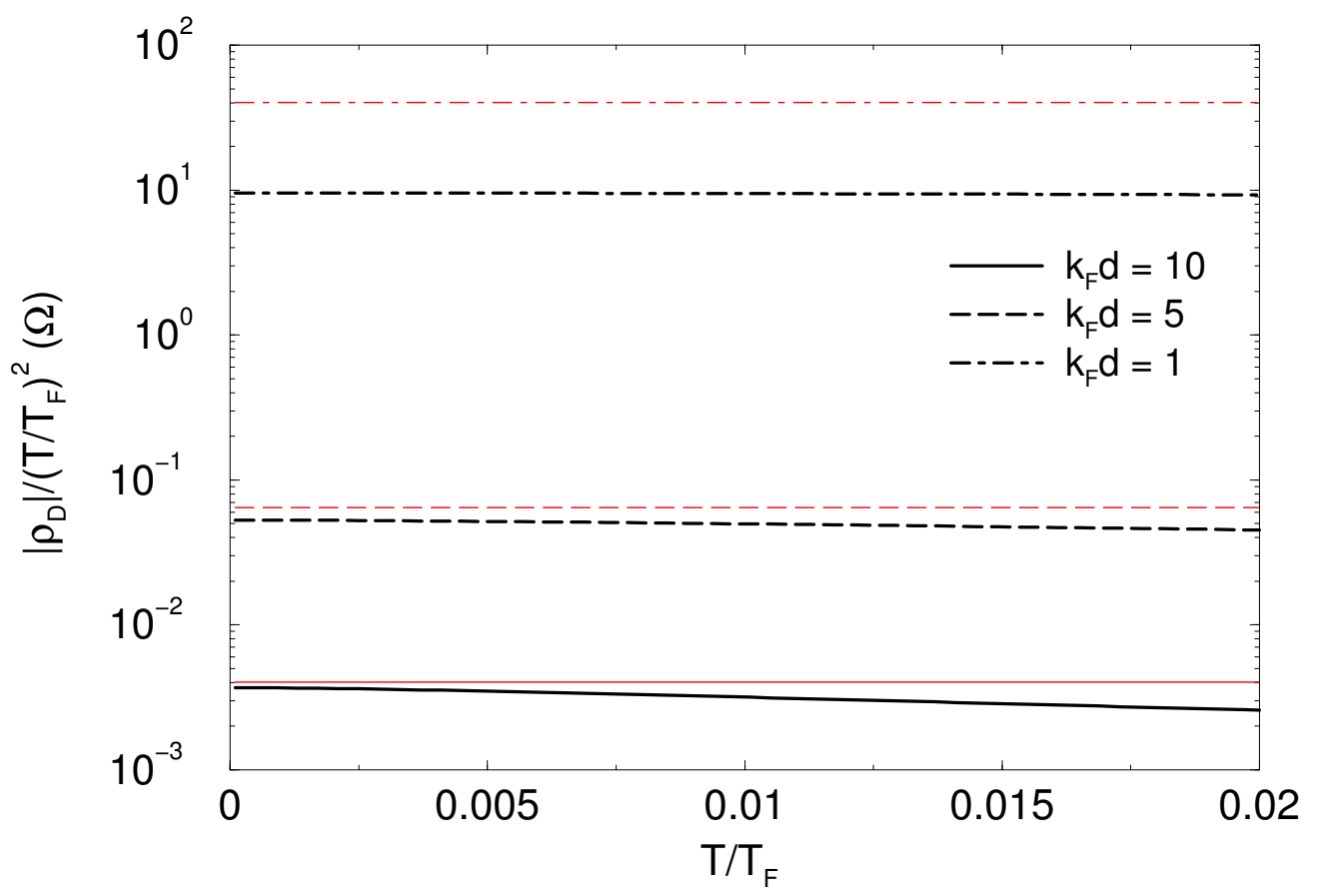

Figure 3.4: $\rho_{\mathrm{D}} /\left(T / T_{F}\right)^{2}$ as a function of $T / T_{F}$ for Coulomb drag between two identical extrinsic graphene sheets, with values of $k_{F} d=10$ (solid lines), 5 (dashed lines), and 1 (dot-dashed lines). Numerical results are indicated with bold (black) lines and analytical results Eq. (3.15) with thin (grey/red) lines. The analytical results become an increasingly accurate approximation to the full numerical results with increasing $k_{F} d$ (i.e. increasing $n$ or $d$ ).

\subsection{Effect of interlayer plasmon excitation}

In regular 2D bilayer systems, enhancement to the drag resistivity due to coupled plasmon modes comes into play with increasing temperature [51, 52]. There exist two plasmon modes, the so-called acoustic and optical modes, for which the electrons on the two layers move collectively in phase and out of phase, respectively, with each other. The energy dispersion lines for these plasmon modes lie above the electron-hole excitation continuum (i.e., the region of $\omega$ vs. $q$ where the imaginary part of the polarizability is non-zero, $\left.\operatorname{Im} \Pi^{\mathrm{cc}}(q, \omega) \neq 0\right)$ at zero temperature, and are not excited at low temperatures. They can be excited, however, at higher 


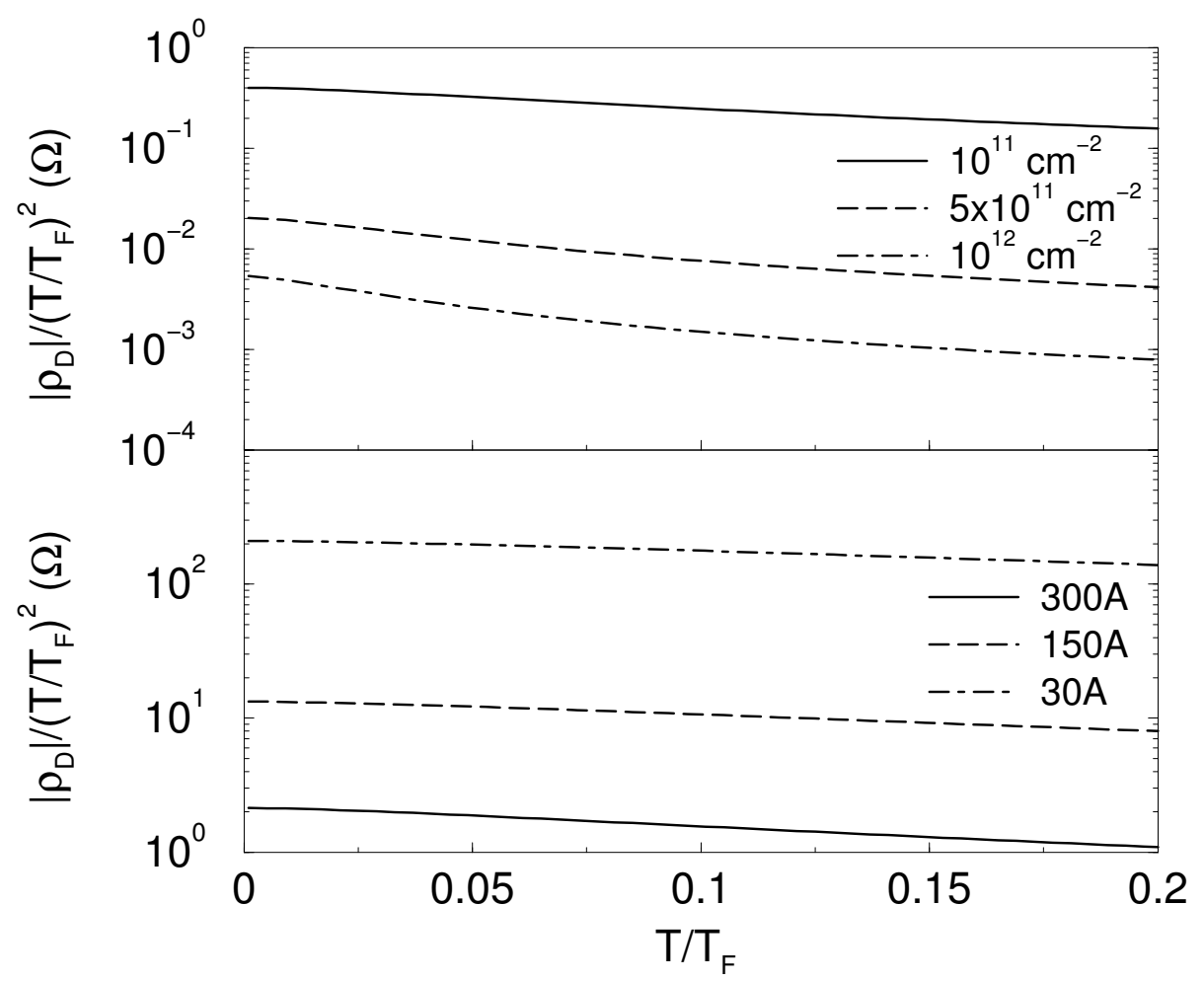

Figure 3.5: $\rho_{\mathrm{D}} /\left(T / T_{F}\right)^{2}$ vs. $T / T_{F}$ for higher values of $T$ up to $0.2 T_{F}$. Upper panel: for fixed interlayer distance $d=500 \AA$ and different values of density $n=10^{11} \mathrm{~cm}^{-2}$ (solid line), $5 \times 10^{11} \mathrm{~cm}^{-2}$ (dashed line), $10^{12} \mathrm{~cm}^{-2}$ (dot-dashed line), corresponding to $T_{F}=431 \mathrm{~K}, 963 \mathrm{~K}, 1361 \mathrm{~K}$ respectively; lower panel: for fixed density $n=10^{11} \mathrm{~cm}^{-2}$ and different values of interlayer distance $d=300 \AA$ (solid line), $150 \AA$ (dashed line) and $30 \AA$ (dot-dashed line).

temperatures when the electron-hole excitation continuum occupies higher values of the excitation energy $\omega$, a consequence of the increasing gradient with increasing momentum $k$ in the parabolic energy dispersion relation. Absorption or emission of a plasmon can occur when the electron-hole excitation continuum starts to overlap with the plasmon dispersion. On the other hand for graphene, because the gradient of the linear dispersion relation is constant, increasing temperature does not increase the range of the possible intraband excitation energies, the electron-hole excitation continuum being always bounded by $\omega<v q$. This means that the plasmon excitation energy will always be out of reach from the intraband excitation channel at all 
temperatures. However, the case is different with the interband excitation, for which the electron-hole excitation continuum overlaps already at $T=0$ with the plasmon dispersion at about $[6] \omega \gtrsim \varepsilon_{F}$. This means that plasmon-induced enhancement of the drag resistivity in graphene occurs, solely due to interband transitions, at a temperature $T \gtrsim T_{F}$; whereas for regular $2 \mathrm{D}$ systems plasmon-induced enhancement occurs already before $T$ reaches $T_{F}$ (at about $\left.T \simeq 0.5 T_{F}\right)$.

Concluding this chapter, we have formulated the Coulomb drag problem for graphene bilayers. The drag resistivity is zero for intrinsic graphene. For extrinsic graphene, the interband contribution to the drag due to electron-hole excitations is suppressed at low temperatures, and the Coulomb drag is due predominantly to the intraband contribution near the Fermi surface in the conduction band. We have obtained exact analytical results at $T=0$ for both intraband and interband contributions to the nonlinear susceptibility, and obtained the drag resistivity numerically. We have also derived an approximate analytical result for the drag resistivity valid for low temperatures, high density and/or large interlayer separation. We find both similarities and differences for the graphene drag resistivity compared with that for regular 2DEG with quadratic energy spectrum. At low temperatures, graphene drag resistivity exhibits the same temperature, bilayer distance and density dependences as regular $2 \mathrm{D}$ systems. For low densities $n \lesssim 10^{11} \mathrm{~cm}^{-2}$, the low temperature regime where the $T^{2}$ dependence of the drag resisitivity holds extends by an order of magnitude that for regular 2D systems, as the Fermi temperature is higher for the same carrier density in graphene than in regular $2 \mathrm{D}$ systems. In contrast to 
regular 2D bilayer systems, there is no contribution to plasmon-induced enhancement of the drag resistivity due to intraband excitations, and the only contribution to plasmon-induced enhancement comes from interband excitations, which occur at temperatures $T \gtrsim \varepsilon_{F}$. The coupled plasmon modes in graphene bilayer systems can therefore be probed experimentally with drag resistivity measurements at high enough temperatures or at low densities. 


\section{Chapter 4}

\section{Quasiparticle renormalization due}

\section{to electron-phonon interaction}

\subsection{Electron-phonon interaction}

As we have seen in Chapter 1, the graphene band structure obtained from the ARPES experiments [4] consists of two kink structures (Fig. 1.7), one at $200 \mathrm{meV}$ away from the Fermi level and the other at the Dirac point. We are interested in the first kink of the band structure in this dissertation, for which electron-phonon interaction has been suggested as the origin in the experimental paper [4, 24]. With this as a motivation, we formulate the self-energy based on the phononmediated electron-electron interaction in graphene, and calculate the interactioninduced renormalization of the band structure, showing that a kink will indeed result due to electron-phonon interaction effect. We shall focus on extrinsic graphene since only extrinsic graphene is realized in experiments. 
It has been suggested in experiments $[4,24]$ that the $200 \mathrm{meV}$ kink in the observed graphene band structure originates from the G band in-plane optical phonon. The G band optical phonon in graphene has long been known in the Raman spectrum for bulk graphite [53], and corresponds to a real space in-plane lattice vibration mode as shown in Fig. 4.1. Fig. 4.2 shows the measured Raman spectrum of graphite and graphene with a peak at a wavenumber of $1580 \mathrm{~cm}^{-1}$ corresponding to the $\mathrm{G}$ band optical phonon. The phonon energy which corresponds to the wavenumber of $1580 \mathrm{~cm}^{-1}$ is $\omega_{0}=200 \mathrm{meV}$.

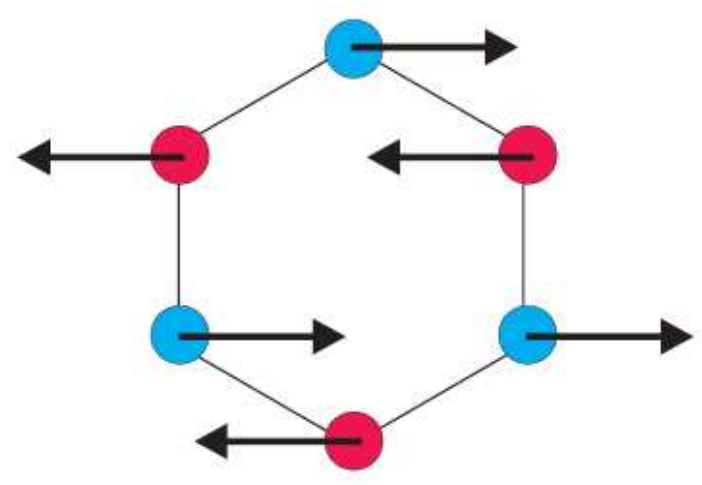

Figure 4.1: The G band in-plane optical phonon mode of graphene. The A-sublattice atoms and B-sublattice atoms vibrate in opposite direction with each other.

In the following, we investigate the effect of electron-phonon interactions on band structure renormalization by calculating the electron self-energy due to the phonon-mediated electron-electron interaction. Since we have considered the effect of Coulomb interaction in Chapter 2, in this chapter we focus on the effect of only electron-phonon interaction on the quasiparticle properties ignoring Coulomb 


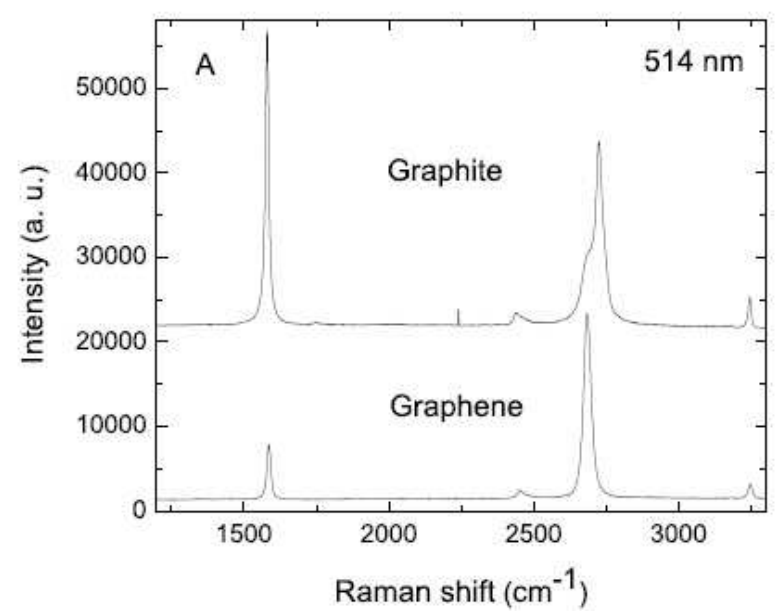

Figure 4.2: Raman spectrum obtained from experiment [5] for graphite and graphene. The peak at the wavenumber $1580 \mathrm{~cm}^{-1}$ is the $\mathrm{G}$ peak corresponding to the zone-center in-plane optical phonons; the band at $2700 \mathrm{~cm}^{-1}$ is the $\mathrm{G}^{\prime}$ band corresponding to a second order Raman scattering of the zone-boundary phonons.

interaction. Our non-interacting system therefore comprises the free electron Hamiltonian and the free phonon Hamiltonian,

$$
\tilde{H}_{0}^{\prime}=\sum_{\boldsymbol{k}} \boldsymbol{c}_{\boldsymbol{k}}^{\dagger} H_{0} \boldsymbol{c}_{\boldsymbol{k}}+\sum_{\boldsymbol{q}} \omega_{0}\left(d_{\boldsymbol{q}}^{\dagger} d_{\boldsymbol{q}}+\frac{1}{2}\right)
$$

where the tilde over $H_{0}^{\prime}$ denotes a quantity expressed in the pseudospin (i.e., sublattice) basis, and $\boldsymbol{c}_{\boldsymbol{k}}=\left[\begin{array}{ll}a_{\boldsymbol{k}} & b_{\boldsymbol{k}}\end{array}\right]^{\mathrm{T}}$ is the two-component electron annihilation operator in the momentum space for the $\mathrm{A}$ and $\mathrm{B}$ sublattices, and $d_{\boldsymbol{q}}, d_{\boldsymbol{q}}^{\dagger}$ are the phonon annihilation and creation operators.

The electron-phonon interaction in graphene in the context of carbon nanotube was studied in Refs. $[54,55,19,20,56]$. The electron-phonon interaction Hamiltonian (in the first quantization) for the $\Gamma$ point optical phonon can be derived using a tight-binding model Refs. $[20,56]$ as

$$
\tilde{v}_{\mathrm{ep}}=g \sum_{\boldsymbol{q}} \boldsymbol{M}(\boldsymbol{q})\left(d_{\boldsymbol{q}}+d_{-\boldsymbol{q}}^{\dagger}\right) e^{i \boldsymbol{q} \cdot \boldsymbol{r}}
$$


where $\boldsymbol{M}(\boldsymbol{q})$ is a matrix in the pseudospin space and is given, for LO/TO phonon, by

$$
\boldsymbol{M}(\boldsymbol{q})=\left[\begin{array}{cc}
0 & M_{\mathrm{AB}} e^{-i \phi_{\boldsymbol{q}}} \\
M_{\mathrm{BA}} e^{i \phi_{\boldsymbol{q}}} & 0
\end{array}\right]
$$

with $M_{\mathrm{AB}}=-1$ or $i$ and $M_{\mathrm{BA}}=1$ or $i$ for $\mathrm{LO}$ or $\mathrm{TO}$ phonons, respectively, and $\phi_{\boldsymbol{q}}=\tan ^{-1}\left(q_{y} / q_{x}\right)$ the azithmuthal angle of the momentum $\boldsymbol{q}$. Optical phonon modes in graphene couple neighboring A-sublattice and B-sublattice carbon atoms through bond stretching and bending, so that the electron-phonon coupling becomes an off-diagonal matrix in the pseudospin space $[19,20] g \boldsymbol{M}$, where the coupling constant $g$ is given by

$$
g=-\left(\frac{\beta \hbar v}{a^{2}}\right) \sqrt{\frac{\hbar}{2 N M_{c} \omega_{0}}},
$$

with $\omega_{0}=200 \mathrm{meV}$ the optical phonon frequency for graphene from Raman scattering experiments $[13,5], N$ the number of unit cells, $M_{c}=2.2 \times 10^{4} m_{e}$ the mass of a carbon atom ( $m_{e}$ is the electron mass), $a=1.42 \AA$ the equilibrium bond length between adjacent carbon atoms and $\beta=\mathrm{d} \ln t / \mathrm{d} \ln l \sim 2$ is a dimensionless parameter that gives the change of the nearest-neighbour hopping energy $t$ with respect to the bond length $l[19,20]$. Similar tight-binding analyses give the form of the electron-phonon interaction Hamiltonian for the $\Gamma$ point acoustic phonon [19], and for the $K$ point phonon modes [57].

We then write the second-quantized form of the electron-phonon interaction 
Eq. (4.2) in the momentum space as:

$$
\begin{aligned}
\tilde{V}_{\mathrm{ep}} & =g \sum_{\boldsymbol{k}, \boldsymbol{q}} \boldsymbol{c}_{\boldsymbol{k}+\boldsymbol{q}}^{\dagger} \boldsymbol{M}(\boldsymbol{q}) \boldsymbol{c}_{\boldsymbol{k}} A_{\boldsymbol{q}} \\
& =g \sum_{\boldsymbol{k}, \boldsymbol{q}} \boldsymbol{c}_{\boldsymbol{k}+\boldsymbol{q}}^{\dagger} \boldsymbol{M}(\boldsymbol{q}) \boldsymbol{c}_{\boldsymbol{k}}\left(d_{\boldsymbol{q}}+d_{-\boldsymbol{q}}^{\dagger}\right)
\end{aligned}
$$

where we have defined $A_{\boldsymbol{q}}=d_{\boldsymbol{q}}+d_{-\boldsymbol{q}}^{\dagger}$ as the phonon displacement operator. We note that Eq. (4.5) has the standard form of the electron-phonon interaction, except that the electron-phonon coupling is given by a matrix which is a function of $\phi_{\boldsymbol{q}}$ instead of simply a constant. Eqs. (4.3)-(4.5) say that the electron-electron scattering via phonon emission/absorption flips the pseudospin (i.e. sublattice) quantum number from $\mathrm{A}$ to $\mathrm{B}$ or vice versa.

\subsection{Phonon-mediated electron-electron interaction}

The electron-phonon interaction $\tilde{V}_{\text {ep }}$ could be transformed to an equivalent phonon-mediated electron-electron interaction $\tilde{V}_{\mathrm{ee}}^{\mathrm{ph}}$ by the following argument. The non-interacting Hamiltonian of the system is given by Eq. (4.1). With the electronphonon interaction Eq. (4.5) turned on, the interacting Green function is given by the standard perturbation series in the interaction picture representation [34]:

$$
\begin{aligned}
& \tilde{G}_{\boldsymbol{k} \lambda}(\tau)=-\left[\sum_{m=0}^{\infty} \frac{(-1)^{m}}{m !} \int_{0}^{\beta} \mathrm{d} \tau_{1} \cdots \int_{0}^{\beta} \mathrm{d} \tau_{m}\left\langle T_{\tau} \tilde{V}_{\text {ep }}\left(\tau_{1}\right) \cdots \tilde{V}_{\text {ep }}\left(\tau_{m}\right) c_{\boldsymbol{k} \lambda}(\tau) c_{\boldsymbol{k} \lambda}^{\dagger}(0)\right\rangle_{0}\right] \\
& /\left[\sum_{m=0}^{\infty} \frac{(-1)^{m}}{m !} \int_{0}^{\beta} \mathrm{d} \tau_{1} \cdots \int_{0}^{\beta} \mathrm{d} \tau_{m}\left\langle T_{\tau} \tilde{V}_{\text {ep }}\left(\tau_{1}\right) \cdots \tilde{V}_{\text {ep }}\left(\tau_{m}\right)\right\rangle_{0}\right]
\end{aligned}
$$

where $\beta=1 / k_{\mathrm{B}} T, T_{\tau}$ is the imaginary time ordering operator, and the brackets $\langle\cdots\rangle$ indicates thermal average with respect to $\exp \left(-\beta \tilde{H}_{0}^{\prime}\right)$. The thermal average of 
the integrand in the $m^{\text {th }}$ term of, say, the denominator in Eq. (4.6) decouples into a product of a phononic and an electronic thermal average,

$$
\begin{aligned}
& \left\langle T_{\tau} A_{\boldsymbol{q}_{1}}\left(\tau_{1}\right) \cdots A_{\boldsymbol{q}_{m}}\left(\tau_{m}\right) c_{\boldsymbol{k}+\boldsymbol{q}_{1} \lambda_{1}}^{\dagger}\left(\tau_{1}\right) c_{\boldsymbol{k} \lambda_{1}^{\prime}}\left(\tau_{1}\right) \cdots c_{\boldsymbol{k}+\boldsymbol{q}_{m} \lambda_{m}}^{\dagger}\left(\tau_{m}\right) c_{\boldsymbol{k} \lambda_{m}^{\prime}}\left(\tau_{m}\right)\right\rangle_{0} \\
= & \left\langle T_{\tau} A_{\boldsymbol{q}_{1}}\left(\tau_{1}\right) \cdots A_{\boldsymbol{q}_{m}}\left(\tau_{m}\right)\right\rangle_{0}\left\langle T_{\tau} c_{\boldsymbol{k}+\boldsymbol{q}_{1} \lambda_{1}}^{\dagger}\left(\tau_{1}\right) c_{\boldsymbol{k} \lambda_{1}^{\prime}}\left(\tau_{1}\right) \cdots c_{\boldsymbol{k}+\boldsymbol{q}_{m} \lambda_{m}}^{\dagger}\left(\tau_{m}\right) c_{\boldsymbol{k} \lambda_{m}^{\prime}}\left(\tau_{m}\right)\right\rangle_{0} .
\end{aligned}
$$

Noticing that only an even number of phonon displacement operators will lead to a non-zero contribution in Eq. (4.6), and by Wick's theorem, the thermal average of the product of phonon displacement operators factorizes into a product of pairs of displacement operators

$$
\begin{aligned}
& g^{2} M_{\alpha^{\prime} \alpha}\left(\boldsymbol{q}_{i}\right) M_{\beta^{\prime} \beta}\left(\boldsymbol{q}_{j}\right)\left\langle T_{\tau} A_{\boldsymbol{q}_{i}}\left(\tau_{i}\right) A_{\boldsymbol{q}_{j}}\left(\tau_{j}\right)\right\rangle_{0} \\
= & g^{2} M_{\alpha^{\prime} \alpha}\left(\boldsymbol{q}_{i}\right) M_{\beta^{\prime} \beta}\left(\boldsymbol{q}_{j}\right)\left\langle T_{\tau} A_{\boldsymbol{q}_{i}}\left(\tau_{i}\right) A_{-\boldsymbol{q}_{i}}\left(\tau_{j}\right)\right\rangle_{0} \delta_{\boldsymbol{q}_{j},-\boldsymbol{q}_{i}} \\
= & g^{2} M_{\alpha^{\prime} \alpha}\left(\boldsymbol{q}_{i}\right) M_{\beta^{\prime} \beta}\left(-\boldsymbol{q}_{i}\right) \mathcal{D}_{\boldsymbol{q}}^{0}\left(\tau_{i}-\tau_{j}\right) \delta_{\boldsymbol{q}_{j},-\boldsymbol{q}_{i}},
\end{aligned}
$$

where we have introduced the non-interacting phonon Green function $\mathcal{D}^{0}\left(q, \tau_{1}-\tau_{2}\right) \equiv$ $-\left\langle T_{\tau} A_{\boldsymbol{q}_{1}}\left(\tau_{1}\right) A_{\boldsymbol{q}_{2}}^{\dagger}\left(\tau_{2}\right)\right\rangle_{0}=-\left\langle T_{\tau} A_{\boldsymbol{q}_{1}}\left(\tau_{1}\right) A_{-\boldsymbol{q}_{2}}\left(\tau_{2}\right)\right\rangle_{0}$. The interacting Green function Eq. (4.6) becomes

$$
\begin{aligned}
& \tilde{G}_{\boldsymbol{k} \lambda}(\tau)=-\left[\sum_{n=0}^{\infty} \frac{(-1)^{n}}{n !} \int_{0}^{\beta} \mathrm{d} \tau_{1} \cdots \int_{0}^{\beta} \mathrm{d} \tau_{n}\left\langle T_{\tau} \tilde{V}_{\mathrm{ee}}^{\mathrm{ph}}\left(\tau_{1}\right) \cdots \tilde{V}_{\mathrm{ee}}^{\mathrm{ph}}\left(\tau_{n}\right) c_{\boldsymbol{k} \lambda}(\tau) c_{\boldsymbol{k} \lambda}^{\dagger}(0)\right\rangle_{0}\right] \\
& /\left[\sum_{n=0}^{\infty} \frac{(-1)^{n}}{n !} \int_{0}^{\beta} \mathrm{d} \tau_{1} \cdots \int_{0}^{\beta} \mathrm{d} \tau_{n}\left\langle T_{\tau} \tilde{V}_{\mathrm{ee}}^{\mathrm{ph}}\left(\tau_{1}\right) \cdots \tilde{V}_{\mathrm{ee}}^{\mathrm{ph}}\left(\tau_{n}\right)\right\rangle_{0}\right]
\end{aligned}
$$

where $n=m / 2$ because of the phonon displacement operator pairing Eq. (4.8), and

$$
\tilde{V}_{\mathrm{ee}}^{\mathrm{ph}}=\frac{1}{2} g^{2} \sum_{k_{1}, k_{2}, q} \mathcal{D}^{0}\left(q, \tau_{2}-\tau_{1}\right) M_{\alpha^{\prime} \alpha}(\boldsymbol{q}) M_{\beta^{\prime} \beta}(-\boldsymbol{q}) c_{\boldsymbol{k}_{\mathbf{1}}+\boldsymbol{q} \alpha^{\prime}}^{\dagger} c_{\boldsymbol{k}_{\mathbf{2}}-\boldsymbol{q} \beta^{\prime}}^{\dagger} c_{k_{1} \alpha} c_{k_{2} \beta},
$$


is the phonon-mediated electron-electron interaction operator we are looking for (summation over repeated indices is implied). We note that Eq. (4.10) can also be derived by starting from the Hamiltonian $\tilde{H}_{0}^{\prime}+\tilde{V}_{\mathrm{ph}}$ within the functional integral approach and integrating out the phonon degrees of freedom. Eq. (4.10) is schematically shown in the diagrammatic language in Fig. 4.3, from which the meaning of the equation is clear: two electrons scatter off one another through the exchange of a phonon, and by virtue of the off-diagonal electron-phonon interaction vertex $g \boldsymbol{M}$, the pseudospin of each electron is flipped after the scattering. Eq. (4.10) is the

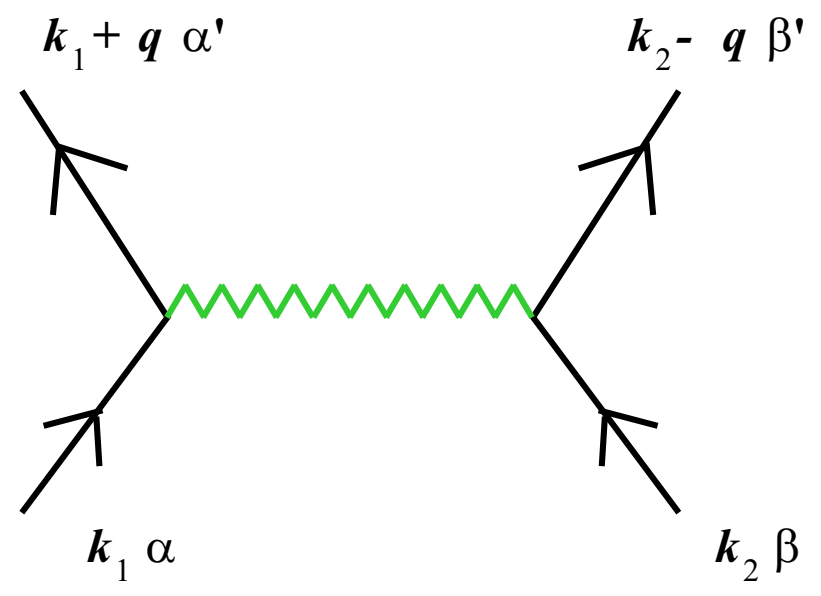

Figure 4.3: Feynman diagram for the phonon-mediated electron-electron interaction Eq. (4.10). The solid straight lines denote the electron Green function, and the zigzag line denotes the phonon Green function. The electron-phonon interaction vertex is given by $g M_{\alpha^{\prime} \alpha}, g M_{\beta^{\prime} \beta}$ on the two vertices. An electron initially in the state $\left|\boldsymbol{k}_{1} \alpha\right\rangle$ interacts with another electron in the state $\left|\boldsymbol{k}_{2} \beta\right\rangle$ through exchanging a phonon of momentum $\boldsymbol{q}$, and then the first electron is scattered off to the state $\left|\boldsymbol{k}_{1}+\boldsymbol{q} \alpha^{\prime}\right\rangle$ while the second electron is scattered off to the state $\left|\boldsymbol{k}_{2}-\boldsymbol{q} \beta^{\prime}\right\rangle$. Since the electron-phonon interaction vertices $g M_{\alpha^{\prime} \alpha}, g M_{\beta^{\prime} \beta}$ are off-diagonal matrices, the pseudospin labels of the two electrons are flipped in the process: $\alpha^{\prime}=-\alpha$ and $\beta^{\prime}=-\beta$.

central quantity of this chapter from which other quantities such as self-energy are derived. To find the self-energy for electrons in the conduction band/holes in the valence band, we work in the chiral basis (i.e. the basis where the Hamiltonian $H_{0}$ is 
diagonal). We shall focus ourselves only on LO phonons, as the calculations for TO phonons parallel that for LO phonons. The matrix elements for electron scattering from chirality $\lambda \rightarrow \lambda^{\prime}$ and $\mu \rightarrow \mu^{\prime}$ through phonon emission/absorption are

$$
\begin{aligned}
\left\langle\boldsymbol{k}+\boldsymbol{q} \lambda^{\prime}|M(\boldsymbol{q})| \boldsymbol{k} \lambda\right\rangle & =\frac{1}{2}\left[\lambda^{\prime} e^{-i\left(\phi_{\boldsymbol{k}+\boldsymbol{q}}-\phi_{\boldsymbol{q}}\right)}-\lambda e^{i\left(\phi_{\boldsymbol{k}}-\phi_{\boldsymbol{q}}\right)}\right], \\
\left\langle\boldsymbol{k}-\boldsymbol{q} \mu^{\prime}|M(-\boldsymbol{q})| \boldsymbol{k} \mu\right\rangle & =\frac{1}{2}\left[-\mu^{\prime} e^{-i\left(\phi_{\boldsymbol{k}-\boldsymbol{q}}-\phi_{\boldsymbol{q}}\right)}+\mu e^{i\left(\phi_{\boldsymbol{k}}-\phi_{\boldsymbol{q}}\right)}\right],
\end{aligned}
$$

and the corresponding phonon-mediated electron-electron interaction in the chiral basis can be written as

$$
\begin{aligned}
& V_{\mathrm{ee}}^{\mathrm{ph}}=\frac{1}{2} g^{2} \sum_{k_{1}, k_{2}, q} \mathcal{D}^{0}\left(q, \tau_{1}-\tau_{2}\right)\left\langle\boldsymbol{k}_{1}+\boldsymbol{q} \lambda^{\prime}|M(\boldsymbol{q})| \boldsymbol{k}_{1} \lambda\right\rangle \\
& \left\langle\boldsymbol{k}_{2}-\boldsymbol{q} \mu^{\prime}|M(-\boldsymbol{q})| \boldsymbol{k}_{2} \mu\right\rangle c_{\boldsymbol{k}_{\mathbf{1}}+\boldsymbol{q} \boldsymbol{\lambda}^{\prime}}^{\dagger} c_{\boldsymbol{k}_{\mathbf{2}}-\boldsymbol{q} \mu^{\prime}}^{\dagger} c_{k_{1} \lambda} c_{k_{2} \mu},
\end{aligned}
$$

where here the operators $c_{k \lambda}^{\dagger}, c_{k \lambda}$ in Eq. (4.12) denotes the electron creation and annihilation operators in the chiral basis, with $\lambda, \mu= \pm 1$ the chirality.

\subsection{Self-energy}

The effective many-body velocity or mass renormalization to the band structure comes in large part from the electron-phonon interaction with the Coulomb interaction yielding a quantitatively small correction [34, 58]. Since the effective velocity renormalization due to screened Coulomb interaction was considered in Chapter 2, we shall focus in this chapter on the many-body effects of the electron-phonon interaction without the effects of Coulomb interaction. The electron self-energy in the chiral basis due to phonon-mediated electron-electron interaction can be derived 
using Eqs. (4.11)-(4.12) as

$$
\begin{aligned}
{\left[\Sigma_{\boldsymbol{k}}\left(i k_{n}\right)\right]_{\mu^{\prime} \lambda}=} & -k_{\mathrm{B}} T \frac{g^{2}}{4} \sum_{q, i q_{n}} \mathcal{D}^{0}\left(i q_{n}\right)\left[\lambda^{\prime} e^{-i\left(\phi_{\boldsymbol{k}+\boldsymbol{q}}-\phi_{\boldsymbol{q}}\right)}-\lambda e^{i\left(\phi_{\boldsymbol{k}}-\phi_{\boldsymbol{q}}\right)}\right] \\
& {\left[-\mu^{\prime} e^{-i\left(\phi_{\boldsymbol{k}}-\phi_{\boldsymbol{q}}\right)}+\mu e^{i\left(\phi_{\boldsymbol{k}+\boldsymbol{q}}-\phi_{\boldsymbol{q}}\right)}\right] G_{\boldsymbol{k}+\boldsymbol{q} \mu}^{0}\left(i k_{n}+i q_{n}\right) \delta_{\mu \lambda^{\prime}}, }
\end{aligned}
$$

where $G_{k \lambda}^{0}\left(i k_{n}\right)=1 /\left(i k_{n}-\xi_{k \lambda}\right)$ is the non-interacting quasiparticle Green function in the chiral basis, with $\xi_{k \lambda}=\lambda \epsilon_{k}-\varepsilon_{F}$ the quasiparticle energy rendered from the Fermi level, and $\mathcal{D}^{0}\left(q, i q_{n}\right)=2 \omega_{0} /\left[\left(i q_{n}\right)^{2}-\omega_{0}^{2}\right]$ is the non-interacting phonon Green function in the frequency domain. From Eq. (4.13), we find that the diagonal elements of the self-energy matrix $\Sigma_{\boldsymbol{k} \pm \pm} \equiv \Sigma_{\boldsymbol{k} \pm}$ are given by

$$
\begin{aligned}
\Sigma_{\boldsymbol{k} \pm}\left(i k_{n}\right)= & -k_{\mathrm{B}} T \frac{g^{2}}{2} \sum_{\lambda} \sum_{q, i q_{n}} \mathcal{D}^{0}\left(i q_{n}\right) G_{\boldsymbol{k}+\boldsymbol{q} \lambda}^{0}\left(i k_{n}+i q_{n}\right) \\
& {\left[1 \mp \lambda \cos \left(\phi_{\boldsymbol{k}+\boldsymbol{q}}-2 \phi_{\boldsymbol{q}}\right)\right], }
\end{aligned}
$$

and the off-diagonal elements of the self-energy matrix

$$
\Sigma_{\boldsymbol{k} \pm \mp}\left(i k_{n}\right)= \pm i k_{\mathrm{B}} T g^{2} \sum_{\lambda} \sum_{q, i q_{n}} G_{\boldsymbol{k}+\boldsymbol{q} \lambda}^{0}\left(i k_{n}+i q_{n}\right) \mathcal{D}^{0}\left(i q_{n}\right) \frac{\lambda \sin \left(\phi_{\boldsymbol{k}+\boldsymbol{q}}-2 \phi_{\boldsymbol{q}}\right)}{2}=0
$$

vanish after performing the angular integration [the same is also true for the case with only Coulomb interaction, c.f. Eq. (2.18)]. For TO phonons, Eq. (4.14) remains the same except the chirality factor becomes $1 \mp \cos \left(\phi_{\boldsymbol{k}+\boldsymbol{q}}-2 \phi_{\boldsymbol{q}}\right) \rightarrow 1 \pm \cos \left(\phi_{\boldsymbol{k}+\boldsymbol{q}}-\right.$ $\left.2 \phi_{\boldsymbol{q}}\right)$. The Matsubara sum in Eq. (4.14) can be performed explicitly:

$$
\begin{aligned}
-k_{\mathrm{B}} T \sum_{i q_{n}} \mathcal{D}^{0}\left(i q_{n}\right) G_{\boldsymbol{k}+\boldsymbol{q} \lambda}^{0}\left(i k_{n}+i q_{n}\right)= & \frac{n_{B}\left(\omega_{0}\right)+n_{F}\left(\xi_{\boldsymbol{k}+\boldsymbol{q} \lambda}\right)}{i k_{n}-\xi_{\boldsymbol{k}+\boldsymbol{q} \lambda}+\omega_{0}} \\
& +\frac{n_{B}\left(\omega_{0}\right)+1-n_{F}\left(\xi_{\boldsymbol{k}+\boldsymbol{q} \lambda}\right)}{i k_{n}-\xi_{\boldsymbol{k}+\boldsymbol{q} \lambda}-\omega_{0}},
\end{aligned}
$$


and we note that at zero temperature, $n_{B}\left(\omega_{0}\right)=0$.

For concreteness, we consider the case of n-doped graphene, $\varepsilon_{F}>0$, and evaluate the self-energy $\Sigma_{\boldsymbol{k}+}$ for electrons in the conduction band. For p-doped material, one should consider $\Sigma_{\boldsymbol{k}-}$ for holes in the valence band.

\subsubsection{Imaginary part of the self-energy}

The quasiparticle decay rate is proportional to the imaginary part of the selfenergy,

$$
\begin{aligned}
\operatorname{Im} \tilde{\Sigma}_{k+}(\omega)= & -\frac{\pi g^{2}}{2} \sum_{k^{\prime}, \lambda}\left\{n_{F}\left(\xi_{k^{\prime} \lambda}\right) \delta\left(\omega-\xi_{k^{\prime} \lambda}+\omega_{0}\right)+\left[1-n_{F}\left(\xi_{k^{\prime} \lambda}\right)\right] \delta\left(\omega-\xi_{k^{\prime} \lambda}-\omega_{0}\right)\right\} \\
& {\left[1-\lambda \cos \left(\phi_{\boldsymbol{k}^{\prime}}-2 \phi_{\boldsymbol{k}-\boldsymbol{k}^{\prime}}\right)\right] }
\end{aligned}
$$

where $\boldsymbol{k}^{\prime}=\boldsymbol{k}+\boldsymbol{q}$. Recalling the trigonometric identities $\cos \left(\phi_{\boldsymbol{k}^{\prime}}-2 \phi_{\boldsymbol{k}-\boldsymbol{k}^{\prime}}\right)=$

$\cos \phi_{\boldsymbol{k}^{\prime}} \cos 2 \phi_{\boldsymbol{k}-\boldsymbol{k}^{\prime}}+\sin \phi_{\boldsymbol{k}^{\prime}} \sin 2 \phi_{\boldsymbol{k}-\boldsymbol{k}^{\prime}}, \cos 2 \phi_{\boldsymbol{k}-\boldsymbol{k}^{\prime}}=1-2 \sin ^{2} \phi_{\boldsymbol{k}-\boldsymbol{k}^{\prime}}$ and $\sin 2 \phi_{\boldsymbol{k}-\boldsymbol{k}^{\prime}}=$ $2 \sin \phi_{\boldsymbol{k}-\boldsymbol{k}^{\prime}} \cos \phi_{\boldsymbol{k}-\boldsymbol{k}^{\prime}}$, and noting that

$$
\begin{aligned}
& k+q \cos \phi_{\boldsymbol{k}-\boldsymbol{k}^{\prime}}=k^{\prime} \cos \phi_{\boldsymbol{k}^{\prime}} \\
& q \sin \phi_{\boldsymbol{k}-\boldsymbol{k}^{\prime}}=k^{\prime} \sin \phi_{\boldsymbol{k}^{\prime}},
\end{aligned}
$$

we obtain the following relation

$$
1-\lambda \cos \left(\phi_{\boldsymbol{k}^{\prime}}-2 \phi_{\boldsymbol{k}-\boldsymbol{k}^{\prime}}\right)=\left(k+\lambda k^{\prime}\right)^{2} \frac{1-\lambda \cos \phi_{\boldsymbol{k}^{\prime}}}{k^{2}+k^{2}-2 k k^{\prime} \cos \phi_{\boldsymbol{k}^{\prime}}} .
$$

The angular integration in Eq. (4.17) can then be carried out, with

$$
\begin{aligned}
\int_{0}^{2 \pi} \mathrm{d} \phi_{\boldsymbol{k}^{\prime}} \frac{1-\lambda \cos \phi_{\boldsymbol{k}^{\prime}}}{k^{2}+k^{2}-2 k k^{\prime} \cos \phi_{\boldsymbol{k}^{\prime}}} & =\lambda \frac{\pi}{k k^{\prime}}\left(1-\left|\frac{k-\lambda k^{\prime}}{k+\lambda k^{\prime}}\right|\right) \quad\left(k \neq k^{\prime}\right), \\
& =\lambda \frac{\pi}{k^{2}} \quad\left(k=k^{\prime}\right)
\end{aligned}
$$




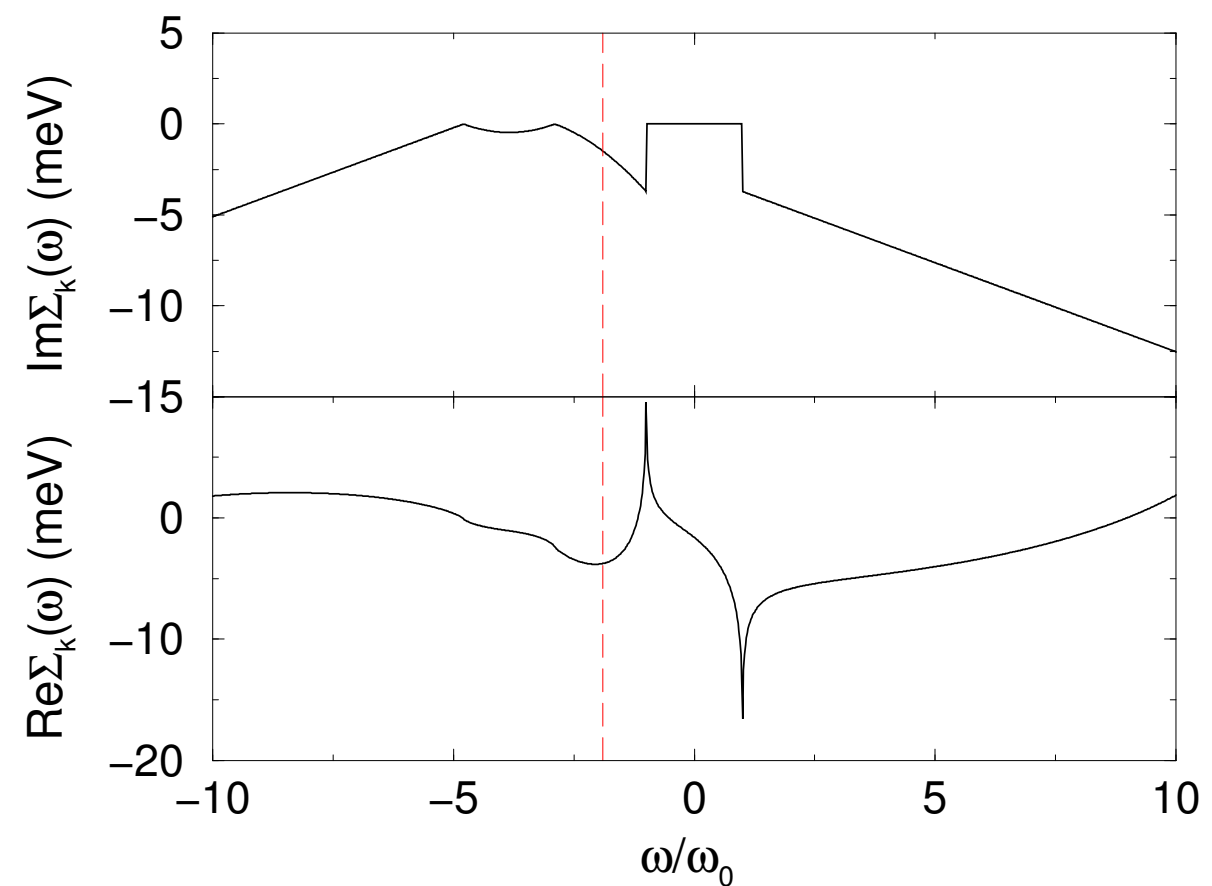

Figure 4.4: The real and imaginary parts of the self-energy at $k=k_{F}$ for density $n=10^{13} \mathrm{~cm}^{-2}$. The red dashed line shows the position of the Dirac point. Region $\omega>\omega_{0}$ corresponds to the intraband term $F_{\text {intra }}\left(k, \omega, \omega_{0}\right)$ whereas $-\omega_{0}-\varepsilon_{F}<\omega<$ $-\omega_{0}$ corresponds to the intraband term $F_{\text {intra }}\left(k, \omega,-\omega_{0}\right)$. The first dip in $|\operatorname{Im} \Sigma|$ on the left side of the Dirac point marks the onset of the interband transitions $F_{\text {inter }}$ for $\omega<-\omega_{0}-\varepsilon_{F}$; the second dip occurring at $-\omega_{0}-\epsilon_{k}-\varepsilon_{F}$ originates from the particular form of the angular factor in Eq. (4.14) which comes from the electronphonon interaction vertex Eq. (4.11).

The remaining integration over $k^{\prime}$ can be done easily, and Eq. (4.17) then yields

$$
\begin{aligned}
\operatorname{Im} \Sigma_{\boldsymbol{k}+}(\omega)= & -\frac{1}{8} g_{\mathrm{ee}}^{2}\left[F_{\text {intra }}\left(k, \omega, \omega_{0}\right) \theta\left(\omega-\omega_{0}\right) \theta\left(\omega-\omega_{0}+\varepsilon_{F}\right)\right. \\
& +F_{\text {intra }}\left(k, \omega,-\omega_{0}\right) \theta\left(-\omega-\omega_{0}\right) \theta\left(\omega+\omega_{0}+\varepsilon_{F}\right) \\
& \left.+F_{\text {inter }}\left(k, \omega,-\omega_{0}\right) \theta\left(-\omega-\omega_{0}\right) \theta\left(-\omega-\omega_{0}-\varepsilon_{F}\right)\right],
\end{aligned}
$$

where

$$
g_{\mathrm{ee}}^{2}=\frac{g^{2} \mathcal{A}}{\hbar^{2} v^{2}}=2 \times 10^{-2},
$$


is the dimensionless phonon-mediated electron-electron coupling constant $(\mathcal{A}$ is the area of the sample) [Eq. (1.17)], the term

$F_{\text {intra }}\left(k, \omega, \omega_{0}\right)=\left(\omega-\omega_{0}+\varepsilon_{F}+\epsilon_{k}\right)\left(\omega-\omega_{0}+\varepsilon_{F}+\epsilon_{k}-\left|\omega-\omega_{0}+\varepsilon_{F}-\epsilon_{k}\right|\right) / \epsilon_{k}$,

corresponds to the contribution from quasiparticles making an intraband transition in the vicinity of the Fermi level by emitting a phonon, $F_{\text {intra }}\left(k, \omega,-\omega_{0}\right)$ corresponds to quasiholes making an intraband transition in the vicinity of the Fermi level by absorbing a phonon, and the term

$$
\begin{aligned}
F_{\text {inter }}\left(k, \omega,-\omega_{0}\right)= & -\left|\omega+\omega_{0}+\varepsilon_{F}+\epsilon_{k}\right|\left(\left|\omega+\omega_{0}+\varepsilon_{F}+\epsilon_{k}\right|\right. \\
& \left.+\omega+\omega_{0}+\varepsilon_{F}-\epsilon_{k}\right) / \epsilon_{k},
\end{aligned}
$$

corresponds to quasiparticles making an interband transition from the conduction to the valence band by emitting a phonon.

\subsubsection{Real part of the self-energy}

From Eq. (4.14), We now evaluate the real part of the self-energy, which is given by

$\operatorname{Re} \Sigma_{\boldsymbol{k} \pm}(\omega)=\frac{g^{2}}{2} \sum_{k^{\prime} \lambda}\left[\frac{n_{F}\left(\xi_{\boldsymbol{k}+\boldsymbol{q} \lambda}\right)}{\omega-\xi_{\boldsymbol{k}+\boldsymbol{q} \lambda}+\omega_{0}}+\frac{1-n_{F}\left(\xi_{\boldsymbol{k}+\boldsymbol{q} \lambda}\right)}{\omega-\xi_{\boldsymbol{k}+\boldsymbol{q} \lambda}-\omega_{0}}\right]\left[1 \mp \lambda \cos \left(\phi_{\boldsymbol{k}+\boldsymbol{q}}-2 \phi_{\boldsymbol{q}}\right)\right]$

The sum over $\lambda= \pm 1$ in Eq. (4.25) takes care of the the intraband $\lambda=1$ and interband $\lambda=-1$ contributions. Direct calculation of Eq. (4.25) gives a logarithmically 
divergent result, which is also the case for the calculation of the real part of the selfenergy with only screened Coulomb interaction. This is because in the two-band linear dispersion model for graphene $H_{0}=v \boldsymbol{\sigma} \cdot \boldsymbol{k}$, there exists an infinite number of electrons in the valence band (i.e. the "Dirac sea"), which is obviously unphysical. In reality, the linear band structure of graphene in the vicinity of the K point only holds up to a cut-off energy scale given by the inverse lattice spacing, beyond which the energy dispersion becomes nonlinear. The real part of the self-energy should therefore be regularized by introducing the cut-off energy $\Lambda_{c}=\hbar v k_{c}=\hbar v / a$ in the momentum integral in Eq. (4.25). Using Eq. (4.20) for the angular integral, and then carrying out the $k^{\prime}$ integral, we evaluate the intraband contribution of the first term in Eq. (4.25) as follows:

$$
\begin{aligned}
& (\mathrm{I})_{\text {intra }} \equiv \sum_{k^{\prime}} \frac{n_{F}\left(\xi_{\boldsymbol{k}^{\prime}+}\right)}{\omega-\xi_{\boldsymbol{k}^{\prime}+}+\omega_{0}}\left[1-\cos \left(\phi_{\boldsymbol{k}^{\prime}}-2 \phi_{\boldsymbol{k}^{\prime}-\boldsymbol{k}}\right)\right] \\
= & \frac{1}{(2 \pi)^{2}} \int_{0}^{k_{F}} \mathrm{~d} k^{\prime} k^{\prime} \frac{1}{\omega-\xi_{\boldsymbol{k}^{\prime}+}+\omega_{0}} \frac{\pi}{k k^{\prime}}\left(k+k^{\prime}\right)^{2}\left(1-\left|\frac{k-k^{\prime}}{k+k^{\prime}}\right|\right) \\
= & \frac{1}{4 \pi k}\left\{\int_{0}^{k_{F}} \mathrm{~d} k^{\prime} \frac{1}{\omega-v k^{\prime}+\varepsilon_{F}+\omega_{0}}\left(k+k^{\prime}\right) 2 k^{\prime} \theta\left(k-k_{F}\right)\right. \\
& +\left[\int_{0}^{k} \mathrm{~d} k^{\prime} \frac{1}{\omega-v k^{\prime}+\varepsilon_{F}+\omega_{0}}\left(k+k^{\prime}\right) 2 k^{\prime}\right. \\
& \left.\left.+\int_{k}^{k_{F}} \mathrm{~d} k^{\prime} \frac{1}{\omega-v k^{\prime}+\varepsilon_{F}+\omega_{0}}\left(k+k^{\prime}\right) 2 k\right] \theta\left(k_{F}-k\right)\right\},
\end{aligned}
$$

where we have converted to dimensionless units The first integral which is multiplied by $\theta\left(k-k_{F}\right)$ yields

$$
\begin{aligned}
& \frac{1}{4 \pi k} \int_{0}^{k_{F}} \mathrm{~d} k^{\prime} \frac{1}{\omega-v k^{\prime}+\varepsilon_{F}+\omega_{0}}\left(k+k^{\prime}\right) 2 k^{\prime} \theta\left(k-k_{F}\right) \\
= & \frac{1}{2 \pi \epsilon_{k}} \theta\left(k-k_{F}\right)\left[-\frac{1}{2} \varepsilon_{F}\left(2 \omega+2 \omega_{0}+2 \epsilon_{k}+3 \varepsilon_{F}\right)\right. \\
& \left.+\left(\omega+\omega_{0}+\varepsilon_{F}\right)\left(\omega+\omega_{0}+\epsilon_{k}+\varepsilon_{F}\right) \ln \left(\frac{\omega+\omega_{0}+\varepsilon_{F}}{\omega+\omega_{0}}\right)\right],
\end{aligned}
$$


while the bracketed expression containing the second and third integrals which are together multiplied by $\theta\left(k_{F}-k\right)$ yields

$$
\begin{aligned}
& \frac{1}{4 \pi k}\left[\int_{0}^{k} \mathrm{~d} k^{\prime} \frac{1}{\omega-v k^{\prime}+\varepsilon_{F}+\omega_{0}}\left(k+k^{\prime}\right) 2 k^{\prime}\right. \\
& \left.+\int_{k}^{k_{F}} \mathrm{~d} k^{\prime} \frac{1}{\omega-v k^{\prime}+\varepsilon_{F}+\omega_{0}}\left(k+k^{\prime}\right) 2 k\right] \theta\left(k_{F}-k\right) \\
= & \frac{1}{2 \pi \epsilon_{k}} \theta\left(k_{F}-k\right)\left\{-\frac{1}{2} \epsilon_{k}\left(2 \omega+2 \omega_{0}+4 \varepsilon_{F}+\epsilon_{k}\right)\right. \\
& +\left(\omega+\omega_{0}+\varepsilon_{F}\right)\left(\omega+\omega_{0}+\epsilon_{k}+\varepsilon_{F}\right) \ln \left(\frac{\omega+\omega_{0}+\varepsilon_{F}}{\omega+\omega_{0}-\epsilon_{k}+\varepsilon_{F}}\right) \\
& \left.+\left(\omega+\omega_{0}+\epsilon_{k}+\varepsilon_{F}\right) \epsilon_{k} \ln \left(\frac{\omega+\omega_{0}-\epsilon_{k}+\varepsilon_{F}}{\omega+\omega_{0}}\right)\right\} .
\end{aligned}
$$

The intraband contribution of the second term in Eq. (4.25) gives

$$
\begin{aligned}
& (\mathrm{II})_{\text {intra }} \equiv \sum_{k^{\prime}} \frac{1-n_{F}\left(\xi_{\boldsymbol{k}^{\prime}+}\right)}{\omega-\xi_{\boldsymbol{k}^{\prime}+}-\omega_{0}}\left[1-\cos \left(\phi_{\boldsymbol{k}^{\prime}}-2 \phi_{\boldsymbol{k}^{\prime}-\boldsymbol{k}}\right)\right] \\
= & \frac{1}{(2 \pi)^{2}} \int_{0}^{\infty} \mathrm{d} k^{\prime} k^{\prime} \frac{1-\theta\left(k_{F}-k^{\prime}\right)}{\omega-\xi_{\boldsymbol{k}^{\prime}+}-\omega_{0}} \frac{\pi}{k k^{\prime}}\left(k+k^{\prime}\right)^{2}\left(1-\left|\frac{k-k^{\prime}}{k+k^{\prime}}\right|\right) \\
= & \frac{1}{4 \pi k} \int_{0}^{k} \mathrm{~d} k^{\prime} \frac{2 k^{\prime}}{\omega-\omega_{0}+\varepsilon_{F}-v k^{\prime}}\left(k+k^{\prime}\right)+\frac{1}{2 \pi} \int_{k}^{\infty} \mathrm{d} k^{\prime} \frac{1}{\omega-\omega_{0}+\varepsilon_{F}-v k^{\prime}}\left(k+k^{\prime}\right) \\
& -\left.(\mathrm{I})_{\text {intra }}\right|_{\omega \rightarrow-\omega_{0}} .
\end{aligned}
$$

The first integral gives

$$
\begin{aligned}
& \frac{1}{4 \pi k} \int_{0}^{k} \mathrm{~d} k^{\prime} \frac{2 k^{\prime}}{\omega-\omega_{0}+\varepsilon_{F}-v k^{\prime}}\left(k+k^{\prime}\right) \\
= & \frac{1}{4 \pi \epsilon_{k}}\left[-\epsilon_{k}\left(2 \omega-2 \omega_{0}+2 \varepsilon_{F}+3 \epsilon_{k}\right)\right. \\
& \left.+2\left(\omega-\omega_{0}+\varepsilon_{F}\right)\left(\omega-\omega_{0}+\epsilon_{k}+\varepsilon_{F}\right) \ln \left(\frac{\omega-\omega_{0}+\varepsilon_{F}}{\omega-\omega_{0}+\varepsilon_{F}-\epsilon_{k}}\right)\right],
\end{aligned}
$$

while the second integral gives

$$
\begin{aligned}
& \frac{1}{2 \pi} \int_{k}^{\infty} \mathrm{d} k^{\prime} \frac{1}{\omega-\omega_{0}+\varepsilon_{F}-v k^{\prime}}\left(k+k^{\prime}\right) \\
= & \frac{1}{2 \pi}\left[\epsilon_{k}-\Lambda_{c}+\left(\omega-\omega_{0}+\varepsilon_{F}+\epsilon_{k}\right) \ln \left(\frac{\omega-\omega_{0}+\varepsilon_{F}-\epsilon_{k}}{\omega-\omega_{0}+\varepsilon_{F}-\Lambda_{c}}\right)\right] .
\end{aligned}
$$


Now we calculate the interband contribution of the first term in Eq. (4.25) as follows:

$$
\begin{aligned}
(\mathrm{I})_{\text {inter }} & =\sum_{k^{\prime}} \frac{n_{F}\left(\xi_{\boldsymbol{k}^{\prime}-}\right)}{\omega-\xi_{\boldsymbol{k}^{\prime}-}+\omega_{0}}\left[1+\cos \left(\phi_{\boldsymbol{k}^{\prime}}-2 \phi_{\boldsymbol{k}-\boldsymbol{k}^{\prime}}\right)\right] \\
& =-\frac{1}{(2 \pi)^{2}} \int_{0}^{\infty} \mathrm{d} k^{\prime} k^{\prime} \frac{1}{\omega+v k^{\prime}+\omega_{0}+\varepsilon_{F}} \frac{\pi}{k k^{\prime}}\left|k-k^{\prime}\right|^{2}\left(1-\frac{k+k^{\prime}}{\left|k-k^{\prime}\right|}\right) \\
& =\frac{1}{2 \pi k}\left[\int_{0}^{k} \mathrm{~d} k^{\prime} \frac{k^{\prime}\left(k-k^{\prime}\right)}{\omega+\omega_{0}+\varepsilon_{F}+v k^{\prime}}+k \int_{0}^{\infty} \mathrm{d} k^{\prime} \frac{k^{\prime}-k}{\omega+\omega_{0}+\varepsilon_{F}+v k^{\prime}}\right] .
\end{aligned}
$$

The first integral gives

$$
\begin{aligned}
& \frac{1}{2 \pi k} \int_{0}^{k} \mathrm{~d} k^{\prime} \frac{k^{\prime}\left(k-k^{\prime}\right)}{\omega+\omega_{0}+\varepsilon_{F}+v k^{\prime}} \\
= & \frac{1}{4 \pi \epsilon_{k}}\left[\epsilon_{k}\left(2 \omega+2 \omega_{0}+2 \varepsilon_{F}+\epsilon_{k}\right)\right. \\
& \left.+2\left(\omega+\omega_{0}+\varepsilon_{F}\right)\left(\omega+\omega_{0}+\varepsilon_{F}+\epsilon_{k}\right) \ln \left(\frac{\omega+\omega_{0}+\varepsilon_{F}}{\omega+\omega_{0}+\varepsilon_{F}+\epsilon_{k}}\right)\right],
\end{aligned}
$$

whereas the second integral gives

$$
\begin{aligned}
& \frac{1}{2 \pi} \int_{0}^{\infty} \mathrm{d} k^{\prime} \frac{k^{\prime}-k}{\omega+\omega_{0}+\varepsilon_{F}+v k^{\prime}}=\frac{1}{2 \pi}\left[-\epsilon_{k}+\Lambda_{c}\right. \\
& \left.+\left(\omega+\omega_{0}+\varepsilon_{F}+\epsilon_{k}\right) \ln \left(\frac{\omega+\omega_{0}+\varepsilon_{F}+\epsilon_{k}}{\omega+\omega_{0}+\varepsilon_{F}+\Lambda_{c}}\right)\right] .
\end{aligned}
$$

The interband contribution of the second term in Eq. (4.25) (II) $)_{\text {inter }}=0$ at zero temperature. Combining the results from Eqs. (4.26)-(4.34), we obtain the following 
exact analytical expression for $\operatorname{Re} \Sigma_{\boldsymbol{k}+}(\omega)$ :

$$
\begin{aligned}
& \operatorname{Re} \Sigma_{\boldsymbol{k}+}(\omega)=\frac{1}{4 \pi} g_{\mathrm{ee}}^{2} \frac{1}{\epsilon_{k}}\left\{\left\{\theta ( k - k _ { F } ) \left[-\omega_{0} \varepsilon_{F}\right.\right.\right. \\
& \left.+\left(\omega+\omega_{0}+\varepsilon_{F}\right)\left(\omega+\omega_{0}+\varepsilon_{F}+\epsilon_{k}\right) \ln \left(\frac{\omega+\omega_{0}+\varepsilon_{F}}{\omega+\omega_{0}}\right)\right] \\
& +\theta\left(k_{F}-k\right)\left[-\omega_{0} \epsilon_{k}+\left(\omega+\omega_{0}+\varepsilon_{F}\right)\left(\omega+\omega_{0}+\epsilon_{k}+\varepsilon_{F}\right) \ln \left(\frac{\omega+\omega_{0}+\varepsilon_{F}}{\omega+\omega_{0}+\varepsilon_{F}-\epsilon_{k}}\right)\right. \\
& \left.\left.+\left(\omega+\omega_{0}+\varepsilon_{F}+\epsilon_{k}\right) \epsilon_{k} \ln \left(\frac{\omega+\omega_{0}+\varepsilon_{F}-\epsilon_{k}}{\omega+\omega_{0}}\right)\right]-\left(\omega_{0} \rightarrow-\omega_{0}\right)\right\} \\
& +\epsilon_{k}\left(2 \omega_{0}-\epsilon_{k}\right)+\left(\omega-\omega_{0}+\varepsilon_{F}\right)\left(\omega-\omega_{0}+\varepsilon_{F}+\epsilon_{k}\right) \ln \left(\frac{\omega-\omega_{0}+\varepsilon_{F}}{\omega-\omega_{0}+\varepsilon_{F}-\epsilon_{k}}\right) \\
& +\left(\omega+\omega_{0}+\varepsilon_{F}\right)\left(\omega+\omega_{0}+\varepsilon_{F}+\epsilon_{k}\right) \ln \left(\frac{\omega+\omega_{0}+\varepsilon_{F}}{\omega+\omega_{0}+\varepsilon_{F}+\epsilon_{k}}\right) \\
& +\left(\omega-\omega_{0}+\varepsilon_{F}+\epsilon_{k}\right) \epsilon_{k} \ln \left(\frac{\omega-\omega_{0}+\varepsilon_{F}-\epsilon_{k}}{\omega-\omega_{0}+\varepsilon_{F}-\Lambda_{c}}\right) \\
& \left.+\left(\omega+\omega_{0}+\varepsilon_{F}+\epsilon_{k}\right) \epsilon_{k} \ln \left(\frac{\omega+\omega_{0}+\varepsilon_{F}+\epsilon_{k}}{\omega+\omega_{0}+\varepsilon_{F}+\Lambda_{c}}\right)\right\} .
\end{aligned}
$$

In Fig. 4.4 we show the real and the imaginary parts of the self-energy. The gap in $\operatorname{Im} \Sigma_{\boldsymbol{k}+}(\omega)$ between the phonon energies $-\omega_{0}$ and $\omega_{0}$, characteristic of the optical phonon emission/absorption process, results from the Pauli blocking by electrons located within $\omega_{0}$ of the Fermi level, so that decay by an electron with energy $\omega \in\left[-\omega_{0}, \omega_{0}\right]$ is forbidden because there is no available final state to decay to. Beyond the gap, $\operatorname{Im} \Sigma_{\boldsymbol{k}+}(\omega)$ behaves linearly as $\omega$ for large $\omega / \omega_{0}$ on both sides of the gap due to the linear dependence on energy of the graphene density of states. At $\omega<-\omega_{0}-\varepsilon_{F}$, phonon emission can occur through interband transitions, serving as an extra decay channel in graphene. The interband contribution $F_{\text {inter }}$ has two dips in $\left|\operatorname{Im} \Sigma_{\boldsymbol{k}+}(\omega)\right|$ below the Dirac point (Fig. 4.4), one at $-\omega_{0}-\varepsilon_{F}$ which comes from phase-space restrictions marking the onset of the interband transition and the other at $-\omega_{0}-\epsilon_{k}-\varepsilon_{F}$ which originates from the angle-dependent electron-phonon 
interaction vertex Eq. (4.11). Taking account of both LO and TO contributions to the self-energy, the $\cos \left(\phi_{\boldsymbol{k}+\boldsymbol{q}}-2 \phi_{\boldsymbol{q}}\right)$ term will be canceled out and as a result the second dip in $\left|\operatorname{Im} \Sigma_{\boldsymbol{k}+}(\omega)\right|$ will disappear. In regular semiconductors/metals with a parabolic energy dispersion, the imaginary part of the self-energy is often calculated under the approximation of a constant density of states resulting in a square wellshaped profile $[59,34,58]$, which suffices to model the quasiparticle decay rate quite accurately for these materials. For graphene, however, the linear $\omega$-dependence of $\operatorname{Im} \Sigma_{\boldsymbol{k}+}(\omega)$ away from the gap is a peculiar feature and we believe that a correct modeling of the existing experimental data for the graphene quasiparticle decay rate [4] should take this feature into account.

\subsubsection{Quasiparticle spectral function}

In Fig. 4.5, we show the calculated spectral function $A_{\boldsymbol{k}}(\omega) \equiv-2 \operatorname{Im} G_{\boldsymbol{k}+}(\omega)$, where $G_{\boldsymbol{k}+}(\omega)=1 /\left[\omega-\xi_{k+}-\tilde{\Sigma}_{\boldsymbol{k}+}(\omega)\right]$ is the interacting electron Green function with self-energy correction [c.f. Eq. (2.51)]. The spectral function comprises two contributions: a delta function peak within the gap, and a background beyond the gap. As the value of $k$ increases (decreases), the delta function approaches $\omega_{0}\left(-\omega_{0}\right)$ asymptotically from below (above), whereas the background peak at $\omega>0(\omega<0)$ becomes increasingly prominent. 


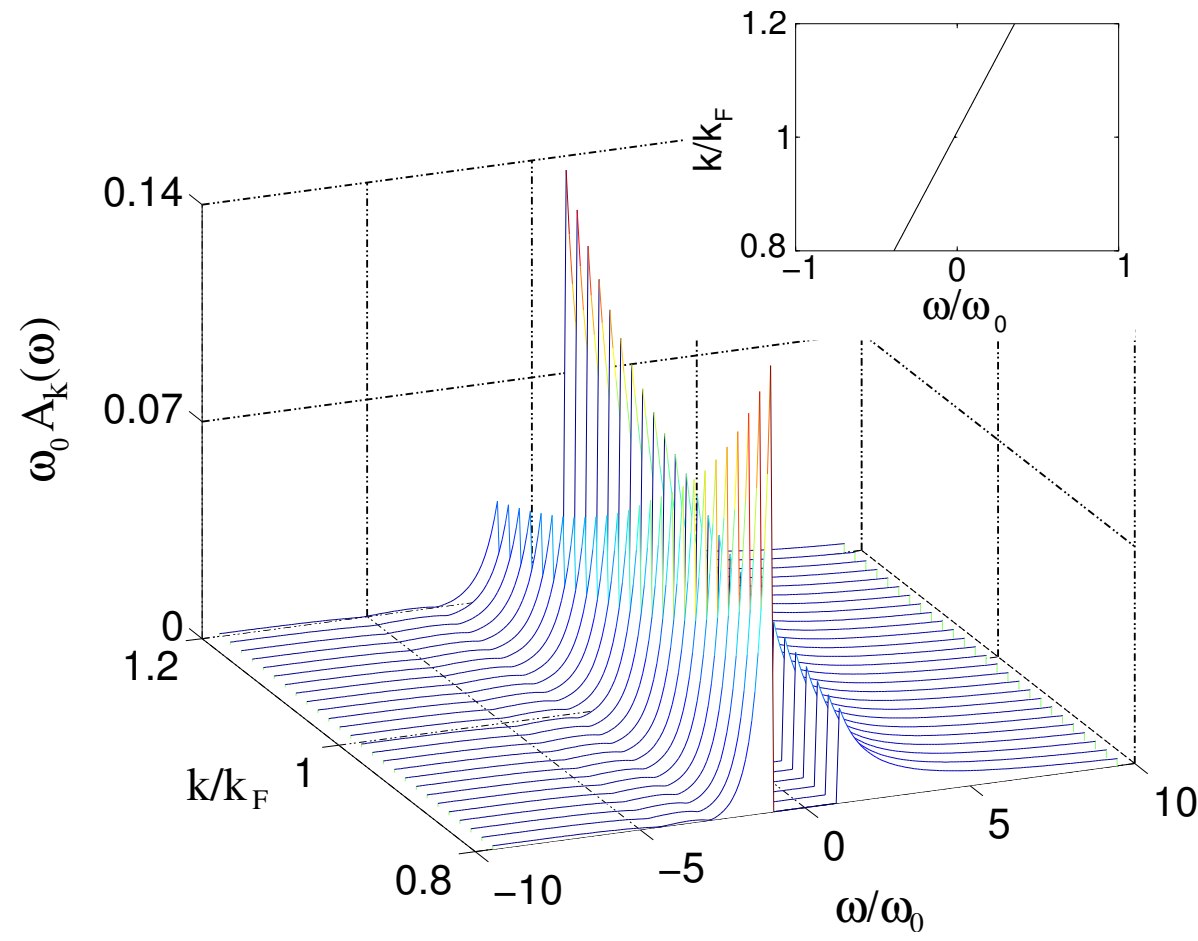

Figure 4.5: The spectral function $A_{k}(\omega)$ (normalized to dimensionless unit by multiplying $\left.\omega_{0}\right)$ as a function of $\omega / \omega_{0}$ and $k / k_{F}$ for density $n=10^{13} \mathrm{~cm}^{-2}$, the inset shows the locus of the position of the quasiparticle delta function inside the gap $\omega / \omega_{0} \in[-1,1]$.

\subsubsection{Renormalized energy dispersion}

The derivative of the real part of the self-energy with respect to $\omega$ and $k$ determines the effective velocity $v^{*}$, which is given by

$$
\frac{v^{*}(k)}{v}=\frac{1+B}{1-A}
$$

where $A=\partial \operatorname{Re} \Sigma_{\boldsymbol{k}}(\omega) /\left.\partial \omega\right|_{\omega=v\left(k-k_{F}\right)}$ and $B=(1 / v) \partial \operatorname{Re} \Sigma_{\boldsymbol{k}}(\omega) /\left.\partial k\right|_{\omega=v\left(k-k_{F}\right)}$. In regular metals with a parabolic band dispersion, the relevant phonons are acoustic phonons with a characteristic energy scale given by the Debye energy $\omega_{\mathrm{D}}$. In calculating $v^{*}$, it is usual practice to ignore $B$ since in metals $\varepsilon_{F} / \omega_{D} \sim 10^{2}$ and $B \sim \Sigma / \varepsilon_{F} \ll A \sim \Sigma / \omega_{D}$. In graphene, however, $\varepsilon_{F} / \omega_{0} \sim 1$ and $B$ should not be neglected in calculating $v^{*} / v$. Although the phonon-mediated electron-electron 
coupling (whose magnitude is given by $g_{\mathrm{ee}}^{2} \sim 10^{-2}$ ) is in general weaker than the Coulomb coupling (given by the interaction parameter [28] $r_{s} \sim 0.7$ ), electronphonon interaction actually contributes more significantly to the effective velocity renormalization than Coulomb interaction, because the real part of the self-energy due to electron-phonon interaction exhibit sharp changes near the phonon energies which are not present in the case of Coulomb interaction. This results in a larger value of the energy derivative of $\operatorname{Re} \Sigma$ near the phonon energy and therefore larger value of $v^{*} / v$. In Eq. (4.35), logarithmic singularities occur in $\operatorname{Re} \Sigma_{\boldsymbol{k}+}(\omega)$ at $\pm \omega_{0}$

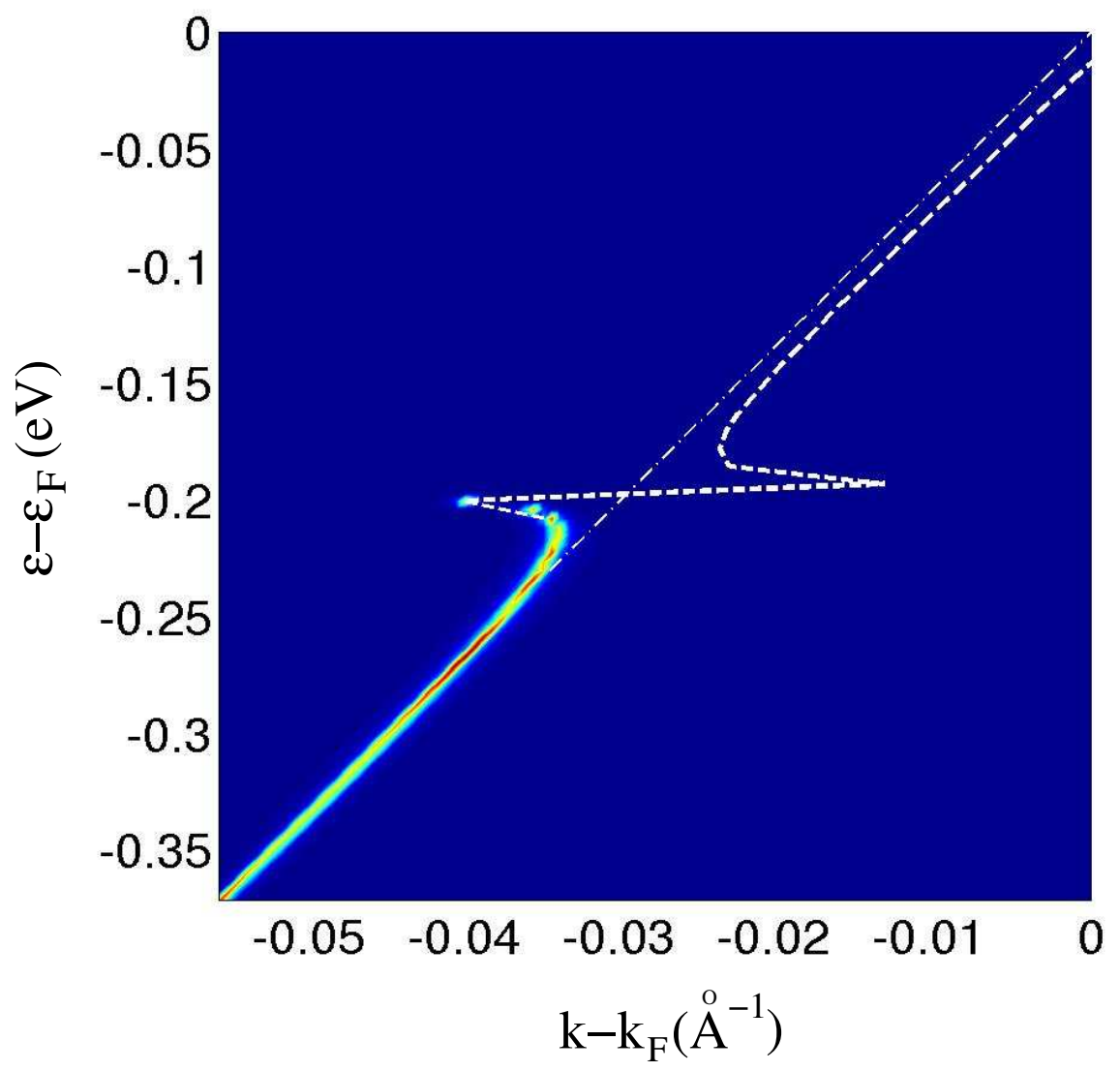

Figure 4.6: The renormalized conduction band energy spectrum for $n=10^{13} \mathrm{~cm}^{-2}$. The colored intensity plot shows the magnitude of the spectral function $A_{k}(\omega)$ whereever it is non-zero, while the dashed white line shows the region within the amount of phonon energy $\omega_{0}=0.196 \mathrm{eV}$ from the Fermi level where $A_{k}(\omega)=0$. The thin dot-dashed straight line shows the bare unrenormalized spectrum. 


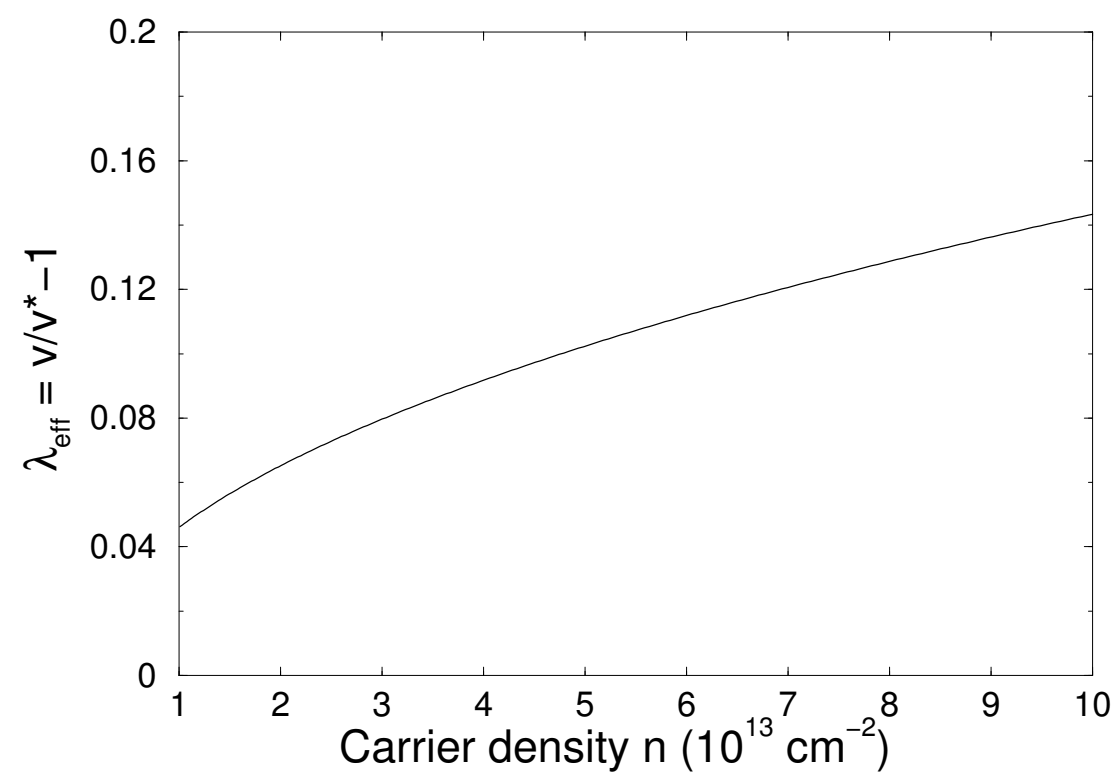

Figure 4.7: The effective electron-phonon "coupling" parameter $\lambda_{\text {eff }}=v / v^{*}\left(k_{F}\right)-1$ as a function of doping $n$.

where $\operatorname{Im} \Sigma_{\boldsymbol{k}+}(\omega)$ goes through a finite step jump, yielding also logarithmic singularities in the derivatives of $\operatorname{Re} \Sigma_{\boldsymbol{k}+}(\omega)$ with respect to $\omega$ and $k$. Fig. 4.6 shows the calculated renormalized energy spectrum for electron densities $n=10^{13} \mathrm{~cm}^{-2}$, the sharp kink shows the logarithmic singularity at $\omega=\omega_{0}$. Such a kink only occurs in the conduction band if the phonon energy is within the Fermi sea $\omega_{0}<\varepsilon_{F}$; if the phonon energy lies outside of the Fermi sea $\omega_{0}>\varepsilon_{F}$ the conduction band will be smooth and the logarithmic singularity could occur in the valence band. Although our theory predicts the correct position of the kink (Fig. 4.6) in agreement with the experiment $[4,24]$, the observed energy spectrum differs from ours in that the sharpness of the kink in the experiment is greatly reduced. First, we note that our zero-temperature theory works well in the temperature regime of the experiment $T<30 \mathrm{~K}$ which is much smaller than the Fermi temperature $T_{F} \sim 4300 \mathrm{~K}$ at $n \sim 10^{13} \mathrm{~cm}^{-2}$, hence smearing of the kink due to finite temperature effect should 
be minor. We believe that the kink in the experimental spectrum is suppressed due to the combined effect of disorder and screening - in particular, disorder effects are considerable in the currently existing graphene samples. The combination of these effects will remove the logarithmic singularity leading to a much smoother kink in the energy spectrum, bringing the calculated spectrum in closer agreement with the observed spectrum. Fig. 4.7 shows the effective electron-phonon "coupling" parameter $[34,58] \lambda_{\text {eff }} \equiv v / v^{*}\left(k_{F}\right)-1$ as a function of electron density $n$. Our results agree in order-of-magnitude with the extracted value of $\lambda_{\text {eff }}$ from the experiment (Fig. 3c in the second reference of Ref. [24]), and the band velocity is shown to be reduced by a percentage of $\left(v-v^{*}\right) / v \sim 4-13 \%$ from $n=10^{13}-10^{14} \mathrm{~cm}^{-2}$.

Concluding this chapter, we have developed a theory for the phonon-mediated electron-electron interaction and the interaction-induced quasiparticle renormalization in graphene. We calculated the electron self-energy, the spectral function and the band velocity renormalization due to the electron-phonon interaction. Our results show that the electron-phonon coupling has a large effect on the band structure renormalization, exhibiting a kink at $\sim 200 \mathrm{meV}$ below the Fermi surface as observed in the experiment and a reduction of the band velocity by $\sim 4 \%-13 \%$ at the experimental doping level. 


\section{Chapter 5}

\section{Phonon renormalization due to electron-phonon interaction}

The many-body renormalization of the phonon spectrum due to the electronphonon interaction is an important open question. Experimentally, Raman scattering offers a means to measure the long-wavelength phonon energy whereas X-ray and neutron scattering provide an avenue to probe the entire phonon energy dispersion. Due to free carrier-induced many-body interactions, one expects the observed phonon energy in a doped system to be different from the "bare" phonon energy in an undoped system. Physically, electrons respond to the dynamical lattice vibrations by screening the lattice potential, changing the elastic constants of these vibrational modes and thereby renormalizing the phonon energy. Traditionally, the Bohn-Oppenheimer (BO) approximation, also going by the name of static or adiabatic approximation, has been an important assumption in the calculation of the phonon energy dispersion, which requires the phonon energy to be much smaller than the characteristic energy scale for the electrons, usually the Fermi energy. Recently, 
a number of Raman scattering experiments $[18,60,61,62]$ on extrinsic graphene (where the electron density can be tuned by gating) have emerged, with the observed density dependence of the Raman shift (for the long-wavelength G-band optical phonons at the $\Gamma$ point) pointing to the inapplicability of the $\mathrm{BO}$ approximation in graphene. This density dependence has been addressed for the long-wavelength phonons (i.e. $q=0$ ) using perturbation theory $[18,62]$ and density-functional theory [63].

An interesting question remains as to whether the Kohn anomaly (KA) [64], which appears as a cusp in the phonon energy dispersion at $q=2 k_{F}$ for ordinary 2D metals, will be substantially modified in graphene due to its quasi-relativistic chiral band structure. The occurrence of KA is entirely a many-body effect as it originates from the screening of the electron-phonon interaction by electrons, and correpsonds to the singularities of the phonon self-energy or its derivatives as a function of $q$. For graphite, the phonon energy dispersion has been studied with a number of experimental technqiues (X-ray scattering, neutron scattering, double resonance Raman scattering and electron loss energy spectroscopy) in a number of papers $[65,66,67,68,69,70,71,72]$. Theoretically, Ref. [73] calculated the graphite phonon energy dispersion taking account of many-body renormalization using density-functional theory. Ref. [63] addresses the phonon energy renormalization in doped graphene, but only at $q=0$ relevant to Raman scattering experiments. Traditionally, KA is probed experimentally with X-ray or neutron scattering spectroscopy by measuring the phonon energy as a function of $q$, for which a systematic theory for the phonon energy renormalization in graphene for finite wavevector $q>0$ 
is still lacking.

In this chapter, we formulate a theory for the renormalization of the phonon energy dispersion in graphene due to both Coulomb and electron-phonon interaction

effects. From our theory, we obtain two major new results: (1) We find that direct Coulomb and phonon-mediated electron-electron interactions decouple to all orders of perturbation theory within RPA, and the electronic collective plasmon mode does not contribute to the phonon energy renormalization; (2) we obtain the renormalized phonon energy dispersion as a function of $q$, predicting the occurrence of three distinct KAs at the phonon wavevector $q=\omega / v, 2 k_{F} \pm \omega / v$ for the LO mode and one at $q=\omega / v$ for the TO mode, which arise from the chiral structure of the graphene electron-phonon coupling. The novel pecularity that these KAs do not occur at $q=$ $2 k_{F}$ (as in usual metals) originates from the fact that the phonon dynamics cannot be neglected in the screened electron-phonon interaction, indicating the inapplicability of the BA.

\subsection{Validity of the Born-Oppenheimer}

\section{Approximation}

A wider perspective of the applicability of the BO approximation is in order. In metals, the phonon energy renormalization is usually considered within the adiabatic BO approximation, which is equivalent to taking the phonon self-energy in the static limit. This static approximation is justified because in metals the Fermi energy $\varepsilon_{F}$ 
and the plasmon energy $\omega_{\mathrm{p}}$ are much larger than the Debye energy $\omega_{\mathrm{D}}$ for acoustic phonons (which are the only important type of phonons in metals), e.g., for sodium $\varepsilon_{F} \simeq 3 \mathrm{eV}, \omega_{\mathrm{p}} \simeq 6 \mathrm{eV} \gg \omega_{\mathrm{D}}=13 \mathrm{meV}$, with the lattice vibrational motion being essentially static compared with the electron motion. In two-dimensional systems such as doped semiconductors, $\varepsilon_{F}, \omega_{\mathrm{p}}$ are of the same order of magnitude as $\omega_{0}\left(\omega_{0}\right.$ is the optical phonon energy), e.g., for 2D GaAs at a density $n=10^{11}-10^{12} \mathrm{~cm}^{-2}$, $\varepsilon_{F} \simeq 3.6-36 \mathrm{meV}, \omega_{\mathrm{p}} \sim 30-160 \mathrm{meV}$ while $\omega_{0}=36.8 \mathrm{meV}$; the lattice dynamics is substantial compared with the electron motion and hence the static approximation fails.

Therefore, the $\mathrm{BO}$ approximation breaks down in usual doped semiconductors at low enough densities (i.e., when $\varepsilon_{F} \sim \omega_{0}$ ). In fact, it would be strictly incorrect to use the static (i.e., $\mathrm{BO}$ or adiabatic) approximation for the phonon degree of freedom in these systems. Within the static approximation, no plasmon-phonon coupling can occur because the two modes are effectively decoupled; whereas in reality coupled plasmon-phonon modes do occur [74] due to the hybridization of the plasmon and phonon modes, a phenomenon which can only be described correctly by incorporating the full dynamical screening of Coulomb and electron-phonon interactions. In this vein, we emphasize that the validity of the $\mathrm{BO}$ approximation should not be taken as granted, and the adiabatic condition $\omega_{0} / \varepsilon_{F} \ll 1$ for which the approximation is justified should always be kept in mind.

Graphene, behaving as a 2D zero-gap semiconductor, has a G-band optical phonon energy $\omega_{0}=200 \mathrm{meV}$ at the $\Gamma$ point which is comparable to the Fermi en$\operatorname{ergy} \varepsilon_{F} \sim 110-370 \mathrm{meV}$ at the usual extrinsic carrier density $n=10^{12}-10^{13} \mathrm{~cm}^{-2}$. 
This approximate equality of the phonon and electron energy scales $\omega_{0} \sim \varepsilon_{F}$ implies a breakdown of the static approximation for the phonon degree of freedom, naturally explaining the violation of the $\mathrm{BO}$ approximation in the recent Raman scattering experiments $[18,60,61,62]$ since the $\mathrm{BO}$ approximation implicitly assumes the phonon dynamics to be much slower than the electron dynamics. In addition, plasmon-phonon coupling, which has been extensively studied in doped semiconductor systems (e.g., GaAs, $\mathrm{SiC}$ ), is expected to occur whenever the phonon dynamics is at a comparable time scale as the electron motion. It follows that one cannot take the screening of the electron-phonon interaction to be simply static while keeping the screening of the Coulomb interaction to be dynamic; instead, one has to take into account the dynamical screening of both Coulomb and electronphonon interactions, i.e., direct electron-electron and electron-phonon interactions must be treated on an equal footing [74].

\subsection{Formalism}

In the presence of direct electron-electron interaction and electron-phonon interaction, the phonon Green function is renormalized by both, as represented in the diagrammatic language in Fig. 5.1. Summation of these diagrams leads to the renormalized phonon Green function $\mathcal{D}(q, \omega)=\mathcal{D}^{0}(q, \omega) /\left[1-\mathcal{D}^{0}(q, \omega) \Pi^{\mathrm{pp}}(q, \omega)\right]$, where $\mathcal{D}^{0}(q, \omega)=2 \omega_{0} /\left(\omega^{2}-\omega_{0}^{2}+i 0^{+}\right)$is the bare phonon Green function with $\omega_{0}$ the optical phonon energy, $\Pi^{\mathrm{pp}}(q, \omega)$ is the RPA-screened phonon self-energy given by 


\section{$m m=m+M m+N$}

(a)

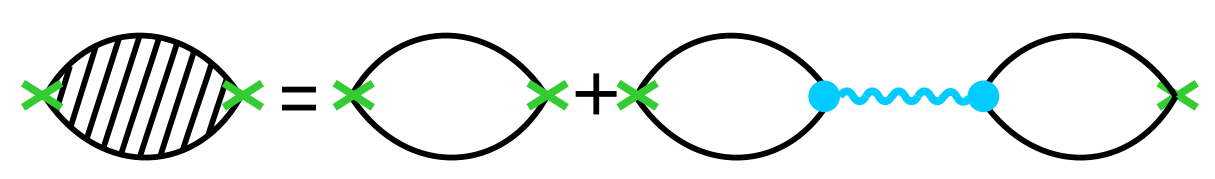

(b)

Figure 5.1: (a) Dyson equation for the renormalization of the phonon Green function. The zigzag lines denote the phonon Green function and the crosses denote the electron-phonon interaction vertex. The shaded bubble with two cross vertices stands for the renormalized phonon self-energy. (b) Equation for the renormalized phonon self-energy. The unshaded bubble with two cross vertices denotes the bare phonon self-energy, the two bubbles with one cross vertex and one dot vertex are "hybrid bubbles" with one electron-phonon interaction vertex and one Coulomb interaction vertex. The wavy line stands for the usual RPA-screened Coulomb interaction.

the following equation [Fig. 5.1(b)]:

$$
\Pi^{\mathrm{pp}}(q, \omega)=\Pi_{0}^{\mathrm{pp}}(q, \omega)+\Pi_{0}^{\mathrm{pc}}(q, \omega) V_{\mathrm{ee}}^{\mathrm{c}}(q, \omega) \Pi_{0}^{\mathrm{cp}}(q, \omega),
$$

where $V_{\mathrm{ee}}^{\mathrm{c}}(q, \omega)=V(q) /\left[1-V(q) \Pi_{0}^{\mathrm{cc}}(q, \omega)\right]$ is the usual RPA-screened Coulomb interaction (c.f. Chapter 2), $\Pi_{0}^{\mathrm{cc}}(q, \omega)$ is the irreducible polarizability (we have restored the subscript ' 0 ' emphasizing it is a "bare" bubble), $\Pi_{0}^{\mathrm{pp}}(q, \omega)$ the bare phonon self-energy, and $\Pi_{0}^{\mathrm{pc}}(q, \omega), \Pi_{0}^{\mathrm{cp}}(q, \omega)$ are the bare "hybrid" bubbles with one Coulomb interaction vertex and one electron-phonon interaction vertex. The superscripts 'p' denotes an electron-phonon interaction vertex whereas 'c' denotes a Coulomb interaction vertex. In regular metals or doped polar semiconductors, the 
phonon self-energy and the hydrid bubbles are the same (up to a factor given by the electron-phonon coupling constant) as the electronic polarizability; the Dyson equation for the renormalized phonon Green function (Fig. 5.1) simply reduces to the usual RPA series. In graphene, due to the presence of a chiral structure of the electron-phonon interaction Eq. (4.3), the bubbles $\Pi_{0}^{\mathrm{pp}}, \Pi_{0}^{\mathrm{pc}}, \Pi_{0}^{\mathrm{cp}}$, and $\Pi_{0}^{\mathrm{cc}}$ are not equal to one another. In particular, $\Pi_{0}^{\mathrm{pc}}$ and $\Pi_{0}^{\mathrm{cp}}$ incorporate the coupling effects of the Coulomb and electron-phonon interactions within the RPA, and describe the renormalization effect of the phonon energy by the Coulomb interaction. Interestingly, we find that with the graphene electron-phonon interaction Eq. (4.3), these hybrid bubbles vanish identically: $\Pi_{0}^{\mathrm{pc}}=\Pi_{0}^{\mathrm{cp}}=k_{\mathrm{B}} T g \sum_{i k_{n}, k} G_{k \lambda}^{0}\left(i k_{n}\right) G_{k+q \lambda^{\prime}}^{0}\left(i k_{n}+\right.$ $\left.i q_{n}\right)\left\langle\mathbf{k}+\mathbf{q} \lambda^{\prime}|\mathbf{M}(\mathbf{q})| \mathbf{k} \lambda\right\rangle\left\langle\mathbf{k} \lambda \mid \mathbf{k}+\mathbf{q} \lambda^{\prime}\right\rangle=\mathbf{0}$, Eq. (5.1) then implies that $\Pi^{\mathrm{pp}}$ is simply given by the bare phonon self-energy $\Pi_{0}^{\mathrm{pp}}$, and Coulomb interaction does not contribute to the screening of the electron-phonon interaction within the RPA. The phonon energy dispersion, which is given by the pole of the real part of the renormalized phonon Green function,

$$
\omega^{2}=\omega_{0}^{2}+2 \omega_{0} \operatorname{Re} \Pi_{0}^{\mathrm{pp}}(q, \omega)
$$

is therefore only renormalized by the electron-phonon interaction but not by Coulomb interaction. It follows that coupled plasmon-phonon modes do not arise in graphene, with the phonon and plasmon modes having separate branches of energy dispersion in the $\omega-q$ phase space despite comparable energy scales for the phonon and electron dynamics. In addition, direct Coulomb and phonon-mediated electron-electron 
interactions are simply additive, with the RPA-screened total electron-electron interaction given by

$$
V_{\mathrm{ee}}^{\mathrm{tot}}=\frac{V(q)}{1-V(q) \Pi_{0}^{\mathrm{cc}}}+\frac{V_{\mathrm{ee}}^{\mathrm{ph}}}{1-\mathcal{D}^{0} \Pi_{0}^{\mathrm{pp}}},
$$

where $V_{\mathrm{ee}}^{\mathrm{ph}}$ is the unscreened phonon-mediated electron-electron interaction (c.f. Eq. (4.10)). This decoupling is consistent with the physical picture that graphene is non-polar and optical phonons are due to bond stretching and bending, instead of Coulomb interaction between free carriers and polar atoms as in a polar semiconductor.

\subsection{Phonon self-energy}

The phonon self-energy $\Pi^{\mathrm{pp}}=\Pi_{0}^{\mathrm{pp}}$ can then be derived from the electronphonon interaction vertex Eq. 4.3 as:

$$
\begin{aligned}
\Pi^{\mathrm{pp}}\left(q, i q_{n}\right)= & 4 g^{2} k_{\mathrm{B}} T \sum_{\lambda \lambda^{\prime}} \sum_{k, i k_{n}} G_{k+q \lambda^{\prime}}\left(i k_{n}+i q_{n}\right) G_{k \lambda}\left(i k_{n}\right) \\
& \left\langle\boldsymbol{k}+\boldsymbol{q} \lambda^{\prime}|M(\boldsymbol{q})| \boldsymbol{k} \lambda\right\rangle\left\langle\boldsymbol{k} \lambda|M(-\boldsymbol{q})| \boldsymbol{k}+\boldsymbol{q} \lambda^{\prime}\right\rangle \\
= & 4 g^{2} k_{\mathrm{B}} T \sum_{\lambda \lambda^{\prime}} \sum_{k, i k_{n}} G_{k+q \lambda^{\prime}}\left(i k_{n}+i q_{n}\right) G_{k \lambda}\left(i k_{n}\right) \frac{1 \mp \lambda \lambda^{\prime} \cos \left(\phi_{k+q}+\phi_{k}\right)}{2},
\end{aligned}
$$

where the sign $-(+)$ corresponds to $\mathrm{LO}(\mathrm{TO})$ phonons, the factor of 4 counts the spin and valley degeneracies, and $\phi_{k}=\tan ^{-1}\left(k_{y} / k_{x}\right)$ is the azithmuthal angle of the momentum $\mathbf{k}$ measured from $\mathbf{q}$. Carrying out the Matsubara sum over $i k_{n}$, we obtain

$$
\Pi^{\mathrm{pp}}\left(q, i q_{n}\right)=4 g^{2} \sum_{\lambda \lambda^{\prime}} \sum_{k} \frac{n_{F}\left(\xi_{k \lambda}\right)-n_{F}\left(\xi_{k+q \lambda^{\prime}}\right)}{i q_{n}+\xi_{k \lambda}-\xi_{k+q \lambda^{\prime}}} \frac{1 \mp \lambda \lambda^{\prime} \cos \left(\phi_{k+q}+\phi_{k}\right)}{2} .
$$


We are interested in the real part of the phonon self-energy which renormalizes the phonon energy dispersion. Taking the real part of the phonon self-energy in Eq. (5.5) and dropping the notation 'Re' for the real part thereafter (understanding only the real part is of interest in the rest of this chapter), the phonon self-energy can be written from Eq. (5.5) as

$$
\begin{aligned}
& \Pi^{\mathrm{pp}}(q, \omega)=2 g^{2} \sum_{\boldsymbol{k}}\left\{n_{F}\left(\xi_{\boldsymbol{k}+}\right)\left[\frac{1 \mp \cos \left(\phi_{k+q}+\phi_{k}\right)}{\omega+v k-v|\boldsymbol{k}+\boldsymbol{q}|}+\frac{1 \pm \cos \left(\phi_{k+q}+\phi_{k}\right)}{\omega+v k+v|\boldsymbol{k}+\boldsymbol{q}|}\right]\right. \\
& -n_{F}\left(\xi_{\boldsymbol{k}+\boldsymbol{q}+}\right)\left[\frac{1 \mp \cos \left(\phi_{k+q}+\phi_{k}\right)}{\omega-v|\boldsymbol{k}+\boldsymbol{q}|+v k}+\frac{1 \pm \cos \left(\phi_{k+q}+\phi_{k}\right)}{\omega-v|\boldsymbol{k}+\boldsymbol{q}|-k}\right] \\
& +n_{F}\left(\xi_{\boldsymbol{k}-}\right)\left[\frac{1 \mp \cos \left(\phi_{k+q}+\phi_{k}\right)}{\omega-v k+v|\boldsymbol{k}+\boldsymbol{q}|}+\frac{1 \pm \cos \left(\phi_{k+q}+\phi_{k}\right)}{\omega-v k-v|\boldsymbol{k}+\boldsymbol{q}|}\right] \\
& \left.-n_{F}\left(\xi_{\boldsymbol{k}+\boldsymbol{q}-}\right)\left[\frac{1 \mp \cos \left(\phi_{k+q}+\phi_{k}\right)}{\omega+v|\boldsymbol{k}+\boldsymbol{q}|-v k}+\frac{1 \pm \cos \left(\phi_{k+q}+\phi_{k}\right)}{\omega+v|\boldsymbol{k}+\boldsymbol{q}|+v k}\right]\right\} .
\end{aligned}
$$

From Eq. (5.6), we see that the real part of the phonon self-energy comprises two contributions $\Pi_{\mathrm{LO}, \mathrm{TO}}^{\mathrm{pp}} \equiv \Pi_{\mathrm{LO}, \mathrm{TO}+}^{\mathrm{pp}}+\Pi_{\mathrm{LO}, \mathrm{TO}-}^{\mathrm{pp}}$, where

$$
\Pi_{\mathrm{LO}, \mathrm{TO} \mu}^{\mathrm{pp}}=2 g^{2} \sum_{\lambda} \sum_{k} n_{F}\left(\xi_{k \mu}\right) f_{\mu \lambda}(\boldsymbol{k}, \boldsymbol{q}),
$$

with

$$
f_{\mu \lambda}(\boldsymbol{k}, \boldsymbol{q})=\frac{1 \mp \lambda \cos \left(\phi_{k+q}+\phi_{k}\right)}{\omega+\mu \xi_{k+}-\xi_{k+q \lambda}}-\frac{1 \mp \lambda \cos \left(\phi_{k}+\phi_{k-q}\right)}{\omega-\mu \xi_{k+}+\xi_{k-q \lambda}} .
$$

Eq. (5.6) can be simplified as

$$
\begin{aligned}
& \Pi^{\mathrm{pp}}(q, \omega)=g^{2} \sum_{\boldsymbol{k}}\{ \\
& n_{F}\left(\xi_{\boldsymbol{k}+}\right)\left[\frac{\omega+v k \mp v|\boldsymbol{k}+\boldsymbol{q}| \cos \left(\phi_{k+q}+\phi_{k}\right)}{(\omega+v k)^{2}-v^{2}|\boldsymbol{k}+\boldsymbol{q}|^{2}}-\frac{\omega-v k \pm v|\boldsymbol{k}-\boldsymbol{q}| \cos \left(\phi_{k}+\phi_{k-q}\right)}{(\omega-v k)^{2}-v^{2}|\boldsymbol{k}-\boldsymbol{q}|^{2}}\right] \\
& \left.+n_{F}\left(\xi_{\boldsymbol{k}-}\right)\left[\frac{\omega-v k \pm v|\boldsymbol{k}+\boldsymbol{q}| \cos \left(\phi_{k+q}+\phi_{k}\right)}{(\omega-v k)^{2}-v^{2}|\boldsymbol{k}+\boldsymbol{q}|^{2}}-\frac{\omega+v k \mp v|\boldsymbol{k}-\boldsymbol{q}| \cos \left(\phi_{k}+\phi_{k-q}\right)}{(\omega+v k)^{2}-v^{2}|\boldsymbol{k}-\boldsymbol{q}|^{2}}\right]\right\} .
\end{aligned}
$$


Using the trigonometric relations $|\boldsymbol{k} \pm \boldsymbol{q}| \cos \left(\phi_{k \pm q}+\phi_{k}\right)=-k+2 k^{2} \cos ^{2} \phi_{k} \pm q \cos \phi_{k}$, we can write, for the LO mode,

$$
\begin{aligned}
& \Pi_{\mathrm{LO}}^{\mathrm{pp}}(q, \omega)=g^{2} \sum_{\boldsymbol{k}}\{ \\
& n_{F}\left(\xi_{\boldsymbol{k}+}\right)\left[\frac{\omega+2 v k-v q \cos \phi_{k}-2 v k \cos ^{2} \phi_{k}}{(\omega+v k)^{2}-v^{2}|\boldsymbol{k}+\boldsymbol{q}|^{2}}-\frac{\omega-2 v k-v q \cos \phi_{k}+2 v k \cos ^{2} \phi_{k}}{(\omega-v k)^{2}-v^{2}|\boldsymbol{k}-\boldsymbol{q}|^{2}}\right] \\
& \left.+n_{F}\left(\xi_{\boldsymbol{k}-}\right)\left[\frac{\omega-2 v k+v q \cos \phi_{k}+2 v k \cos ^{2} \phi_{k}}{(\omega-v k)^{2}-v^{2}|\boldsymbol{k}+\boldsymbol{q}|^{2}}-\frac{\omega+2 v k+v q \cos \phi_{k}-2 v k \cos ^{2} \phi_{k}}{(\omega+v k)^{2}-v^{2}|\boldsymbol{k}-\boldsymbol{q}|^{2}}\right]\right\},
\end{aligned}
$$

and for the TO mode,

$$
\begin{aligned}
& \Pi_{\mathrm{TO}}^{\mathrm{pp}}(q, \omega)=g^{2} \sum_{\boldsymbol{k}}\{ \\
& n_{F}\left(\xi_{\boldsymbol{k}+}\right)\left[\frac{\omega+v q \cos \phi_{k}+2 v k \cos ^{2} \phi_{k}}{(\omega+v k)^{2}-v^{2}|\boldsymbol{k}+\boldsymbol{q}|^{2}}-\frac{\omega+v q \cos \phi_{k}-2 v k \cos ^{2} \phi_{k}}{(\omega-v k)^{2}-v^{2}|\boldsymbol{k}-\boldsymbol{q}|^{2}}\right] \\
& \left.+n_{F}\left(\xi_{\boldsymbol{k}-}\right)\left[\frac{\omega-v q \cos \phi_{k}-2 v k \cos ^{2} \phi_{k}}{(\omega-v k)^{2}-v^{2}|\boldsymbol{k}+\boldsymbol{q}|^{2}}-\frac{\omega-v q \cos \phi_{k}+2 v k \cos ^{2} \phi_{k}}{(\omega+v k)^{2}-v^{2}|\boldsymbol{k}-\boldsymbol{q}|^{2}}\right]\right\} .
\end{aligned}
$$

The contribution $\Pi_{+}^{\mathrm{pp}}$ is due to the extrinsic conduction band electrons whereas $\Pi_{-}^{\mathrm{pp}}$ is solely due to the intrinsic valence band electrons. In the renormalization of the phonon energy in extrinsic graphene, $\Pi_{-}^{\mathrm{pp}}$ should be subtracted from the total $\Pi^{\mathrm{pp}}$ to avoid overcounting of the intrinsic contribution, since the "bare" phonon energy (i.e. when graphene is undoped), by definition, already includes the effect of $\Pi_{-}^{\mathrm{pp}}[19,20]$. Therefore, only $\Pi_{+}^{\mathrm{pp}}$ should be taken into account in the phonon energy renormalization by free carriers in extrinsic graphene. We also note that, for $\Pi_{+}^{\mathrm{pp}}$, the largest energy scale is the Fermi energy $\varepsilon_{F}$ whereas for $\Pi_{-}^{\mathrm{pp}}$ it is much higher, of the order of the cutoff energy $\Lambda_{c}=2 \pi \hbar v / a$ for the graphene linear band dispersion ( $a=2.46 \AA$ is the graphene lattice spacing). Therefore, although the BA 
is inapplicable for extrinsic graphene with $\omega_{0} / \varepsilon_{F} \sim \mathcal{O}(1)$, for intrinsic graphene the $\mathrm{BA}$ is strictly valid since $\omega_{0} / \Lambda_{c} \ll 1$.

We can then write $\Pi_{\mathrm{LO}+}^{\mathrm{pp}}$ for the LO mode from Eq. (5.10) as

$$
\begin{aligned}
\Pi_{\mathrm{LO}+}^{\mathrm{pp}}(q, \omega)= & \frac{g^{2} k_{F}}{(2 \pi)^{2} v} \int_{0}^{1} k \mathrm{~d} k \int_{0}^{2 \pi} \mathrm{d} \phi_{k}\left\{\frac{1}{k}\left[1+\frac{1}{2}\left(\frac{b_{+}-a_{+}}{a_{+}-\cos \phi_{k}}-\frac{b_{-}+a_{-}}{a_{-}+\cos \phi_{k}}\right)\right]\right. \\
& \left.-\frac{1}{q} \cos ^{2} \phi_{k}\left(\frac{1}{a_{+}-\cos \phi_{k}}+\frac{1}{a_{-}+\cos \phi_{k}}\right)\right\}
\end{aligned}
$$

and $\Pi_{\mathrm{TO}+}^{\mathrm{pp}}$ for the TO mode from Eq. (5.11) as

$$
\begin{aligned}
\Pi_{\mathrm{TO}+}^{\mathrm{pp}}(q, \omega)= & \frac{g^{2} k_{F}}{(2 \pi)^{2} v} \int_{0}^{1} k \mathrm{~d} k \int_{0}^{2 \pi} \mathrm{d} \phi_{k}\left\{-\frac{1}{k}\left[1-\frac{1}{2}\left(\frac{b+a_{+}}{a_{+}-\cos \phi_{k}}+\frac{b-a_{-}}{a_{-}+\cos \phi_{k}}\right)\right]\right. \\
& \left.+\frac{1}{q} \cos ^{2} \phi_{k}\left(\frac{1}{a_{+}-\cos \phi_{k}}+\frac{1}{a_{-}+\cos \phi_{k}}\right)\right\}
\end{aligned}
$$

where in Eqs. (5.12)-(5.13) we have defined

$$
\begin{aligned}
& a_{ \pm}=\frac{(\omega / v)^{2}-q^{2} \pm 2 \omega k / v}{2 k q} \\
& b_{ \pm}=\frac{\omega / v \pm 2 k}{q} \\
& b=\frac{\omega}{v q}
\end{aligned}
$$

Using the integral identity

$$
\int_{0}^{2 \pi} \mathrm{d} \phi \frac{1}{a \pm b \cos \phi}=\frac{2 \pi}{\sqrt{a^{2}-b^{2}}} \operatorname{sgn}(a \pm b)
$$

the angular integrals in Eqs. (5.12)-(5.13) can be carried out, giving

$$
\begin{aligned}
& \tilde{\Pi}_{\mathrm{LO}+}^{\mathrm{pp}}(q, \omega)=\int_{0}^{1} \mathrm{~d} k\left\{1+\frac{1}{2}\left[\frac{b_{+}-a_{+}}{\sqrt{a_{+}^{2}-1}} \operatorname{sgn}\left(a_{+}-1\right)-\frac{b_{-}+a_{-}}{\sqrt{a_{-}^{2}-1}} \operatorname{sgn}\left(a_{-}+1\right)\right]\right\} \\
& -\frac{1}{q} \int_{0}^{1} \mathrm{~d} k k\left\{a_{+}\left[\frac{a_{+}}{\sqrt{a_{+}^{2}-1}} \operatorname{sgn}\left(a_{+}-1\right)-1\right]+a_{-}\left[\frac{a_{-}}{\sqrt{a_{-}^{2}-1}} \operatorname{sgn}\left(a_{-}+1\right)-1\right]\right\},
\end{aligned}
$$


and

$$
\begin{aligned}
& \tilde{\Pi}_{\mathrm{TO}+}^{\mathrm{pp}}(q, \omega)=\int_{0}^{1} \mathrm{~d} k\left\{-1+\frac{1}{2}\left[\frac{b+a_{+}}{\sqrt{a_{+}^{2}-1}} \operatorname{sgn}\left(a_{+}-1\right)-\frac{b-a_{-}}{\sqrt{a_{-}^{2}-1}} \operatorname{sgn}\left(a_{-}+1\right)\right]\right\} \\
& +\frac{1}{q} \int_{0}^{1} \mathrm{~d} k k\left\{a_{+}\left[\frac{a_{+}}{\sqrt{a_{+}^{2}-1}} \operatorname{sgn}\left(a_{+}-1\right)-1\right]+a_{-}\left[\frac{a_{-}}{\sqrt{a_{-}^{2}-1}} \operatorname{sgn}\left(a_{-}+1\right)-1\right]\right\},
\end{aligned}
$$

where we have expressed the phonon self-energy in Eqs. (5.16)-(5.17) in dimensionless units: $\tilde{\Pi}^{\mathrm{pp}}=\Pi^{\mathrm{pp}} /\left(2 g_{\mathrm{ee}}^{2} \varepsilon_{\mathrm{F}} / \pi\right)$ with $g_{\mathrm{ee}}^{2}=g^{2} \mathcal{A} / \hbar^{2} v^{2}$ the dimensionless phononmediated electron-electron coupling constant ( $\mathcal{A}$ is the sample area). The integrals over $k$ in Eqs. (5.16)-(5.17) can then performed in an exact analytical fashion, however extreme care needs to be exercised for the integration limits imposed by the square roots and the sign functions. First, simpler results can be obtained by considering the two limiting cases: long wavelength limit $q \ll k_{F}$ and static limit $\omega=0$.

\subsubsection{Long-wavelength limit}

For clarity, we express our results in the following dimensionless quantities: wavevector $x=q / k_{F}$, energy $u=\omega / \varepsilon_{F}$. We have obtained the following asymptotic results for the phonon self-energy in the limit $q / k_{F} \ll 1$ :

$$
\tilde{\Pi}_{\mathrm{LO}, \mathrm{TO}+}^{\mathrm{pp}}(x, u)=\frac{1}{2}\left[1+\frac{u}{4} \ln \left|\frac{2-u}{2+u}\right|\right]+\Delta \tilde{\Pi}_{\mathrm{LO}, \mathrm{TO}+}^{\mathrm{pp}}(x, u),
$$

where the next order correction to the long wavelength result is given by

$$
\begin{aligned}
& \Delta \tilde{\Pi}_{\mathrm{LO}+}^{\mathrm{pp}}(x, u)=\frac{1}{2}\left[\frac{8-4 u^{2}-u^{4}}{2 u^{2}\left(u^{2}-4\right)^{2}}-\frac{1}{8 u} \ln \left|\frac{2-u}{2+u}\right|\right] x^{2}, \\
& \Delta \tilde{\Pi}_{\mathrm{TO}+}^{\mathrm{pp}}(x, u)=\frac{1}{2}\left[\frac{24-12 u^{2}+u^{4}}{2 u^{2}\left(u^{2}-4\right)^{2}}+\frac{1}{8 u} \ln \left|\frac{2-u}{2+u}\right|\right] x^{2} .
\end{aligned}
$$


At $x=0$, the long wavelength result $[18,63,75]$ is the same for LO and TO phonons, the two phonon modes being degenerate at the $\Gamma$ point. At finite wavevector $x$, this degeneracy is lifted with the leading-order correction going as $x^{2}$ given by Eqs. (5.19)-(5.20).

\subsubsection{Static limit}

We have also obtained the following analytic results for the phonon self-energy in the static case $u=0$ :

$$
\begin{aligned}
\tilde{\Pi}_{\mathrm{TO}+}^{\mathrm{pp}}(x, 0)= & 0 \\
\tilde{\Pi}_{\mathrm{LO}+}^{\mathrm{pp}}(x, 0)= & -(\pi / 8) x \theta(2-x)+(1 / 4)\left[(2 / x) \sqrt{x^{2}-4}\right. \\
& \left.-x \tan ^{-1}\left(2 / \sqrt{x^{2}-4}\right)\right] \theta(x-2) .
\end{aligned}
$$

The above static phonon self-energy results are depicted in the inset of Fig. 5.2, which clearly shows the presence of a non-analyticity at $q=2 k_{F}$ corresponding to the KA for LO phonons. We also note that this non-analyticity is entirely absent in the static electronic polarizability [6] of graphene (Fig. 5.3). This is in contrast to the situation for regular materials with a parabolic energy dispersion where the phonon self-energy and the polarizability are equal up to a factor given by the electronphonon coupling. This distinctive difference between the phonon self-energy and the polarizability in graphene is a direct result of the presence of a chiral structure in the graphene electron-phonon coupling Eq. (4.3), which leads to a different Berry phase dependence in the expression of the phonon self-energy Eq. (5.4) compared 


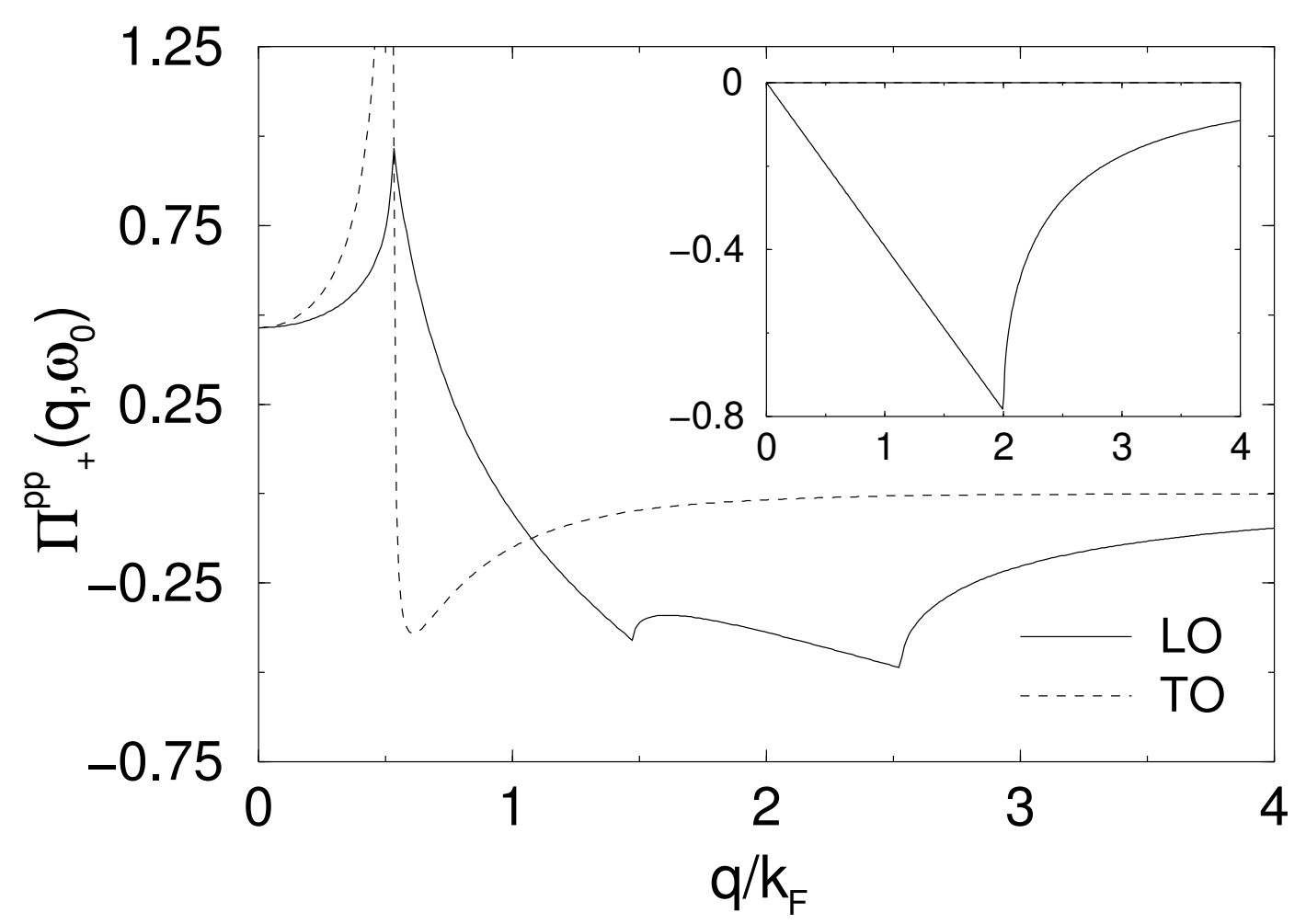

Figure 5.2: Dynamic phonon self-energy $\tilde{\Pi}_{+}^{\mathrm{pp}}(x, u)$ versus $x=q / k_{F}$ at $u=\omega_{0} / \varepsilon_{F}$ for the LO (solid line) and TO (dashed) modes at a density $n=10^{13} \mathrm{~cm}^{-2}$. Three cusps are apparent which correponds to the Kohn anomalies at $x=u, x=2 \pm u$ for LO phonons. For TO phonons, $\tilde{\Pi}_{+}^{\mathrm{pp}}$ diverges at $x=u$. Inset: Static phonon self-energy $\tilde{\Pi}_{+}^{\mathrm{pp}}(x, u)$ versus $x=q / k_{F}$ at $u=0$. $\tilde{\Pi}_{+}^{\mathrm{pp}}(x, 0)$ for the LO mode has a Kohn anomaly at $q=2 k_{F}$. $\tilde{\Pi}_{+}^{\mathrm{pp}}(x, 0)$ for the TO mode is zero.

with the polarizability Eq. (2.4). Therefore, KAs in graphene originate entirely from the special chiral structure of the electron-phonon coupling Eq. (4.3).

\subsubsection{Finite $q$ and $\omega$}

We have evaluated the full expression for $\Pi_{+}^{\mathrm{pp}}$ with general $q$ and $\omega$ dependence analytically (with numerical evaluation served as a consistency check), which is however too cumbersome to be shown here. The main plot of Fig. 5.2 shows the evaluated $\Pi_{+}^{\mathrm{pp}}$ as a function of $x$ at the phonon energy $\omega=\omega_{0}$, from which three cusps occurring at $v q=\omega_{0}, v q=2 \varepsilon_{F} \pm \omega_{0}$ for the LO mode are clearly discernible. 


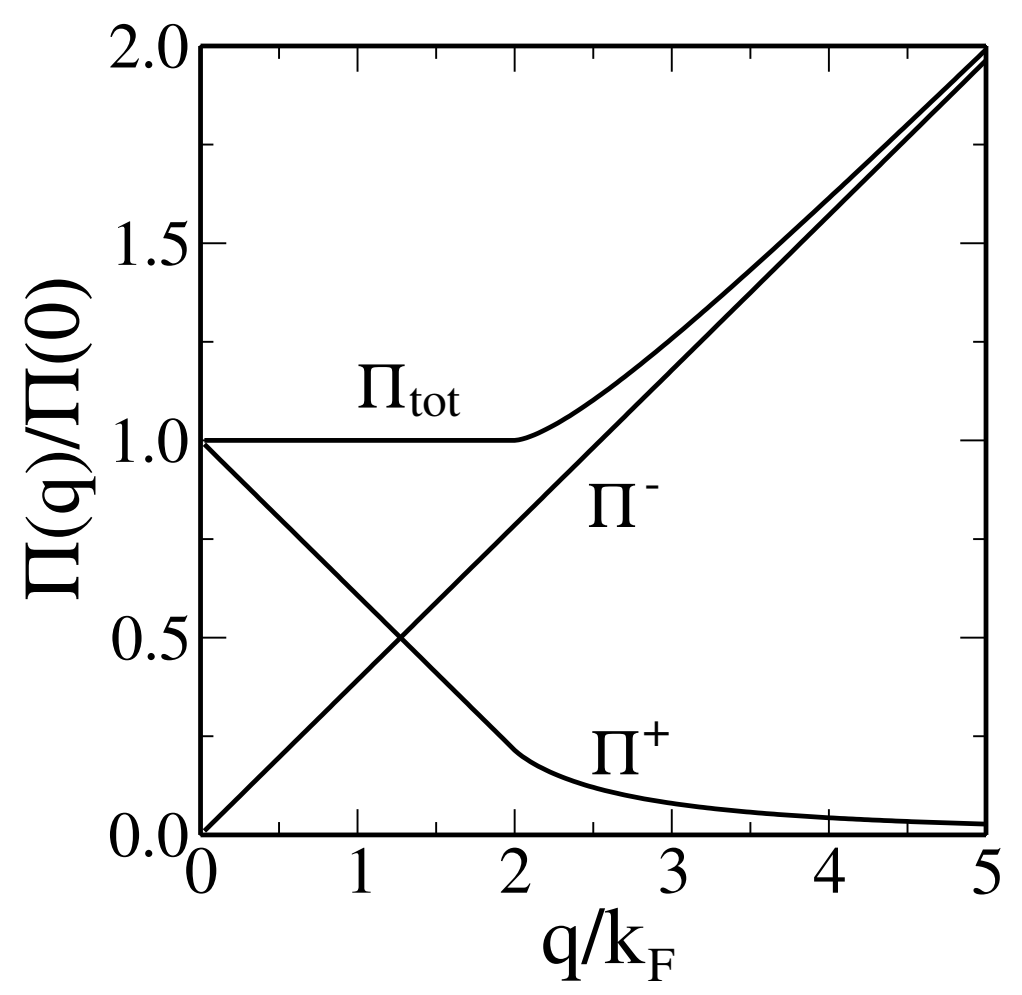

Figure 5.3: Static polarizability of graphene (adapted from Ref. [6]), with $\Pi_{\text {tot }}=\Pi_{0}^{\text {cc }}$ in the notation of this dissertation. $\Pi_{0}^{c c}$ does not show any cusp or kink structure for finite values of $q$.

For the TO mode, there is a divergence of $\Pi_{+}^{\mathrm{pp}}$ at $v q=\omega_{0}$. These non-analyticities correspond to the values of $q$ where the denominator of the integrand of $\Pi_{+}^{\mathrm{pp}}$ vanishes at the Fermi surface $k=k_{F}$, i.e. the zeros of the equation $\omega \pm v k_{F} \pm v\left|k_{F} \pm q\right|=0$.

\subsection{Renormalized phonon energy dispersion}

With the calculated phonon self-energy, the renormalized phonon energy spectrum can be obtained by self-consistently solving Eq. (5.2) for $\omega$, which is shown in Fig. 5.4 for the LO mode and Fig. 5.5 for the TO mode. For LO phonons, three KAs which correspond to the non-analyticities of $\Pi_{+}^{\mathrm{pp}}$ are evident, occurring at the wavevector $v q=\omega_{0}, v q=2 \varepsilon_{F} \pm \omega_{0}$. For TO phonons, the divergence of $\Pi_{+}^{\mathrm{pp}}$ at 


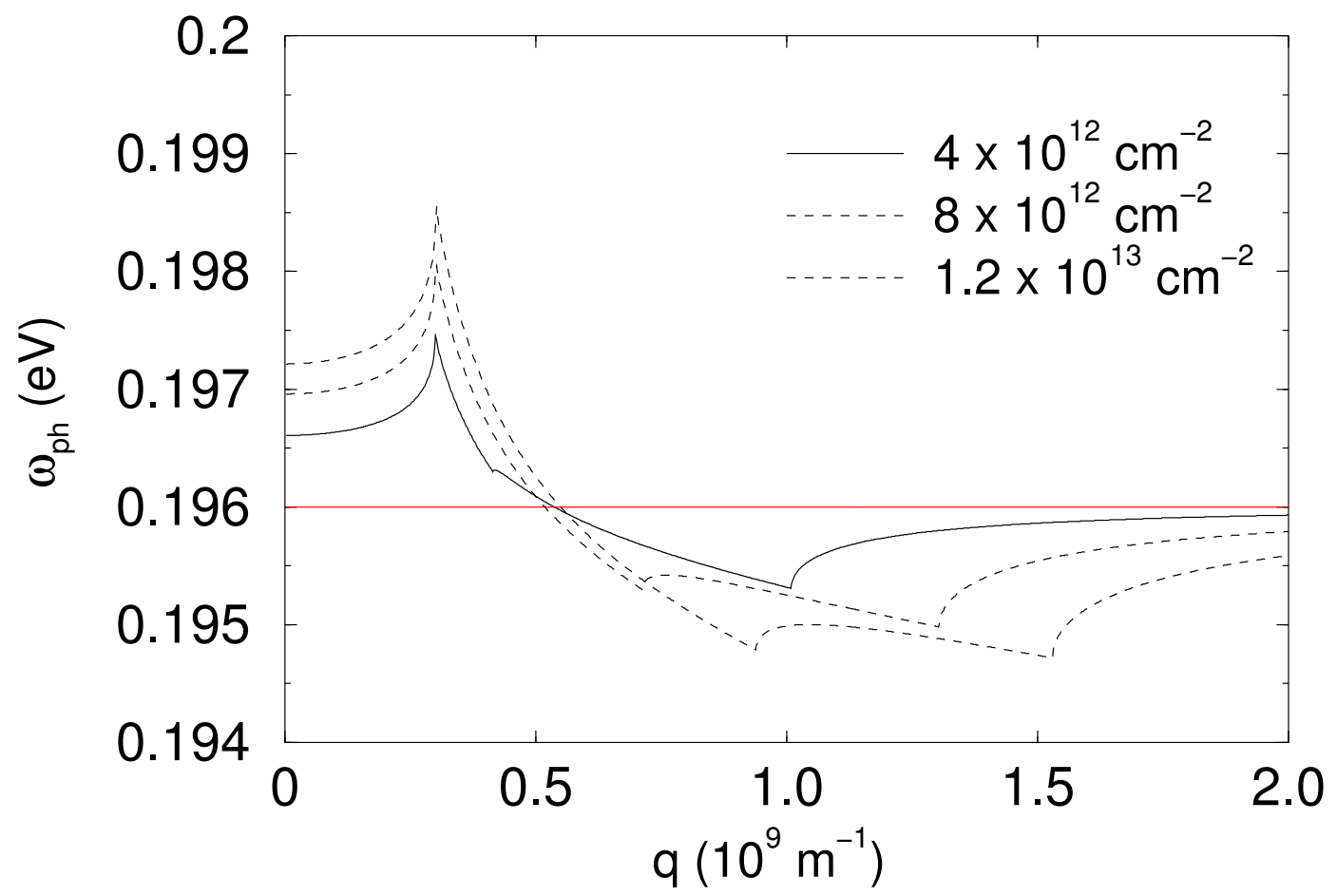

Figure 5.4: Renormalized LO phonon energy spectrum $\omega_{\text {ph }}$ versus $q$ at different electron densities $n$. The bare phonon energy is shown as the horizontal solid line. The range of phonon wavevector $q$ shown corresponds to $[0,0.08(2 \pi / a)]$ away from the $\Gamma$ point.

$v q=\omega_{0}$ is removed due to the self-consistency condition for $\omega$ in Eq. (5.2), but the KA remains as a sharp but finite peak at $v q=\omega_{0}$. In addition, we note the KA at $v q=\omega_{0}$ for both the $\mathrm{LO}$ and $\mathrm{TO}$ modes, unlike the other two KAs for the LO mode, is independent of electron density, an interesting consequence of the quasirelativistic linear dispersion peculiar to graphene. For both LO and TO phonons, our results suggest that the phonon energy first increases (i.e., phonon hardening) with density up to a certain phonon wavevector, and then decreases (i.e., phonon softening) with density. The critical wavevector for this transition from phonon hardening to softening is different for LO and TO phonons, and we find numerically $q \simeq 5 \times 10^{8} \mathrm{~m}^{-1}$ for LO phonons and $q=\omega_{0} / v=3 \times 10^{8} \mathrm{~m}^{-1}$ (i.e., the KA) for TO 


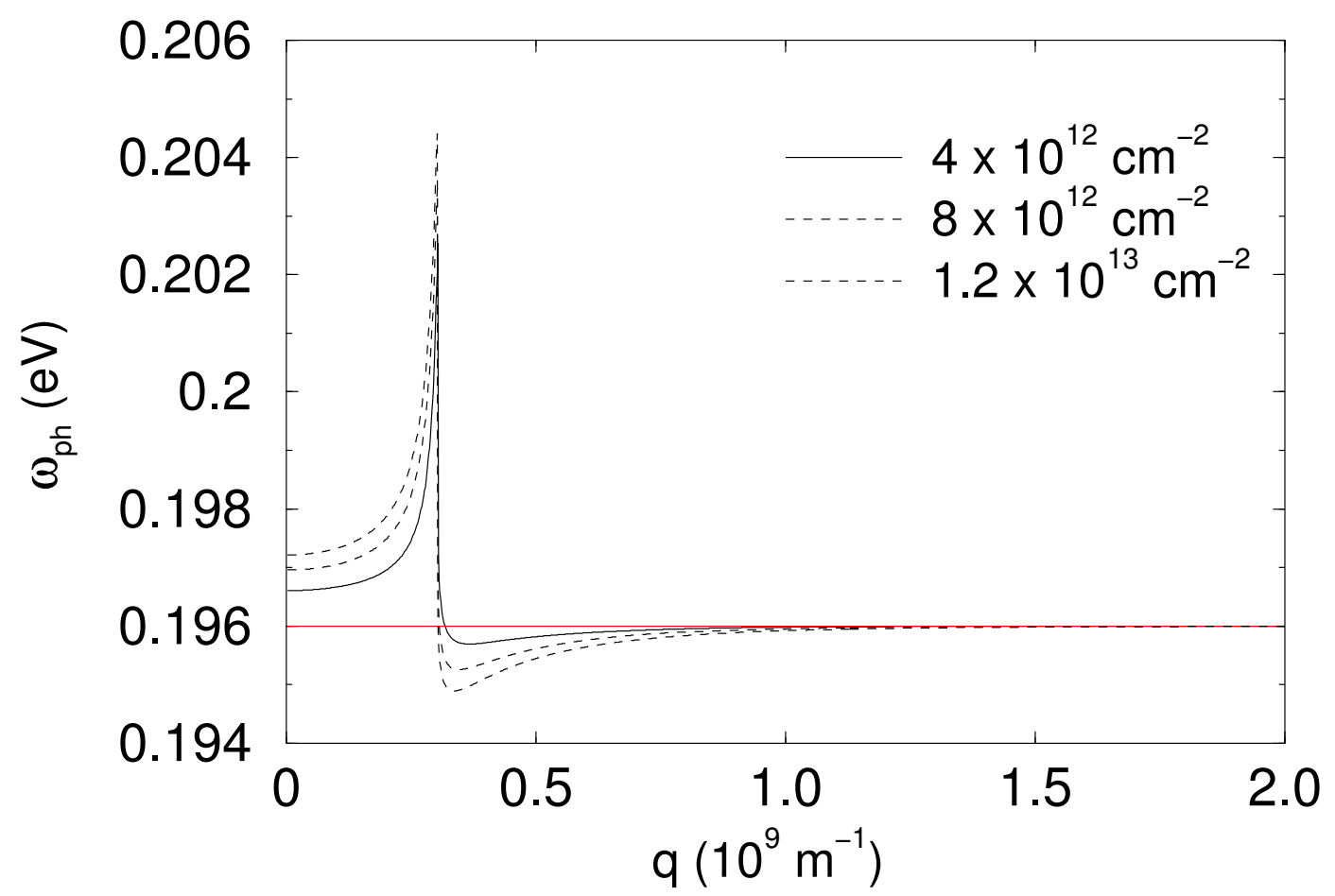

Figure 5.5: Renormalized TO phonon energy spectrum $\omega_{\text {ph }}$ versus $q$. The legends are the same as in Fig. 5.4.

phonons.

\subsection{Understanding the Kohn anomalies}

In the following, we provide a schematic picture for understanding the occurrence of the KAs. Fig. 5.6 shows the $\omega-q$ phase space in which single-particle excitation can occur through virtual phonon exchange. The behavior of the phonon self-energy is characterized by six different regions of the $\omega-q$ phase space where $\Pi_{+}^{\mathrm{pp}}$ is analytically continuous, separated by the boundaries (indicated by the solid lines) corresponding to the set of values of $(\omega, q)$ where $\Pi_{+}^{\mathrm{pp}}$ is non-analytic. Sufficient (but by no means necessary) conditions for the KAs to occur are given by the intersection points between the phonon dispersion line $\omega=\omega_{0}$ and the boundaries 


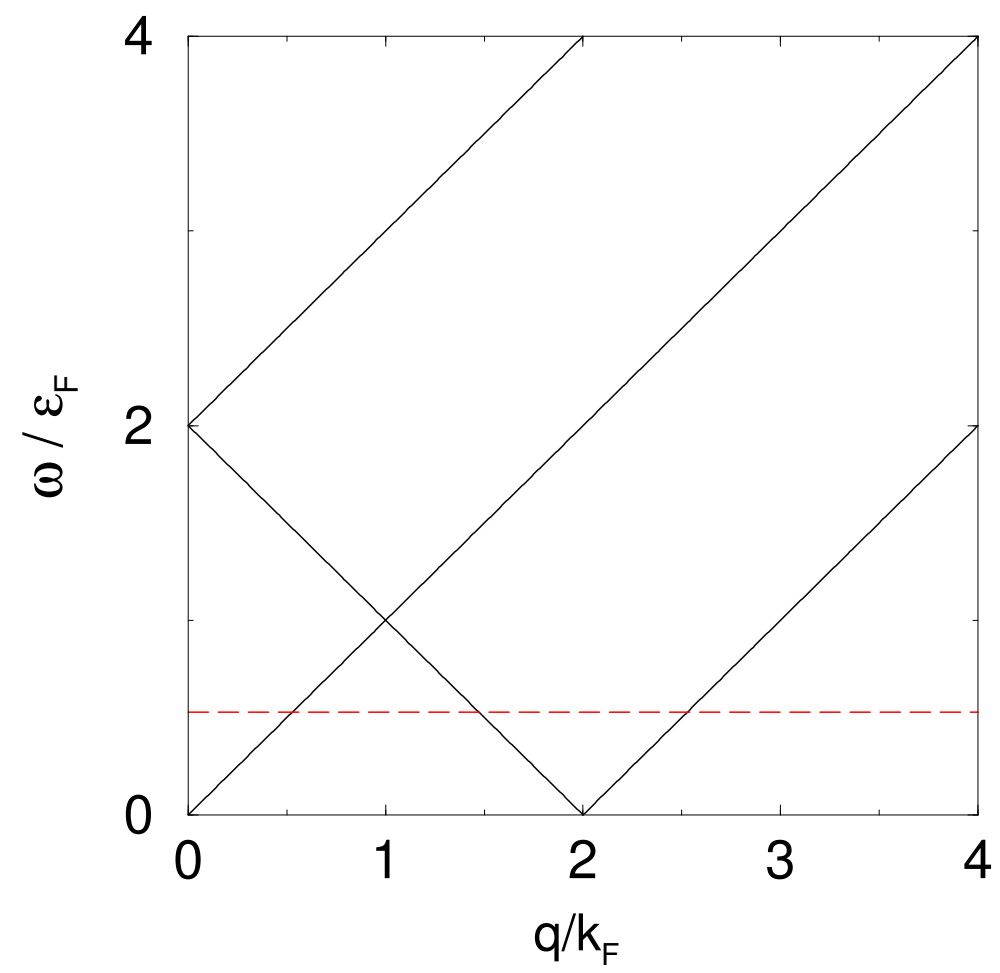

Figure 5.6: Different regions for the analytical behavior of $\Pi_{+}^{\mathrm{pp}}$. The solid lines indicate the boundaries for these regions, and the dashed line shows the phonon energy $\omega=\omega_{0}$ at a density $n=10^{13} \mathrm{~cm}^{-2}$.

for the different regions. Within the BA, phonons are treated as static with $\omega=0$ in the expression of the phonon self-energy, the only intersection points therefore occur at $q=0$ and $q=2 k_{F}$, as in the case of, e.g., a usual metal. In graphene where the BA is invalid, phonon dynamics must be included with $\omega=\omega_{0}$ in the phonon self-energy, the intersection points now occur at $v q=\omega_{0}, v q=2 \varepsilon_{F}-\omega_{0}$, and $v q=2 \varepsilon_{F}+\omega_{0}$. For the LO mode, we find that KAs occur at all three intersection points. This however does not apply for the TO mode, and we find two equal but opposite contributions in the expression for $\Pi_{\mathrm{TO}+}^{\mathrm{pp}}$ which cancel the effects of two KAs, yielding only one KA at $v q=\omega_{0}$ in this case. 
The above discussion applies to the case when $\varepsilon_{F}>\omega_{0} / 2$. With decreasing density (and therefore increasing $\omega_{0} / \varepsilon_{F}$ ), we note that the second KA for LO phonons at $v q=2 \varepsilon_{F}-\omega_{0}$ will go through a transition at $\varepsilon_{F}=\omega_{0} / 2$ to $v q=\omega_{0}-2 \varepsilon_{F}$ for $\varepsilon_{F} \leqslant \omega_{0} / 2$ (with the other two KAs remaining the same at $v q=\omega_{0}$ and $\left.v q=2 \varepsilon_{F}+\omega_{0}\right)$.

In conclusion, we have developed a theory for the interaction-induced phonon renormalization in graphene, and discovered completely new and multiple KAs in the renormalized phonon dispersion. The novel peculiarity and the remarkable distinction of these KAs from the usual KAs in metals are signatures of the dramatic nature of the renormalized dynamically screened electron-phonon interaction and the special chiral structure of the electron-phonon coupling in graphene. The experimental verification of our predictions would establish that graphene has a very unique electron-phonon many-body coupling. 


\section{Chapter 6}

\section{Conclusion and Outlook}

We first present a summary of our findings as a conclusion to the dissertation and then discuss open problems that can be pursued in the future. In the first Chapter, we presented a review of graphene physics, in particular derived the linear energy dispersion peculiar to graphene. We also discussed the experimental basis of angle-resolved photoemission spectroscopy (ARPES) and reviewed the important experimental findings in graphene ARPES experiments. In Chapter 2, we considered the effect of Coulomb electron-electron interaction on the quasiparticle properties, taking account of screening in the Random-Phase Approximation. We formulated the electron self-energy problem, and derived analytic expressions for the quasiparticle decay rate, renormalization factor and renormalized velocity for extrinsic graphene and intrinsic graphene. For extrinsic graphene, we found that the quasiparticle decay rate has a behavior characteristic of a two-dimensional Fermi liquid, going as $\sim \omega^{2} \ln \omega$; for intrinsic graphene, we found it goes as $\sim \omega$ signifying

the behavior of a marginal Fermi liquid. We conclude that any finite amount of doping will change the behavior of intrinsic graphene from a marginal Fermi liquid 
to a regular Fermi liquid, with the consequence that all existing experimental samples of graphene are regular Fermi liquid since doping will invariably be present. In Chapter 3, we presented a diagrammatic linear response theory for Coulomb drag in a double-layer graphene system. We showed that in any double-layer system where one layer is intrinsic graphene (whereas the other layer could be any kind of material), the drag resistivity is identically zero by virtue of electron-hole symmetry in the intrinsic graphene layer. In a double-layer system comprising two extrinsic graphene sheets, we found a finite drag resistivity as the electron-hole symmetry is already broken by the presence of a finite Fermi level. We derived an analytical result of the drag resistivity in the regime of low temperature, high density and/or large interlayer distance, finding it to be the same as in a double-layer system with regular parabolic dispersion. We also derived an exact analytical expression for the nonlinear susceptibility valid at zero temperature, and calculated the drag resistivity numerically as a function of temperature at different values of $k_{F} d$. We discussed qualitatively the excitation of interlayer plasmons, which should occur at a temperature $\gtrsim T_{F}$, producing an enhancement to the drag resistivity. In Chapter 4 , we considered the effect of electron-phonon interaction on the quasiparticle properties. We derived an expression for the effective phonon-mediated electron-electron interaction and formulated the electron self-energy problem due to exchange of phonons. We then obtained exact analytical expressions for the imaginary and real parts of the self-energy, and calculated the quasiparticle spectral function. The imaginary part of the self-energy exhibits step jumps at the phonon energy characteristic of dispersionless phonons, and the real part of the self-energy shows logarithmic divergences 
at the phonon energy. We then calculated the renormalized quasiparticle velocity from the self-energy, finding it to exhibit a kink at the phonon energy due to the logarithmic divergence and theoretically explaining the observation in the ARPES experiment. Having considered how interaction effects renormalize the quasiparticle properties, we turned to the question how interaction effects renormalize phonon properties in Chapter 5. We first formulated the diagrams for the renormalization of the phonon Green function and the problem of phonon energy renormalization. We then formulated the problem of the phonon self-energy which we calculated in the long-wavelength limit and the static limit, before obtaining the exact result for general values of $q$ and $\omega$. We found interesting and novel Kohn anomalies from the phonon self-energy which are completely different from the Kohn anomaly in conventional metals. With the phonon self-energy, we then obtained the renormalized phonon energy dispersion. We found the Kohn anomalies in the phonon self-energy carry over to the phonon energy dispersion, and the phonon energy first increases and then decreases with respect to electron density. The presence of these novel Kohn anomalies - which are completely different from $q=2 k_{F}$ in conventional metals - are quite remarkable, originating from the dynamical nature and the chiral structure of the electron-phonon coupling in graphene.

We now discuss open problems as possible future projects that extend the work in this dissertation. The Coulomb drag problem in double-layer graphene can be extended to the case of a finite magnetic field (the so-called magnetodrag problem). Electron-hole layers can also be considered when bilayer exciton condensates can form. With the introduction of a dielectric material in between the 
two graphene layers, interlayer phonons will be present and phonon-mediated drag will be interesting to study. With electron-electron Coulomb interaction, it is also interesting to study the localization effect in the electrical conductivity from AronovAltshuler correction. Finally, the theory for the quasiparticle renormalization due to Coulomb interaction and electron-phonon interaction in Chapter 2 and 4, and the phonon renormalization in Chapter 5, can be generalized to include the case of bilayer graphene (where the two layers are $\sim \AA$ apart and strongly coupled). 


\section{List of Publications}

1. W.-K. Tse, B. Y.-K. Hu, and S. Das Sarma, "Chirality-induced Dynamic Kohn Anomalies in Graphene", arXiv:0801.1291.

2. S. Das Sarma, B. Y.-K. Hu, E.H. Hwang, and W.-K. Tse, "Electron-Electron Interactions in Graphene", arXiv:0708.3239.

3. W.-K. Tse and S. Das Sarma, "Phonon-Induced Many-body Renormalization of Graphene Electronic Properties", Phys. Rev. Lett. 99, 236802 (2007).

4. S.X. Zhang, W. Yu, S.B. Ogale, S.R. Shinde, D.C. Kundaliya, W.-K. Tse, S.Y. Young, J.S. Higgins, L.G. Salamanca-Riba, M. Herrera, L.F. Fu, N.D. Browning, R.L. Greene, and T. Venkatesan, "Magnetism and Anomalous Hall Effect in Co-(La,Sr)TiO3", Phys. Rev. B 76, 085323 (2007).

5. W.-K. Tse, B. Y.-K. Hu, and S. Das Sarma, "Theory of Coulomb Drag in Graphene", Phys. Rev. B 76, 081401(R) (2007).

6. S. Das Sarma, E. Hwang, and W.-K. Tse, "Many-Body Interaction Effects in Doped and Undoped Graphene: Fermi Liquid versus non-Fermi Liquid", Phys. Rev. B 75, 121406(R) (2007). 
7. W.-K. Tse and S. Das Sarma, "Coulomb Drag and Spin Coulomb Drag in the presence of Spin-orbit Coupling", Phys. Rev. B 75, 045333 (2007).

8. W.-K. Tse and S. Das Sarma, "Intrinsic Spin Hall Effect in the presence of Extrinsic Spin-Orbit Scattering", Phys. Rev. B 74, 245309 (2006).

9. W.-K. Tse and P.T. Leung, "Theory of Light Emission in Sonoluminescence as Thermal Radiation", Phys. Rev. E 73, 056302 (2006).

10. W.-K. Tse and S. Das Sarma, "Spin Hall Effect in Doped Semiconductor Structures", Phys. Rev. Lett. 96, 056601 (2006).

11. W.-K. Tse, J. Fabian, I. Zutic, and S. Das Sarma, "Spin Accumulation in the Extrinsic Spin Hall Effect”, Phys. Rev. B 72, 241303(R) (2005). 


\section{Bibliography}

[1] A.H. Castro Neto, F. Guinea, N.M.R. Peres, K.S. Novoselov, and A.K. Geim, arXiv:0709.1163 .

[2] A. Damascelli, Physica Scripta 61 (2004).

[3] S.Y. Zhou, G.-H. Gweon, and A. Lanzara, Ann. Phys. 321, 1730 (2006).

[4] A. Bostwick, T. Ohta, T. Seyller, K. Horn, and E. Rotenberg, Nature Phys. 3, $36(2007)$.

[5] A.C. Ferrari, J.C. Meyer, V. Scardaci, C. Casiraghi, M. Lazzeri, F. Mauri, S. Piscanec, D. Jiang, K.S. Novoselov, S. Roth, and A.K. Geim, Phys. Rev. Lett. 97, $187401(2006)$.

[6] E.H. Hwang and S. Das Sarma, Phys. Rev. B 75, 205418 (2007).

[7] K.S. Novoselov, A.K. Geim, S.V. Morozov, D. Jiang, M.I. Katsnelson, I.V. Grigorieva, S.V. Dubonos, and A.A. Firsov, Nature 438, 197 (2005).

[8] R.E. Peierls, Ann. I.H. Poincare 5, 177 (1935).

[9] L.D. Landau, Phys. Z. Sowjetunion 11, 26 (1937). 
[10] L.D. Landau and E.M. Lifshitz, Statistical Physics, Part 1 (Pergamon Press, Oxford, 1980).

[11] N.D. Mermin, Phys. Rev. 176, 250 (1968).

[12] A.K. Geim and K.S. Novoselov, Nature Mat. 6, 183 (2007).

[13] S. Das Sarma, A.K. Geim, P. Kim, and A.H. MacDonald, Solid State Commun. 143, (2007).

[14] T. Ando, J. Phys. Soc. Jpn. 74, 777 (2005).

[15] P.R. Wallace, Phys. Rev. 71, 622 (1947).

[16] J.W. McClure, Phys. Rev. 108, 612 (1957).

[17] J. Slonczewski and P. Weiss, Phys. Rev. 109, 272 (1958).

[18] J. Yan, Y. Zhang, P. Kim, and A. Pinczuk, Phys. Rev. Lett. 98, 166802 (2007).

[19] H. Suzuura and T. Ando, Phys. Rev. B 65, 235412 (2002).

[20] T. Ando, J. Phys. Soc. Jpn. 75, 124701 (2006).

[21] T. Valla, A.V. Fedorov, P.D. Johnson, and S.L. Hulbert, Phys. Rev. Lett. 83, 2085 (1999).

[22] A. Lanzara, P.V. Bogdanov, X.J. Zhou, S.A. Kellar, D.L. Feng, E.D. Lu, T. Yoshida, H. Eisaki, A. Fujimori, K. Kishio, J.-I. Shimoyama, T. Noda, S. Uchida, Z. Hussain, and Z.-X. Shen, Nature 412, 510 (2001). 
[23] S.Y. Zhou, G.-H. Gweon, J. Graf, A.V. Fedorov, C.D. Spataru, R.D. Diehl, Y. Kopelevich, D.-H. Lee, S. G. Louie, and A. Lanzara, Nature Phys. 2, 595 (2006).

[24] J.L. McChesney, A. Bostwick, T. Ohta, K.V. Emtsev, T. Seyller, K. Horn, and E. Rotenberg, arXiv:0705.3264v1 .

[25] S.Y. Zhou, G.-H. Gweon, A.V. Fedorov, P.N. First, W.A. de Heer, D.-H. Lee, F. Guinea, A.H. Castro Neto, and A. Lanzara, Nature Mat. 6, 770 (2007).

[26] S.Y. Zhou, D.A. Siegel, A.V. Fedorov, and A. Lanzara, arXiv:0801.3862v1 .

[27] S.Y. Zhou, D.A. Siegel, A.V. Fedorov, F. El Gabaly, A.K. Schmid, A.H. Castro Neto, and A. Lanzara, Nature Mat. 7, 259 (2008).

[28] S. Das Sarma, E.H. Hwang, and W.-K. Tse, Phys. Rev. B 75, 121406(R) (2007).

[29] W.-K. Tse, B. Y.-K. Hu, and S. Das Sarma, Phys. Rev. B 76, 081401(R) (2007).

[30] W.-K. Tse and S. Das Sarma, Phys. Rev. Lett. 99, 236802 (2007).

[31] W.-K. Tse, B. Y.-K. Hu, and S. Das Sarma, arXiv:0801.1291 .

[32] A.L. Fetter and J.D. Walecka, Quantum theory of many-particle systems (McGraw-Hill, New York, 1971).

[33] H. Bruus and K. Flensberg, Many-body quantum theory in condensed matter physics (Oxford University Press, New York, 2004). 
[34] G.D. Mahan, Many-particle Physics, 3rd ed. (Plenum Press, New York, 2000).

[35] K. W. K. Shung, Phys. Rev. B 34, 979 (1986).

[36] M. Polini, R. Asgari, Y. Barlas, T. Pereg-Barnea, and A.H. MacDonald, Phys. Rev. Lett. 98, 236601 (2007).

[37] B. Wunsch, T. Stauber, F. Sols, and F. Guinea, New J. Phys. 8, 318 (2006).

[38] L. Hedin and S. Lundqvist, Solid State Physics, (F. Seitz, D. Turnbull. and H. Ehrenreich, eds.) 23, 1 (1969).

[39] Y. Zhang and S. Das Sarma, Phys. Rev. B 71, 045332 (2005).

[40] A.V. Chaplik, JETP 33, 997 (1971).

[41] V.M. Galitski and S. Das Sarma, Phys. Rev. B 70, 035111 (2004).

[42] J.F. Janak, Phys. Rev. 178, 1416 (1969).

[43] J. González, F. Guinea, and M.A.H. Vozmediano, Phys. Rev. B 59, R2474 (1999).

[44] A.A. Abrikosov and S.D. Beneslavskii, JETP 32, 699 (1971).

[45] A.-P. Jauho and H. Smith, Phys. Rev. B 47, 4420 (1993).

[46] A. Kamenev and Y. Oreg, Phys. Rev. B 52, 7516 (1995).

[47] K. Flensberg, B. Y.-K. Hu, A.-P. Jauho, and J.M. Kinaret, Phys. Rev. B 52, $14761(1995)$. 
[48] T.J. Gramila, J.P. Eisenstein, A.H. MacDonald, L.N. Pfeiffer, and K.W. West, Phys. Rev. Lett. 66, 1216 (1991).

[49] W.-K. Tse and S. Das Sarma, Phys. Rev. B 75, 045333 (2007).

[50] E.H. Hwang, S. Das Sarma, V. Braude, and A. Stern, Phys. Rev. Lett. 90, $086801(2003)$

[51] K. Flensberg and B. Y.-K. Hu, Phys. Rev. Lett. 73, 3572 (1994).

[52] K. Flensberg and B. Y.-K. Hu, Phys. Rev. B 52, 14796 (1995).

[53] F. Tuinstra and J. Koenig, J. Chem. Phys. 53, 1126 (1970).

[54] L.M. Woods and G.D. Mahan, Phys. Rev. B 61, 10651 (2000).

[55] G.D. Mahan, Phys. Rev. B 68, 125409 (2003).

[56] K. Ishikawa and T. Ando, J. Phys. Soc. Jpn. 75, 084713 (2006).

[57] H. Suzuura and T. Ando, J. Phys. Soc. Jpn. 77, 044703 (2008).

[58] G. Grimval, The electron-phonon interaction in metals (North Holland, Amsterdam, 1981).

[59] S. Engelsberg and J.R. Schrieffer, Phys. Rev. 131, 993 (1963).

[60] S. Pisana, M. Lazzeri, C. Casiraghi, K.S. Novoselov, A.K. Geim, A.C. Ferrari, and F. Mauri, Nat. Mater. 6, 198 (2007). 
[61] A. Das, S. Pisana, S. Piscanec, B. Chakraborty, S.K. Saha, U.V. Waghmare, R. Yiang, H.R. Krishnamurhthy, A.K. Geim, A.C. Ferrari, and A.K. Sood1y, arXiv:0709.1174v1 .

[62] J. Yan, E.A. Henriksen, P. Kim, and A. Pinczuk, arXiv:0712.3879v1 .

[63] M. Lazzeri and F. Mauri, Phys. Rev. Lett. 97, 266407 (2006).

[64] W. Kohn, Phys. Rev. Lett. 2, 393 (1959).

[65] R. Nicklow, N. Wakabayashi, and H.G. Smith, Phys. Rev. B 5, 4951 (1972).

[66] J.L. Wilkes, R.E. Palmer, and R.F. Willis, J. Electron Spectrosc. Relat. Phenom. 44, 355 (1987).

[67] C. Oshima, T. Aizawa, R. Souda, Y. Ishizawa, and Y. Sumiyoshi, Solid State Commun. 65, 1601 (1988).

[68] S. Siebentritt, R. Pues, and K.-H. Rieder, Phys. Rev. B 55, 7927 (1997).

[69] C. Thomsen and S. Reich, Phys. Rev. Lett. 85, 5214 (2000).

[70] R. Saito, A. Jorio, A.G. Souza Filho, G. Dresselhaus, M.S. Dresselhaus, and M.A. Pimenta, Phys. Rev. Lett. 88, 027401 (2001).

[71] J. Maultzsch, S. Reich, C. Thomsen, H. Requardt, and P. Ordejón, Phys. Rev. Lett. 92, 075501 (2004).

[72] M. Mohr, J. Maultzsch, E. Dobardzic, S. Reich, I. Milosevic, M. Damnjanovic, A. Bosak, M. Krisch, and C. Thomsen, Phys. Rev. B 76, 035439 (2007). 
[73] S. Piscanec, M. Lazzeri, F. Mauri, A. Ferrari, and J. Robertson, Phys. Rev. Lett. 93, 185503 (2004).

[74] R. Jalabert and S. Das Sarma, Phys. Rev. B 40, 9723 (1989).

[75] A.H. Castro Neto and F. Guinea, Phys. Rev. B 75, 045404 (2007). 